\title{
Posterior Predictive Model Checks in Cognitive Diagnostic Models
}

\author{
Jung Yeon Park
}

Submitted in partial fulfillment of the requirements for the degree of Doctor of Philosophy under the Executive Committee of the Graduate School of Arts and Sciences

\section{COLUMBIA UNIVERSITY}


(C) 2015

Jung Yeon Park All Right Reserved 


\author{
ABSTRACT \\ Posterior Predictive Model Checks in Cognitive Diagnostic Models \\ Jung Yeon Park
}

Cognitive diagnostic models (CDMs; DiBello, Roussos, \& Stout, 2007) have received increasing attention in educational measurement for the purpose of diagnosing strengths and weaknesses of examinees' latent attributes. And yet, despite the current popularity of a number of diagnostic models, research seeking to assess model-data fit has been limited. The current study applied one of the Bayesian model checking methods, namely the posterior predictive model check method (PPMC; Rubin, 1984), to its investigation of model misfit. We employed the technique in order to assess the modeldata misfit from various diagnostic models, using real data and conducting two simulation studies. An important issue when it comes to the application of PPMC is choice of discrepancy measure. This study examines the performance of three discrepancy measures utilized to assess different aspects of model misfit: observed totalscores distribution, association of item pairs, and correlation between attribute pairs as adequate measures of the diagnostic models.

Keywords: posterior predictive model checking; discrepancy measures; cognitive diagnostic model; DINA; general diagnostic model 


\section{Table of Contents}

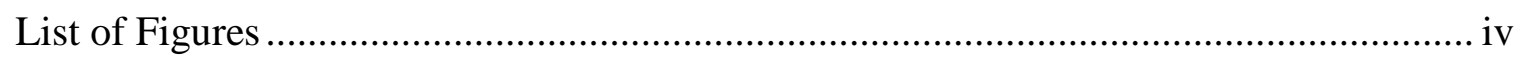

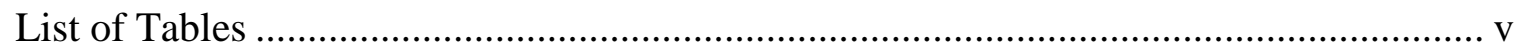

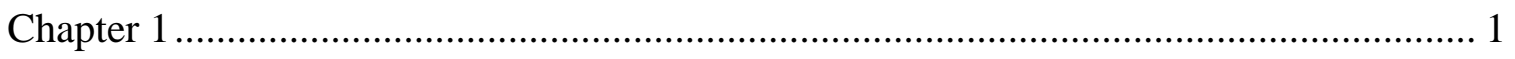

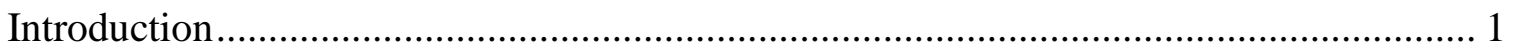

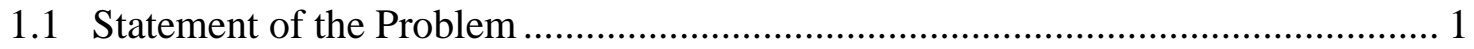

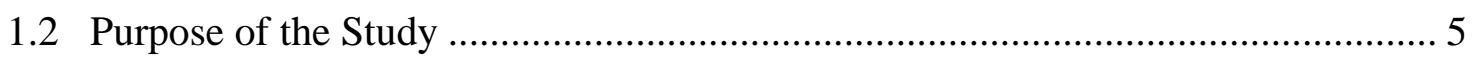

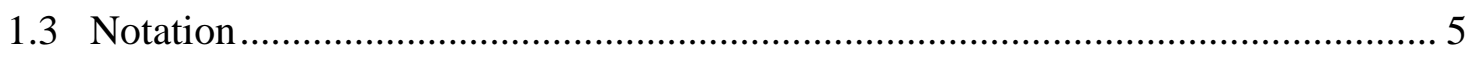

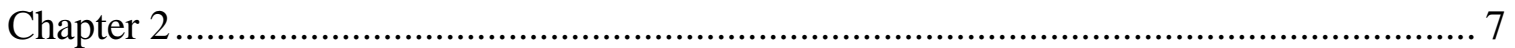

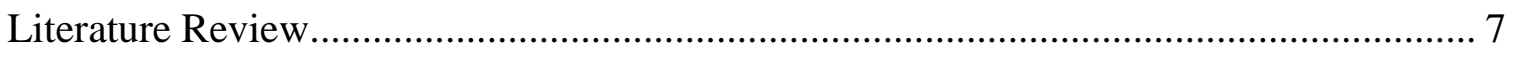

2.1 Item Response Theory Models.................................................................... 7

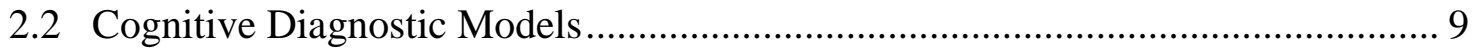

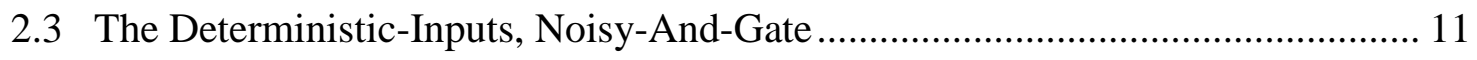

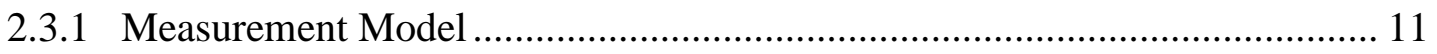

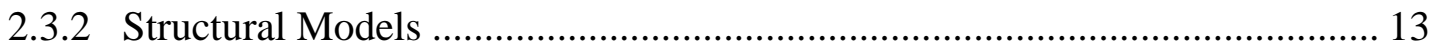

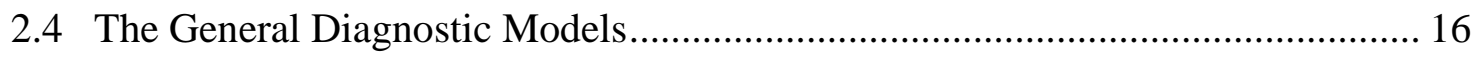

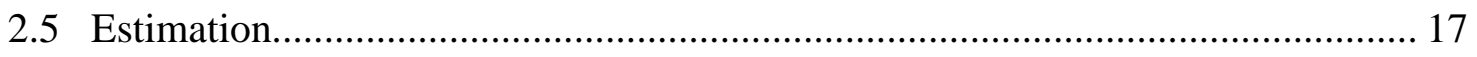

2.5.1 Marginal Maximum Likelihood Estimation (MMLE) ............................... 17

2.5.2 Bayesian Inference with Markov Chain Monte Carlo (MCMC)................... 18

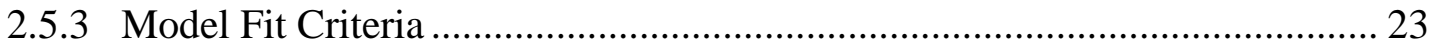

2.6 Posterior Predictive Model Checks .................................................................. 23

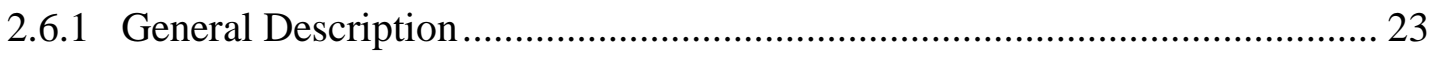

2.6.2 PPMC for Latent Variable Models .......................................................... 27

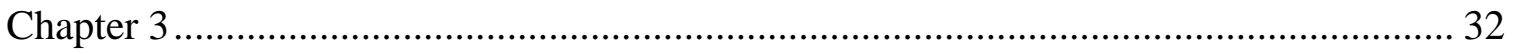

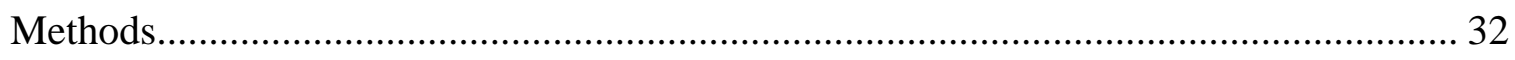

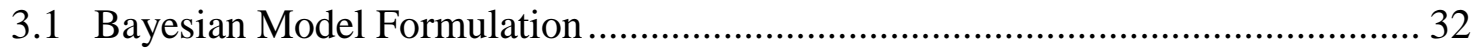

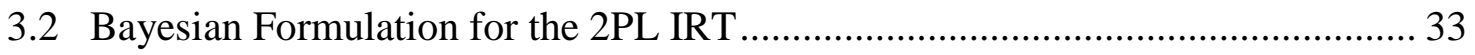


3.3 Bayesian Formulation for the DINAs .............................................................. 34

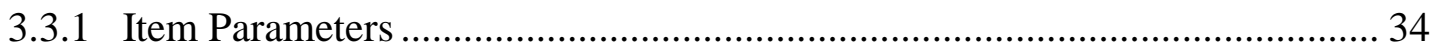

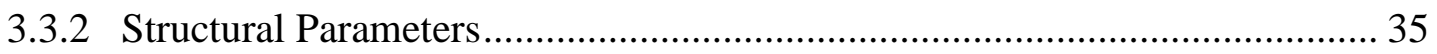

3.4 Bayesian Formulations for the GDMs ............................................................. 38

3.4.1 Item Parameters ................................................................................... 38

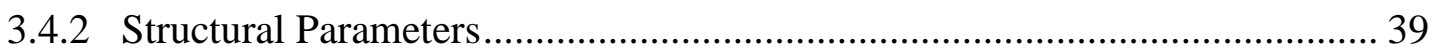

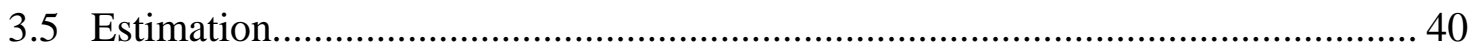

3.5.1 MCMC algorithms using BUGS ................................................................ 40

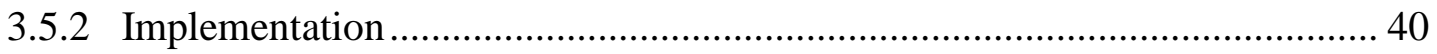

3.5.3 Model Diagnostic Criteria ………………………........................................ 41

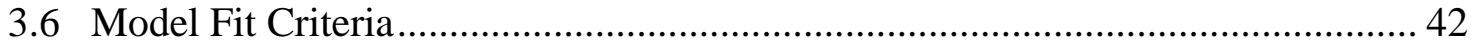

3.6.1 Generating Data from Posterior Predictive Distribution ................................. 42

3.6.2 Proposed Discrepancy Measures …………………………………………... 43

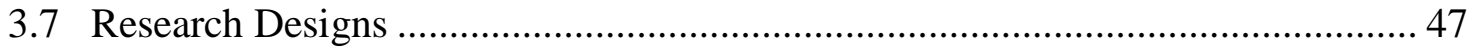

3.7.1 Empirical Data Example........................................................................... 47

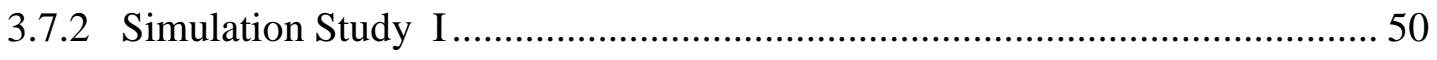

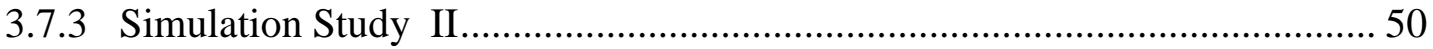

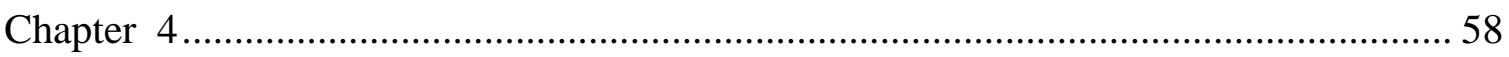

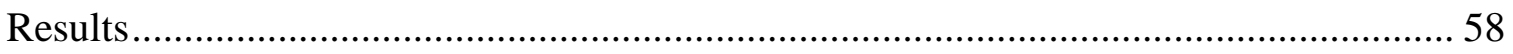

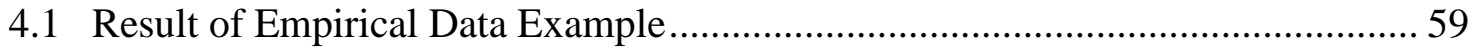

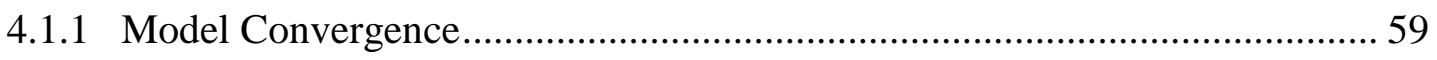

4.1.2 Total Score Distribution .............................................................................. 59

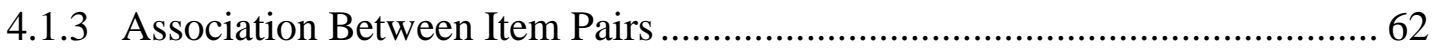

4.1.4 Association Between Attribute Pairs ................................................................ 68

4.2 Result of Simulation Study I ........................................................................... 70

4.2.1 Total Score Distribution ............................................................................ 70

4.2.2 Association Between Item Pairs ................................................................... 72

4.2.3 Association Between Attribute Pairs ............................................................... 75

4.3 Result of Simulation Results II ………………............................................. 77 


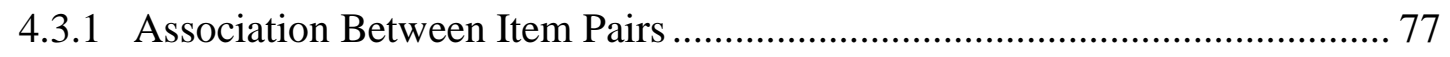

4.3.2 Association Between Attribute Pairs ........................................................ 81

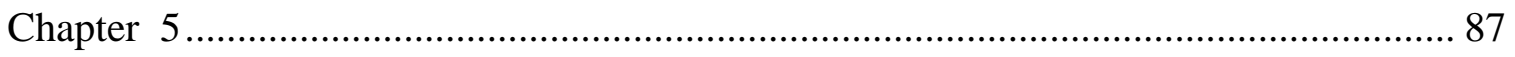

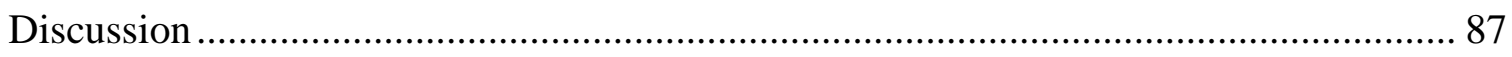

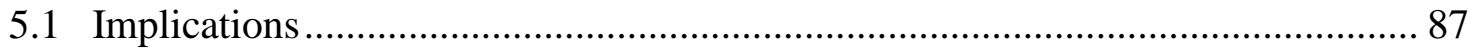

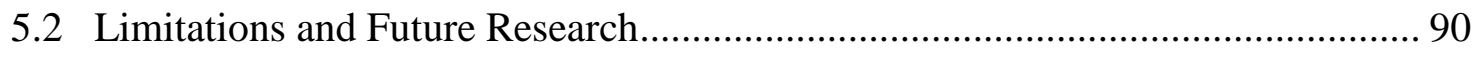

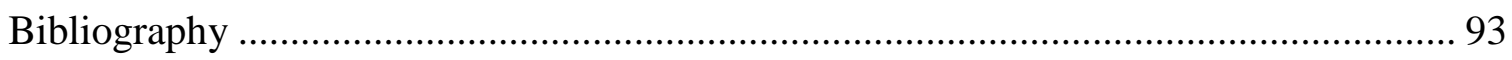

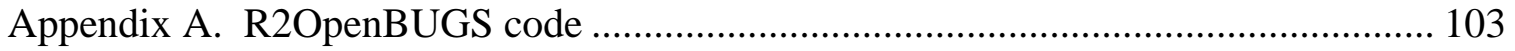

Appendix B. R code for Posterior Predictive Model Checks ..................................... 107

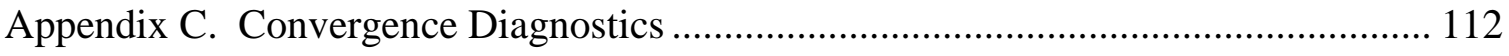




\section{List of Figures}

Figure 2.1 Summary of Posterior Predictive Model Checks (PPMC) .25

Figure 3.1 Joint Probability Density Function of Anti-slip Parameter

and Guessing Parameter...

Figure 3.2 Example to Demonstrate Utility of Item-Pair Odds Ratio and Attribute-Pair

Correlation ...... 46

Figure 3.3 Distribution of Total Score (Fraction Subtraction Data)

Figure 4.1 Summary of the Posterior Predictive Distributions of the Observed Total

Scores (Fraction Subtraction Data)

Figure 4.2 Posterior Predictive P-values for Item-pair Odds Ratios (Fraction Subtraction

Data). .66

Figure 4.3 Summary of the Posterior Predictive Distributions of the Observed Total Scores (Simulation Study I)

Figure 4.4 Posterior Predictive P-values for Item-pair Odds Ratios (Simulation Study I).

Figure 4.5 Posterior Predictive P-values from the Pearson Product-Moment Correlation between Per Skill Sum Scores (Simulation Study I) . .76

Figure 4.6 Summary of Extreme P-values from Item-Pair Odds Ratio and Pearson Product-Moment Correlations between Per-Skill Sum Scores (Simulation study II) . .85 


\section{List of Tables}

Table 3.1 Q-matrix Designed for Fraction Subtraction Data ......................................48

Table 3.2 Q-matrix Designed for Simulation Study II...........................................53

Table 4.1 Posterior Predictive P-values from the Pearson Product-Moment Correlation between Per Skill Sum Scores (Fraction Subtraction Data) ......................................69

Table 4.2 Percentage of Extreme P-values for Item-Pair Odds Ratios (Simulation Study

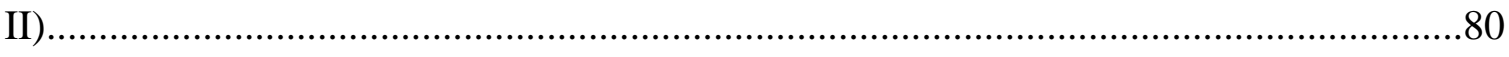

Table 4.3 Posterior Predictive P-values from the Pearson Product-Moment Correlation

between Per-Skill Sum Scores (Simulation Study II) ............................................. 84 


\section{Acknowledgement}

First and foremost, I would like to express my sincere gratitude to my advisor, Professor Matthew S. Johnson and my mentor, Professor Young-Sun Lee for their generous support, patience, and understanding. I appreciate the time and effort they spent on my studies and my professional life. I am deeply indebted to them for guiding various research projects, lectures, dissertation, and graduate study in general. Their scholarship, commitment to students, and generosity have inspired me in my research and teaching. I would like to express my deep appreciation to Professor Melanie M. Wall, for supporting my scholarly visions. I am deeply indebted to her for the intensive amount of guidance and generosity. I cannot imagine the last six years without the three professors; it has been such a great learning experience with them.

Also I would like to thank my committee members, Professor James E. Corter and Professor Ryan S. Baker for their time, constructive comments, and suggestions for this dissertation. Also, I would like to express my deep appreciation to all my great teachers from Columbia University, George Washington University, and Ewha Womans University.

In addition, I could not have completed my study without the support and encouragement of my fellow graduate students, Dr. Jianzhou Zhang, Ms. Chenmu Xing, Dr. Sultana Mustafa, Mr. Bright Nsowaa, Mr. Huacheng Li, Ms. Nayeon Yoo, and many others. We not only helped with each other with our work but also shared so many laughs 
and smiles in GD 556. Also, I thank Dr. Sunghee Shin and Dr. Jane Yoo for their encouragement.

Last but not least, I would not be here and have reached this far without the support of my father, Dr. Chang Shik Park. His endless care and support let me pursue my doctoral degree worry free because I know he will back my decisions completely and will always be there for me when I need him the most. I would like to dedicate this dissertation to my mother, Ms. Gui Soon Lee. I always remember her endless love and prayers; I hope you are proud of me from heaven. I also thank my brother, Dr. Daniel Park, sister-in-law, Ms. SoJung Kim, and lovely niece SeoJin Park. I also thank Mr. Bijit Roy, my "best" friend; my journey in the U.S. wouldn't be possible without your support and confidence. Thank you.

Finally, I thank my God for allowing many wonderful things in my life. I am always grateful to make my dreams come true. 


\section{Chapter 1}

\section{Introduction}

\subsection{Statement of the Problem}

Cognitive diagnostic models (CDMs; DiBello, Roussos, \& Stout, 2007) are a type of latent class model in which each item in the assessment measures a small number of discrete cognitive skills or attributes. Most CDMs treat the attributes as categorical latent variables; typically they are binary variables, indicating whether examinees have mastered or failed to master the skills or attributes. Thus, the purpose of the models is to diagnose and categorize each examinee according to a fine-grained attribute-profile or pattern of skills they possess. Over the last decade, CDMs have received increasing attention from researchers of educational measurement and statistics, because of both their extensive diagnostic potential and the richer information that modeling multiple latent attributes can provide. The major advantage of CDMs over traditional IRT (item response theory) framework is that they provide information about the specific skills or attributes that an examinee has or has not mastered as revealed by an educational achievement test (Henson, Templin, and Willse, 2009). Thus CDMs are more useful for attribute-assessment than are traditional IRT models, for the latter estimate a broader construct of ability in just one dimension. In other words, CDMs are capable of providing more precise statistical evidence that measure why a person has failed an exam. 
One of the key components that many CDMs share is a capability to specify the relationship between items and attributes. This relationship, also called the Q-matrix (Tatsuoka, 1985), is defined by a matrix of $J$ rows and $K$ columns where the rows represent items and the columns represent attributes. Typically, every element $\left(q_{j k}\right)$ in the Q-matrix is dichotomous; $q_{j k}=1$ indicates that the item $j$ requires attribute $k$. Similarly, $q_{j k}=0$ indicates that the item $J$ does not require attribute $K$. In general, the Qmatrix is constructed by subject-matter experts before data are collected or models are fitted.

In CDMs, attributes typically are coded in a binary fashion (0/1), identifying each examinee as exhibiting the attribute mastery or non-mastery. If there are $K$ attributes required for examinee $i$ in a test, then it is denoted as a vector of binary values, $\boldsymbol{\alpha}_{i}=$ $\left(\alpha_{i 1}, \ldots, \alpha_{i K}\right)^{T}$, which results in a total of $2^{K}$ possible patterns of the latent classes. Thus if $K=2$, there are four possible patterns: $(0,0),(0,1),(1,0)$, or $(1,1)$.

There is a wide range of models that fall within the framework of CDMs. The models often are characterized by the rules for how the attributes combine, and are of either the conjunctive or the compensatory type (Rupp, Templin, and Henson, 2010). The "conjunctive" models assume that the lack of a required attribute cannot be compensated for by other attributes; examples are the DINA (Deterministic-Input, Noisy-And-Gate) model of Junker and Sijtsma (2001), the NIDA (Noisy-Input, Deterministic-And-Gate) model of Junker and Sijtsma (2001), and the NC-RUM (Non-Compensatory Reparameterized Unified Model) model of Hartz (2002). By contrast, "compensatory" models assume that the lack of an attribute can be compensated for by another attribute 
required for the item; examples are the DINO (Deterministic-Input, Noisy-Or-Gate) model of Templin and Henson (2006), the NIDO (Noisy-Input, Deterministic-Or-Gate) model of Templin (2006), and the compensatory GDM (General Diagnostic Model) of von Davier (2005).

The DINA model (Junker and Sijtsma, 2001; Haertel, 1989; Macready and Dayton, 1977) is one of the conjunctive (or non-compensatory) CDMs most often used by researchers in educational measurements. The model assumes that a correct response on an item depends on possessing all the attributes required to solve that item. To reduce the complexity of estimation in a fully unstructured attribute space, various attribute models have been suggested; examples include the independence model (Maris, 1999) and the higher-order model (HO-DINA; de la Torre and Douglas, 2004). On the other hand, the DINO model (Templin and Henson, 2006) is deemed a compensatory analog to the DINA model (Rupp et al., 2010). The model assumes that a correct response on an item depends on possessing at least one of the required attributes for the item.

As compared to the two models mentioned above, the GDMs (von Davier, 2005) are a more general type of diagnostic model, one that may be parameterized to reflect either compensatory or conjunctive rules. The compensatory rule inherent in the GDMs allows a subset of all the required attributes to individually contribute to a correct response for the item; thus if an examinee has mastered more of the required attributes for the item, the probability of a correct response on the item increases.

Despite the current popularity of the CDMs, research on assessing model-data fit is considerably limited. This is problematic, because checking model fit is crucial to 
evaluating the strengths and weaknesses of the proposed models. The model fit criteria to diagnose the current model helps to determine how one should improve the model. Even if the model would not be modified or replaced, one should be aware of its limitations to make inferences. Indeed the issue of the model diagnostics provides the motivation for this study, where we focus on model diagnostic measures for the DINA model and the GDM while using a Bayesian approach. Specifically, we use posterior predictive model checks (PPMC) as our tool for assessing of model fit.

Posterior predictive model checking (PPMC; Rubin, 1984; Guttman, 1967; Gelman, Meng, and Stern, 1996; Meng, 1994) is a popular Bayesian model checking tool. It has a close connection to the frequentist goodness-of-fit tests (Gelman, Meng, and Stern, 1996) and can be thought of as its Bayesian equivalent. The method compares the observed data with replicated data generated from the posterior predictive distribution using discrepancy measures. A discrepancy measure is an analogue of a classical test statistic. It measures the difference between some aspect of the observed data and the replicated data sets. Typically we generate a large number of data sets from the posterior predictive distribution, i.e. the distribution of replicated data conditional on the observed data, and compare using the discrepancy measures. Systematic differences between the observed data set and the replicated data sets are captured by the discrepancy measures and indicate potential failures of the model to explain the data.

Although the PPMC has been recognized as a promising technique for evaluating psychometric models such as unidimensional IRT models (Sinharay, Johnson, and Stern, 2006), multidimensional IRT models (Levy, Mislevy, and Sinharay, 2009; Levy, 2011), 
and Bayesian networks (Sinharay, 2006), the methodology has been relatively unexplored within the framework of CDMs. Hence the importance of addressing the issue in this dissertation.

\subsection{Purpose of the Study}

In this study, we monitor the varying performances of different PPMC methods in detecting model-data misfit based on various diagnostic models while using a real data example and two simulation studies. An important factor when it comes to the application of PPMC is the choice of model fit indices or discrepancy measures. This study examines the performance of three discrepancy measures for assessing different aspects of model fit: (a) observed total-score distribution, (b) association of item pairs, and (c) correlation of attribute pairs. The diagnostic models include DINA models with three attribute models including the (a) independence model, (b) higher-order model, and (c) unstructured model, plus GDM models with the assumption of (a) constant slopes and (b) varying slopes. We also compared the models with the two-parameter logistic (2PL) IRT model.

\subsection{Notation}

The following is a list of the basic notation and specification used in this dissertation.

Item Responses Let $Y_{i j}$ denote the binary response of individual $i$ to item $j$, where 1 denotes correct answers. Then $\mathbf{Y}$ represents a $N \times J$ matrix of $N$ individuals and $J$ items in total, where $Y_{i j} \in\{0,1\}, i=1, \ldots, N$, and $j=1, \ldots, J$, as below: 


$$
\mathbf{Y}=\left[\begin{array}{ccc}
Y_{11} & \cdots & Y_{N 1} \\
\vdots & \ddots & \vdots \\
Y_{1 J} & \cdots & Y_{N J}
\end{array}\right]
$$

Q-matrix The Q-matrix is a matrix of ones and zeroes, where $q_{j k}$ denotes a binary indicator of item $j$ and attribute $k$, where $q_{j k}=1$ indicates item $j$ requires skill or attribute $k$, and where $j=1, \ldots, J$, and $k=1, \ldots, K$, as below:

$$
\mathbf{Q}=\left[\begin{array}{ccc}
q_{11} & \cdots & q_{J 1} \\
\vdots & \ddots & \vdots \\
q_{1 K} & \cdots & q_{J K}
\end{array}\right]
$$

Attribute Parameter Let $\alpha_{i k}$ denote an indicator if an individual $i$ has mastered skill $k$ or has failed to do so. Then $\boldsymbol{\alpha}_{i}=\left(\alpha_{i 1}, \ldots, \alpha_{i K}\right)^{\boldsymbol{T}}$ denotes a vector to represent an attribute pattern of examinee $i$ where $\alpha_{i k} \in\{0,1\}, j=1, \ldots, J$, and $k=$ $1, \ldots, K$. In other words, $\alpha_{i k}=1$ indicates that examinee $i$ has mastered attribute $k$. Similarly, $\alpha_{i k}=0$ indicates that examinee $i$ failed to master attribute $k$. 


\section{Chapter 2}

\section{Literature Review}

This chapter provides with some vital background knowledge on the key concepts of this study. We begin, in section 2.1 and 2.2 , by formulating more general concepts in the item response theory models and the cognitive diagnostic models. Section 2.3 provides an overview of DINA (Deterministic, Inputs, Noisy "And" Gate; Junker and Sijtsma, 2001) model, one of the most popular CDMs over the last decade. This section includes a formulation of the measurement model and makes several structural assumptions about the attribute space. Section 2.4 provides an overview of general diagnostic models (GDMs; von Davier, 2005).

Next, in section 2.5, we review two parameter-estimation methods, Maximum Likelihood Estimation (MLE) and Bayesian Estimation with MCMC algorithms. Finally, in section 2.6, we talk about Bayesian model checking methods, posterior predictive model checks (PPMC). In the same section, we review the discrepancy measures used to assess various aspects of the model fit of the various latent variable models.

\subsection{Item Response Theory Models}

Item response theory (IRT) models are latent variable models that relate a set of categorical observed variables to a set of continuous latent variable(s). In this section, we 
conduct an overview of the characteristics of unidimensional IRT models having binary responses: (a) the Rasch model (Rasch, 1960) and (b) the two-parameter logistic model (2PL; Birnbaum,1969). For ease of explanation, we start with the 2PL model before the Rasch model. Let $Y_{i}=\left(Y_{i 1}, \ldots, Y_{i J}\right)$ denote a vector of response pattern for the examinee $i$, where $Y_{i j} \in\{0,1\} ; i=1, \ldots, N$ (examinee); $j=1, \ldots, J$ (item).

The 2PL assumes that the probability of endorsing item $j$ can be characterized by a subject-level latent trait (or "ability") parameter, an item-level location (or "difficulty") parameter, and an item-level slope (or "discrimination") parameter. Let $\xi$ be the latent trait, $v_{1 j}$ be the location, and $v_{0 j}$ be the slope parameter. Using a logit link function, the item response function (IRF) for the model is:

$$
\begin{gathered}
\operatorname{Logit} P_{i j}=v_{0 j}\left(\xi-v_{1 j}\right) \\
\text { i.e. } P_{i j}=P\left(Y_{i j}=1 \mid v_{0 j}, v_{1 j}, \xi\right)=\frac{e^{v_{0 j}\left(\xi-v_{1 j}\right)}}{1+e^{v_{0 j}\left(\xi-v_{1 j}\right)}} .
\end{gathered}
$$

The item difficulty $\left(v_{1 j}\right)$ is the level of latent trait $(\xi)$ associated with a 50 percent chance of endorsing the item $j$. The item discrimination, $\left(v_{0 j}\right)$, is the slope of the IRF at the item difficulty. This indicates how much information item $j$ provides to discriminate among examinees according to their latent traits.

The Rasch model (Rasch, 1960) assumes that the probability of endorsing the item $j$ is characterized by a subject-level latent trait parameter and an item-level location parameter. Using a logit link function, the IRF for the Rasch model is:

$$
\text { Logit } P_{i j}=\operatorname{Logit}\left(Y_{i j}=1 \mid v_{1 j}, \xi\right)=\xi-v_{1 j}
$$


As another formulation, the slope parameter $\left(v_{0}\right)$ can be included as a scaling factor that is constant across all items, i.e. $v_{0}=v_{01}=\cdots=v_{0 J}$ :

$$
\text { Logit } P_{i j}=\operatorname{Logit}\left(Y_{i j}=1 \mid v_{0}, v_{1 j}, \xi\right)=v_{0}\left(\xi-v_{1 j}\right)
$$

Assuming conditional independence among $J$ items given $\xi$, the general formulation of the likelihood function for the IRT models is as follows:

$$
L\left(\boldsymbol{v}_{0}, \boldsymbol{v}_{1}, \xi\right)=\prod_{i=1}^{N} \prod_{j=1}^{J} P_{i j}^{Y_{i j}}\left[1-P_{i j}\right]^{1-Y_{i j}}
$$

where $i=1, \ldots, N$ (examinee); $j=1, \ldots, J$ (item).

\subsection{Cognitive Diagnostic Models}

The cognitive diagnostic models (CDMs) are latent variable models that relate a set of categorical observed variables to a set of categorical latent variables. In this section, we conduct an overview of the general characteristics of the CDMs where the observed responses and the latent variable are binary. In CDMs, the probability of endorsing item $j$ is modeled by using two components: a subject-level attribute parameter and two itemlevel parameters, namely "guessing" and "slip" parameters. The subject-level attribute parameter $\alpha_{i k}$ indicates whether the examinee $i$ has mastered or failed to master the attribute $k$ :

$$
\alpha_{i k}=\left\{\begin{array}{cc}
1, & \text { if masters attribute } k \\
0, & \text { otherwise }
\end{array}\right.
$$

where $k=1, \ldots, K$ (attribute). In CDMs, the $\alpha_{i k}$ is then combined with the Q-matrix (Tatsuoka, 1983) to determine if the attribute $k$ is required to deal with the item $j$. The components of the Q-matrix are as follows: 


$$
q_{j k}=\left\{\begin{array}{cc}
1, & \text { if attribute } k \text { is required for item } j \\
0, & \text { otherwise }
\end{array}\right.
$$

As shown above, $q_{j k}=1$ represents that the item $j$ requires the attribute $k$ for, $j=$ $1, \ldots, J$, and $k=1, \ldots, K$, and vice versa. Typically the Q-matrix is determined by subject experts beforehand, so it is treated as a fixed quantity, as if the true associations of attributes versus items were already known. Recently, a few studies (e.g. Liu, Xu, and Ying, 2012; Xiang, 2013) have been done on data-driven approaches to identification of the Q-matrix and estimation of related model parameters. They are beyond the scope of this dissertation, however, and thus will not be covered.

The functional form of an individual attribute $\left(\alpha_{i k}\right)$ and each component of the Qmatrix $\left(q_{j k}\right)$ is as follows:

$$
\eta_{i j}=f\left(\alpha_{i k}, q_{j k}\right)
$$

where $f($.$) is some function. The \eta_{i j}$ in equation (2.7), "ideal response" (Tatsuoka, 1990, 1985) or "latent response" (Maris, 1995), indicates whether the examinee should answer item $j$ correctly given the examinee's attribute pattern, i.e., $\boldsymbol{\alpha}=\left(\alpha_{1}, \ldots, \alpha_{K}\right)^{T}$. How one defines the latent response is the key to differentiating various CDMs. In general, there are two extreme ways of defining the latent response function, or $\eta_{i j}$; that is, "compensatory" models in which different attributes combine, so that a deficit in one attribute may be made up for by another attribute, and "non-compensatory" or "conjunctive" models in which the lack of one attribute cannot be compensated for by another attribute. 
In a deterministic situation, the latent responses coincide with observed responses; $Y_{i j}=\eta_{i j}$. Most CDMs, however, assume that there are uncertainties in the relationship noted above, meaning that the observed responses do not exactly represent latent responses. For the reason, many CDMs incorporate item-level parameters (e.g. guessing and slip). The item-level parameters are discussed in more detail in following sections.

\subsection{The Deterministic-Inputs, Noisy-And-Gate}

\subsubsection{Measurement Model}

In this section, we provide the item response function (IRF) for the DeterministicInputs, Noisy-And-Gate (or "DINA") model. Let $Y_{i j}$ be the random response of examinee $i$ to item $j$, where $i=1, \ldots, N ; j=1, \ldots, J$. Then, $Y_{i j}=1$ if the examinee $i$ answers the item $j$ correctly or $Y_{i j}=0$, otherwise. Also, let us denote the examinee's latent attribute by $\alpha_{i k}$, where $k=1, \ldots, K ; \alpha_{i k}=1$ indicates if an examinee has mastered attribute $k$ or $\alpha_{i k}=0$, otherwise. Thus the attribute pattern of examinee $i$ can be denoted by $\boldsymbol{\alpha}_{i}=$ $\left(\alpha_{i 1}, \ldots, \alpha_{i K}\right)^{T}$. In order to implement the CDMs, we need to determine whether the attribute $k$ is necessary in order to answer item $j$. The Q-matrix (Tatsuoka, 1985) is a binary matrix, where, $q_{j k}=1$ indicates that attribute $k$ is required to solve item $j$ correctly, and $q_{j k}=0$, otherwise.

The DINA model (Junker and Sijtsma, 2001; Haertel, 1989; Macready and Dayton, 1977) typically is characterized by a latent response 


$$
\eta_{i j}=\prod_{k=1}^{K} \alpha_{i k}^{q_{j k}}
$$

where $\boldsymbol{\alpha}_{i}=\left(\alpha_{i 1}, \ldots, \alpha_{i K}\right)^{T}$ is a vector of knowledge-states and $\boldsymbol{\eta}=\left(\eta_{1}, \ldots, \eta_{J}\right)^{T}$ is a vector of latent responses to reflect the deterministic prediction of task performance based on the attribute pattern of the examinee. The latent response definition implies that one should master all of the required attributes if one wishes to have a correct ideal response.

The latent-response pattern $\boldsymbol{\eta}$ is linked to observed responses in a probabilistic relationship with two "noisy" parameters, the slipping $\left(s_{j}\right)$ and guessing $\left(g_{j}\right)$ parameters. The guessing rate is the probability that examinee $i$ responds correctly to item $j$ even though s/he does not possess all the required attributes:

$$
g_{j}=P\left(Y_{i j}=1 \mid \eta_{j}=0\right)
$$

The slip rate is the probability that examinee $i$ fails to respond to item $j$ correctly even though s/he possesses all the required attributes:

$$
s_{j}=P\left(Y_{i j}=0 \mid \eta_{j}=1\right) \text {. }
$$

Therefore, the item response function (IRF) is written as

$$
P\left(Y_{i j}=1 \mid \boldsymbol{\alpha}\right)=\left(1-s_{j}\right)^{\eta_{i j}} g_{j}^{1-\eta_{i j}}
$$

The local independence assumption leads to $P\left(\boldsymbol{Y}_{i} \mid \boldsymbol{\alpha}\right)=\prod_{j=1}^{J} P\left(\boldsymbol{Y}_{i j} \mid \boldsymbol{\alpha}\right)$; the $Y_{i j} \mathrm{~s}$ are independent for all $j$ s, given the attribute pattern $\boldsymbol{\alpha}$.

Typically, there is a constraint on the two item parameters; the "anti-slip" rate $\left(=1-s_{j}\right)$ is always higher than the "guessing" rate. In particular, the item response function is monotone in the coordinates of $\alpha_{i}$, as long as $1-s_{j}>g_{j}$. In order to ensure 
this relationship, Junker and Sijtma (2001) explored whether the monotonicity actually holds within real data. They used BUGS program to approximate the posterior distributions of the parameters of the DINA model using DEDSTRAT data; their Bayesian model assumed flat prior distributions of the guessing and slip parameters, i.e,.

$g_{j} \sim U\left(0, g_{\max }\right)$ and $s_{j} \sim U\left(0, s_{\max }\right)$. The results suggested that most of the point estimates satisfied monotonicity, i.e., $1-s_{j}>g_{j}$, or equivalently $s_{j}+g_{j}<1$.

Assuming conditional independence among $J$ items given $\boldsymbol{\alpha}$, the likelihood function for the DINA model with binary responses is:

$$
L(\boldsymbol{Y})=\prod_{i=1}^{N} \prod_{j=1}^{J}\left[s_{j}^{1-y_{i j}}\left(1-s_{j}\right)^{y_{i j}}\right]^{\eta_{i j}}\left[g_{j}^{y_{i j}}\left(1-g_{j}\right)^{1-y_{i j}}\right]^{1-\eta_{i j}}
$$

where $\eta_{i j}=\prod_{k=1}^{K} \alpha_{i k}^{q_{j k}}$

\subsubsection{Structural Models}

a. Independence Structure The CDMs can be viewed as a type of multiple classification latent class model (MCLCM; Maris, 1999), in the sense that the models assume that each examinee is characterized by his or her membership in one of multiple latent classes, each of which belongs to one latent classification. In Maris (1999), the author explained the possible structures of the latent class memberships within the MCLCM. One of the proposed models for $\alpha_{k}$ is to assume that the $\alpha_{k} \mathrm{~s}$ are independently distributed (Maris, 1999). Because $\alpha_{k}$ is binary, it is characterized by $K$ parameters $\pi_{k}$ which are equal to $P\left(\alpha_{k}=1\right)$. Then,

$$
P(\boldsymbol{\alpha}=1)=\prod_{k=1}^{K} \pi_{k} .
$$


Hence, the probability mass function (pmf) is

$$
P(\boldsymbol{\alpha})=\prod_{k=1}^{K} \pi_{k}^{\alpha_{k}}\left(1-\pi_{k}\right)^{1-\alpha_{k}} .
$$

b. Higher-order Structure The purpose of the higher-order DINA model is to model the joint distribution of a vector of attributes using the structure of higher-order latent traits. The model is motivated by the need for a relatively simple formulation of the joint distribution in settings where the notion of higher-order latent traits represents a construct of general knowledge defined more broadly than are the attributes in the cognitive diagnostic model (de la Torre and Douglas, 2004).

The higher-order model assumes conditional independence—specifically, that the attributes are conditionally independent under a higher-order ability parameter, $\theta$, which is defined by

$$
P(\boldsymbol{\alpha}=1 \mid \theta)=\prod_{k=1}^{K} P\left(\alpha_{k}=1 \mid \theta\right),
$$

where $P\left(\alpha_{k}=1 \mid \theta\right)=\frac{\exp \left(b_{k}+a_{k} \theta\right)}{\left[1+\exp \left(b_{k}+a_{k} \theta\right)\right]}$.

Here $b_{k}$ indicates the attribute-level location parameter and $a_{k}$ indicates the attributelevel slope parameter. By denoting $\pi_{k}=P\left(\alpha_{k}=1 \mid \theta\right)$, this model can be characterized by a pdf:

$$
P(\boldsymbol{\alpha} \mid \theta)=\prod_{k=1}^{K} \pi_{k}^{\alpha_{k}}\left(1-\pi_{k}\right)^{1-\alpha_{k}}
$$

c. Saturated Structure Let us recall that $\boldsymbol{\alpha}=\left(\alpha_{1}, \ldots, \alpha_{K}\right)^{T}$ denotes a vector of attribute mastery indicators, where $\alpha_{i k} \in\{0,1\}, k=1, \ldots, K$. This model assumes that the probabilities of each of the different realizations of $\alpha_{k}$ are unrestricted, allowing for all possible statistical dependences of any possible pairwise relationship between $\alpha_{i k}$ and 
$\alpha_{i k}$, where $k=k^{\prime}$. Because the $\alpha_{k}$ is binary, the total number of parameters for attribute patterns in $\boldsymbol{\alpha}$ is $2^{K}-1$.

Then the probability that the $K$-dimensional vector $\boldsymbol{\alpha}$ takes on $l$-th pattern is characterized by a single parameter $\pi_{l}$.

The model is described by the probability mass function (pmf)

$$
P(\boldsymbol{\alpha})=\prod_{l=1}^{2^{K}} \pi_{l}^{I_{l}(\boldsymbol{\alpha})},
$$

where $I_{l}$ is an indicator function having the value 1 if $\boldsymbol{\alpha}$ is the $l$-th pattern, and 0 otherwise.

There are a few shortcomings to the saturated model. Firstly, the unstructured attribute distribution is computationally expensive because the total number of latent classes increases dramatically as the number of attributes increase. For example, when there are $K=3$ attributes in a DINA model, $7\left(=2^{3}-1\right)$ latent class parameters need to be estimated. If $K=9$ attributes, however, then $511\left(=2^{9}-1\right)$ latent class parameters need to be estimated. Whereas information contained within the $2^{K}-1$ parameters can provide meaningful insights into the structure and nature of the attributes in the form of whether or not attribute hierarchies are present, typically it is insufficient to summarize and characterize the distribution of attributes relying upon these parameters alone (Rupp et al., 2010). Secondly, more informative and parsimonious ways to summarize associations among the attributes can be done by fitting models to the attribute structures (e.g. higher-order model). 


\subsection{The General Diagnostic Models}

The general class of GDMs (von Davier, 2005; von Davier \& Yamamoto, 2004a 2004b) was developed with the goal of maintaining similarities to previous approaches that are based on IRT, log-linear models, and latent class analysis but also incorporating the Q-matrix to the model. One of the advantages of the GDM lies in its capability to apply attribute models to polytomous item responses and to endow attributes with more than two proficiency levels.

Let us assume that both response outcome and attribute proficiency are dichotomous i.e. $\alpha_{k} \in\{0,1\}$ and $Y_{i j} \in\{0,1\}$. If we assume a logit link function, the model can be formulated as

$$
\begin{aligned}
& \text { Logit } P_{i j}=\operatorname{Logit} P\left(Y_{j}=1 \mid \beta_{j}, \gamma_{j}, \boldsymbol{\alpha}\right)=\beta_{j}+\gamma_{j}^{T} h\left(\boldsymbol{q}_{j}, \boldsymbol{\alpha}\right), \\
& \text { i.e } P_{i j}=P\left(Y_{i j}=1 \mid \beta_{j}, \gamma_{j}, \boldsymbol{\alpha}\right)=\frac{\exp \left[\beta_{j}+\gamma_{j}^{T} h\left(\boldsymbol{q}_{j}, \boldsymbol{\alpha}\right)\right]}{1+\exp \left[\beta_{j}+\gamma_{j}^{T} h\left(\boldsymbol{q}_{j}, \boldsymbol{\alpha}\right)\right]},
\end{aligned}
$$

with $K$ attributes (discrete latent traits), $\boldsymbol{\alpha}=\left(\alpha_{1}, \ldots, \alpha_{K}\right)^{T}$ as the attribute proficiency and $\boldsymbol{q}_{j}$ as the set of attributes influencing item $j$ as given by the $j$-th row of the Q-matrix. The equation shows that the probability of a correct response can be seen as incorporating two parts: the overall difficulty, i.e., $\beta_{j}$, and a linear combination of interactions of attributes required and attributes present: $h\left(\boldsymbol{q}_{j}, \boldsymbol{\alpha}\right)=\left(h_{1}\left(\boldsymbol{q}_{j}, \boldsymbol{\alpha}\right), \ldots, h_{m}\left(\boldsymbol{q}_{j}, \boldsymbol{\alpha}\right)\right)$.

In this study, we use $h\left(\boldsymbol{q}_{j}, \boldsymbol{\alpha}\right)=\left(q_{j 1} \alpha_{1}, \ldots, q_{j K} \alpha_{K}\right)$. Assuming that there is a nonzero Q-matrix entry and that the slope parameters $(\gamma)$ may be different for each attribute, the slope parameter $\gamma_{j k}$ determines the degree to which the particular attribute components in $\boldsymbol{\alpha}=\left(\alpha_{1}, \ldots, \alpha_{K}\right)^{T}$ contribute to the response probabilities for item $j$ : 


$$
\begin{aligned}
\operatorname{Logit} P\left(Y_{j}\right. & \left.=1 \mid \beta_{j}, \gamma_{j}, \boldsymbol{\alpha}\right)=\beta_{j}+\sum_{k=1}^{K} \gamma_{k j} q_{k j} \alpha_{k} \\
& =\beta_{j}+\gamma_{1 j} q_{1 j} \alpha_{1}+\cdots+\gamma_{K j} q_{K j} \alpha_{K} .
\end{aligned}
$$

Similarly, under the constant slopes assumption, $h\left(\boldsymbol{q}_{j}, \boldsymbol{\alpha}\right)=\sum_{k=1}^{K} q_{k j} \alpha_{k}$. In this case the probability of correct response is influenced only by the number of required attributes present. This model also can be thought of as a constant-slope simplification of the previous GDM (i.e., all the $\gamma_{j k}$ 's are equal wherein $k=1, \ldots, K$ ). That is,

$$
\begin{aligned}
\operatorname{Logit} P\left(Y_{j}\right. & \left.=1 \mid \beta_{j}, \gamma_{j}, \boldsymbol{\alpha}\right)=\beta_{j}+\gamma_{j} \sum_{k=1}^{K} q_{k j} \alpha_{k} \\
& =\beta_{j}+\gamma_{j}\left(q_{1 j} \alpha_{1}+\cdots+q_{K j} \alpha_{K}\right) .
\end{aligned}
$$

Assuming conditional independence among $J$ items given $\boldsymbol{\alpha}$, the likelihood function for the GDMs with binary responses is seen to be

$$
L(\boldsymbol{\beta}, \boldsymbol{\gamma} ; \boldsymbol{\alpha})=\prod_{i=1}^{N} \prod_{j=1}^{J} P_{i j}{ }^{Y_{i j}}\left[1-P_{i j}\right]^{1-Y_{i j}},
$$

where $P_{i j}=P\left(Y_{i j}=1 \mid \beta_{j}, \gamma_{j}, \boldsymbol{\alpha}\right)$.

\subsection{Estimation}

In this section, we discuss two main methods of obtaining estimates for the parameters in CDMs. The first method is Marginal Maximum Likelihood Estimation (MMLE) and the second method is Bayesian Estimation.

\subsubsection{Marginal Maximum Likelihood Estimation (MMLE)}

In parameter estimation using the classical MLE method, the joint maximum likelihood estimation (JMLE) is one of the possible maximum likelihood estimation 
procedures. The joint maximum likelihood estimation procedure treats both item parameters and propensities as unknown and fixed model parameters (Johnson, 2007). Thus, this procedure estimates the item-level parameter (i.e., $s_{j}$ and $g_{j}$ ) and the subjectlevel parameter (i.e., $\boldsymbol{\alpha}$ ) in CDMs by maximizing the likelihoods with respect to all parameters simultaneously.

This method has barely been used for CDMs, however. It certainly should be used with caution in the DINA model (de la Torre, 2009) because joint maximization of the subject-level parameter and the item-level parameters may lead to inconsistent estimators within a traditional IRT framework (see Baker, 1992; Neyman \& Scott, 1948). That fact tends to debunk JMLE as a reliable method in a CDMs framework.

As an alternative method, the marginal maximum likelihood estimation (MMLE) procedure has been widely used. This procedure assumes that the subject-level parameter $(\boldsymbol{\alpha})$ is a random effect sampled from some larger population, and integrates the parameter out of likelihood (Johnson and Junker, 2003; Johnson, 2007).

Thus, the likelihood function is maximized with respect to the item-level parameters, $\boldsymbol{g}$ and $\boldsymbol{s}$. That is,

$$
P(\boldsymbol{Y} \mid \boldsymbol{s}, \boldsymbol{g})=\prod_{i=1}^{N}\left[\sum_{l=1}^{2^{K}} \pi_{\boldsymbol{\alpha}_{l}} \prod_{j=1}^{J} P\left(Y_{i j}=y_{i j} \mid s_{j}, g_{j}, \boldsymbol{\alpha}\right)\right]
$$

where $\left(Y_{i j}=1 \mid s_{j}, g_{j}, \boldsymbol{\alpha}\right)=\left(1-s_{j}\right)^{\eta_{i j}} g_{j}{ }^{1-\eta_{i j}}$ and $\pi_{\boldsymbol{\alpha}_{l}}$ is the prior probability of $\boldsymbol{\alpha}_{l}$.

\subsubsection{Bayesian Inference with Markov Chain Monte Carlo (MCMC)}


Bayesian inference draws conclusions about the parameters using probability distributions of the parameters, given observed data. It combines prior knowledge about the parameters and the likelihood of the data to arrive at a posterior distribution of the model parameters.

Let us denote the prior distribution of the parameters by $P(\boldsymbol{\Psi})$ and the likelihood function of the model by $L(\boldsymbol{Y}, \boldsymbol{\Psi})$. Then the posterior distribution of $\boldsymbol{\Psi}, P(\boldsymbol{\Psi} \mid \mathbf{Y})$, is proportional to the product of $P(\boldsymbol{\Psi})$ and $L(\mathbf{Y}, \boldsymbol{\Psi})$ :

$$
\begin{aligned}
P(\boldsymbol{\Psi} \mid \mathbf{Y}) & =\frac{L(\mathbf{Y}, \boldsymbol{\Psi}) P(\boldsymbol{\Psi})}{\int L(\mathbf{Y}, \boldsymbol{\Psi}) P(\boldsymbol{\Psi}) d \boldsymbol{\Psi}} \\
& \propto L(\mathbf{Y}, \boldsymbol{\Psi}) P(\boldsymbol{\Psi}),
\end{aligned}
$$

where $\boldsymbol{\psi}$ is a set of parameters used in the model.

Often it is not feasible to deal with the posterior distribution $P(\Psi \mid \mathbf{Y})$ analytically, in which case Markov chain Monte Carlo (MCMC) techniques typically are employed. Monte Carlo method generally refers to any type of simulation technique in which sufficiently large numbers of points are randomly sampled out of a probability distribution of interest, and then are summarized in order to approximate particular characteristics of the distribution.

A Markov chain is a type of stochastic process in which the probability of current states of the process relies only on its immediate past. Therefore, each of the sampled values is dependent only on its preceding value. When it is not possible to sample directly from the target distribution we construct a Markov chain such that, once this chain has run for sufficiently long, it will converge to the target distribution. 
The basic principle behind the MCMC algorithms is that any quantity of interest about the posterior distribution can be empirically estimated by setting up a suitable Markov chain, running it long enough (burn-in), and using the subsequent values (Gill, 2007). Various techniques are used to implement the MCMC.

a. Metropolis-Hastings Algorithm The Metropolis-Hastings algorithm (Metropolis, Rosenbluth, Rosenbluth, Teller, and Teller, 1953; Hastings, 1970) is a type of acceptance-rejection simulation technique (Chib and Greenberg, 1995). Essentially, the acceptance-rejection algorithm seeks to generate samples from a candidate (or "proposal") distribution from which samples can easily be generated, and then keeping or rejecting the sample based on some criteria. The resulting samples behave as if they were drawn from the target distribution.

Let us suppose we want to sample the parameter $\boldsymbol{\psi}$ drawn from a target distribution, $p(\boldsymbol{\Psi})$. First we select a proposal distribution having the same support as the target distribution. At the $t$-th step of the Markov chain, when the chain is at the position $\boldsymbol{\Psi}^{[t-1]}$, we draw $\boldsymbol{\Psi}^{*}$ from the proposal distribution, $q\left(\boldsymbol{\Psi}^{*}, \boldsymbol{\Psi}^{[t-1]}\right)$. In order to determine the transition from the current state (i.e. $\boldsymbol{\Psi}^{[t-1]}$ ) to the future state (i.e. $\boldsymbol{\Psi}^{[t]}$ ), an acceptance ratio is needed to compare the target density at the candidate value with that at the current state. In the original Metropolis algorithm (Metropolis et al., 1953), the acceptance ratio was suggested as follow:

$$
r=\frac{p\left(\Psi^{*}\right)}{p\left(\Psi^{[t-1]}\right)}
$$


Later, for the purpose of increasing the speed of convergence, Hastings (1970) provided this modification of the ratio:

$$
r=\frac{p\left(\boldsymbol{\Psi}^{*}\right) / q\left(\boldsymbol{\Psi}^{*}, \boldsymbol{\Psi}^{[t-1]}\right)}{p\left(\boldsymbol{\Psi}^{[t-1]}\right) / q\left(\boldsymbol{\Psi}^{[t-1]}, \boldsymbol{\Psi}^{*}\right)},
$$

and we introduce a probability $\alpha_{M H}$ such that $\alpha_{M H}=\min [r, 1]$. If the $\alpha_{M H}$ is greater than or equal to a random sample drawn from $\mathrm{U}(0,1)$, then $\boldsymbol{\Psi}^{*}$ becomes $\boldsymbol{\Psi}^{[t]}$; otherwise, we start over with a new sample $\boldsymbol{\Psi}^{*}$ drawn from the proposal distribution.

b. Gibbs Sampling When parameter space is a multi-dimensional, sampling from the joint posterior distribution typically is difficult. The Gibbs sampler (Geman and Geman, 1984) partitions the multi-dimensional parameters into sub-blocks (or scalars), constructs conditional distributions of the blocks, and then sequentially samples from the conditional distributions of the sub-blocks.

Suppose we want to sample the $D$-dimensional parameter, $\boldsymbol{\Psi}$, drawn from a target distribution, $p(\boldsymbol{\Psi})$. We calculate $\left(\boldsymbol{\Psi}_{1} \mid \boldsymbol{\Psi}_{2}, \boldsymbol{\Psi}_{3}, \ldots, \boldsymbol{\Psi}_{D}\right), \ldots, p\left(\boldsymbol{\Psi}_{D} \mid \boldsymbol{\Psi}_{1}, \boldsymbol{\Psi}_{2}, \ldots, \boldsymbol{\Psi}_{D-1}\right)$. These are called the full-conditional distributions. Then the Gibbs sampler works as follows, with $T$ iterations.

For $t=1$ to $T$,

Sample $\boldsymbol{\Psi}_{1}^{[t]}$ from $p\left(\boldsymbol{\Psi}_{1} \mid \boldsymbol{\Psi}_{2}^{[t-1]}, \boldsymbol{\Psi}_{3}^{[t-1]}, \ldots, \boldsymbol{\Psi}_{D}^{[t-1]}\right)$

Sample $\boldsymbol{\Psi}_{2}^{[t]}$ from $p\left(\boldsymbol{\Psi}_{2} \mid \boldsymbol{\Psi}_{1}^{[t]}, \boldsymbol{\Psi}_{3}^{[t-1]}, \ldots, \boldsymbol{\Psi}_{D}^{[t-1]}\right)$

Sample $\boldsymbol{\Psi}_{D}^{[t]}$ from $p\left(\boldsymbol{\Psi}_{D} \mid \boldsymbol{\Psi}_{1}^{[t]}, \boldsymbol{\Psi}_{2}^{[t]}, \ldots, \boldsymbol{\Psi}_{D-1}^{[t]}\right)$

After the $D$ sampling steps, we get 


$$
\boldsymbol{\Psi}^{[t]}=\left(\boldsymbol{\Psi}_{1}^{[t]}, \boldsymbol{\Psi}_{2}^{[t]}, \ldots, \boldsymbol{\Psi}_{D}^{[t]}\right)
$$

c. Metropolis within Gibbs Often it is convenient to combine the Metropolis-Hastings algorithm and Gibbs Sampling to establish a stationary target distribution. The hybrid algorithm known as the "Metropolis within Gibbs algorithm" (Tierney, 1994; Patz and Junker, 1999) sequentially samples from the full conditionals according to the Gibbs algorithm but introduces the $\mathrm{M}-\mathrm{H}$ algorithm when a fully conditional distribution is not a closed form or is difficult to obtain.

Patz and Junker (1999) have described as follows how the Metropolis-withinGibbs algorithm works in 2PL IRT model. Recall from section 2.1 that the item response function for the 2PL IRT is:

$$
\operatorname{Logit} P_{i j}=\operatorname{Logit}\left(Y_{i j}=1 \mid v_{0 j}, v_{1 j}, \xi\right)=v_{0 j}\left(\xi-v_{1 j}\right),
$$

where $v_{0 j}$ indicates an item discrimination for item $j, v_{1 j}$ indicates an item difficulty for item $j$, and $\xi$ indicates a subject ability parameter.

$\boldsymbol{a}$. In order to draw $\xi^{[t]}$ from the full conditional distribution at the $t$-th iteration, first draw a candidate point, $\xi^{*}$ drawn from a proposal distribution, $q\left(\xi^{*} ; \xi^{[t-1]}\right)$, and accept with the rate

$$
\alpha_{M H}=\min \left\{1, \frac{L\left(\xi^{*}, v^{[t-1]}\right) p\left(\xi^{*}, v^{[t-1]}\right) q\left(\xi^{*} ; \xi^{[t-1]}\right)}{L\left(\xi^{[t-1]}, v^{[t-1]}\right) p\left(\xi^{[t-1]}, v^{[t-1]}\right) q\left(\xi^{[t-1]} ; \xi^{*}\right)}\right\}
$$

b. In order to draw $v^{[t]}$ from the full conditional distribution at the $t$-th iteration, first draw a candidate point, $v^{*}$ from a proposal distribution, $q\left(v^{*} ; v^{[t-1]}\right)$, and accept with the rate 


$$
\alpha_{M H}=\min \left\{1, \frac{L\left(v^{*}, \xi^{[t]}\right) p\left(v^{*}, \xi^{[t]}\right) q\left(v^{*} ; v^{[t-1]}\right)}{L\left(v^{[t-1], \xi^{[t]}}\right) p\left(v^{[t-1]}, \xi^{[t]}\right) q\left(v^{[t-1]} ; v^{*}\right)}\right\}
$$

\subsubsection{Model Fit Criteria}

Many studies have used relative model fit criteria to demonstrate the usefulness of their proposed model. Typically various criteria for measuring global model fits are used. The global fit includes information criteria, i.e., AIC (Akaike's Information Criterion; Akaike, 1974); BIC (Bayesian Information Criterion; Schwarz, 1976); and DIC (Deviance Information Criterion; Spiegelhalter et al., 2002). Many studies of CDMs have used AIC and BIC to present relative model fit (DeCarlo, 2011; de la Torre \& Douglas, 2004). Tseng (2010) used DIC to compare DINA, LC ("Linear Compensatory"), and QUIC (“Quasi Compensatory”) models. The Bayes factor also was used to compare a DINA model with an assumption of attribute independence to the HO-DINA model (de la Torre \& Douglas, 2004).

\subsection{Posterior Predictive Model Checks}

\subsubsection{General Description}

The general idea behind posterior predictive model checks (PPMC) is that if the model fits the data properly, then one may expect that data replicated or generated from the model should look similar to the observed data. In other words, the observed data should look plausible under its posterior predictive distribution. The basic methodology for checking model fit is to draw simulated responses from the posterior predictive 
distribution, or the posterior distribution of replicated data, and then compare the values to the observed one. Basically, the distribution of replicated data is obtained under the assumption that the current state of knowledge is truth. Thus, any systematic differences between the simulated data and the observed data indicate that there is a potential misfit for the model.

a. Posterior Predictive Distribution Let $L(\mathbf{Y}, \boldsymbol{\Psi})$ denote a likelihood function, where $\boldsymbol{\Psi}$ indicates a set of model parameters, let $P(\boldsymbol{\Psi})$ be a prior distribution, and let $P(\boldsymbol{\Psi} \mid \mathbf{Y})$ denote a posterior distribution of on the parameters. Then a prior predictive distribution is defined as a distribution of replicated data before the full data were observed. That is,

$$
P\left(\mathbf{Y}^{\text {rep }}\right)=\int P\left(\mathbf{Y}^{\text {rep }}, \boldsymbol{\psi}\right) d \boldsymbol{\psi}=\int L\left(\mathbf{Y}^{\text {rep }}, \boldsymbol{\Psi}\right) P(\boldsymbol{\Psi}) d \boldsymbol{\Psi}
$$

Similarly, a posterior predictive distribution is defined as a distribution of replicated data after full data have been observed. That is,

$$
\begin{aligned}
P\left(\mathbf{Y}^{\text {rep }} \mid \boldsymbol{Y}\right) & =\int P\left(\mathbf{Y}^{\text {rep }}, \boldsymbol{\Psi} \mid \boldsymbol{Y}\right) d \boldsymbol{\Psi} \\
& =\int \frac{P\left(\mathbf{Y}^{\text {rep }}, \boldsymbol{\Psi} \mid \boldsymbol{Y}\right)}{P(\boldsymbol{\Psi} \mid \boldsymbol{Y})} P(\boldsymbol{\Psi} \mid \boldsymbol{Y}) d \boldsymbol{\Psi} \\
& =\int P\left(\mathbf{Y}^{\mathrm{rep}} \mid \mathbf{Y}, \boldsymbol{\Psi}\right) P(\boldsymbol{\Psi} \mid \mathbf{Y}) d \boldsymbol{\Psi} .
\end{aligned}
$$

As shown in equation (2.35), the posterior predictive distribution is viewed as a combination of a likelihood probability of the current model of interest fitted to replicated data and a posterior probability of the parameter of interests, $\boldsymbol{\Psi}$. Because $\mathbf{Y}^{\mathrm{rep}}$ and $\mathbf{Y}$ are conditionally independent given the $\boldsymbol{\Psi}, P\left(\mathbf{Y}^{\mathrm{rep}} \mid \mathbf{Y}, \boldsymbol{\Psi}\right)$ can be simplified as $P\left(\mathbf{Y}^{\mathrm{rep}} \mid \boldsymbol{\Psi}\right)$. Therefore, 


$$
P\left(\mathbf{Y}^{\text {rep }} \mid \mathbf{Y}\right)=\int L\left(\mathbf{Y}^{\text {rep }}, \boldsymbol{\Psi}\right) P(\boldsymbol{\psi} \mid \mathbf{Y}) d \boldsymbol{\psi} .
$$

The posterior predictive distribution of the replicated data will be a reference distribution for the observed $\mathbf{Y}$.

Figure 2.1 Summary of Posterior Predictive Model Checks (PPMC)

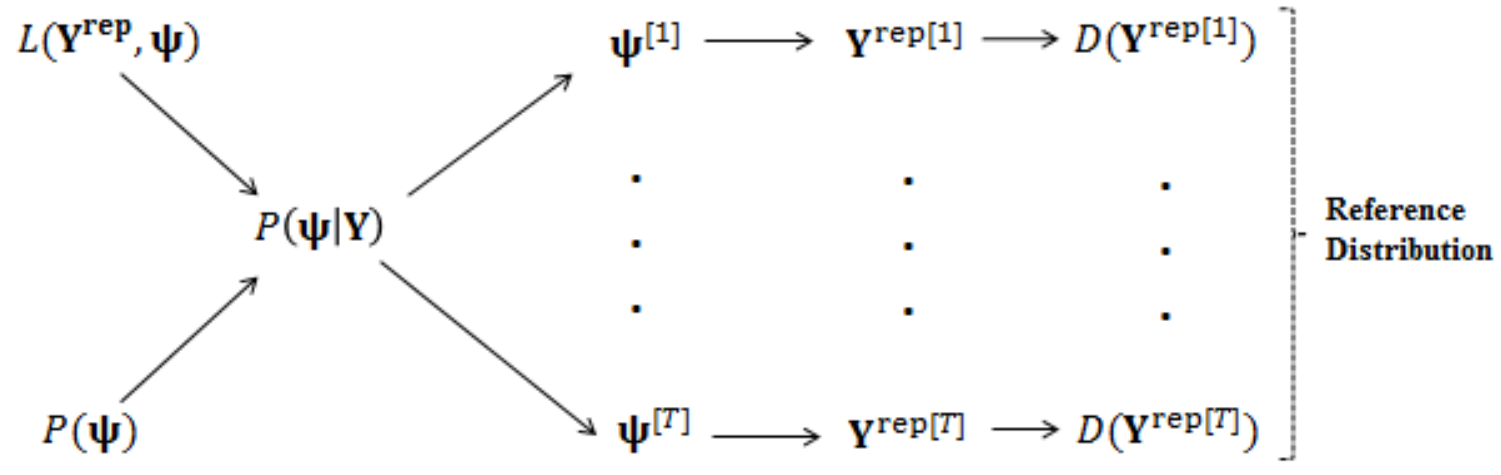

b. Discrepancy Measures The discrepancy between a fitted model and observed data can be measured by using types of summarizing quantities similar to the test statistics employed in classical testing approaches. These quantities, called "discrepancy measures" can vary depending on which aspect of the model we wish to check. A test summary or discrepancy measure $D(\mathbf{Y}, \mathbf{\Psi})$ is a scalar summary of parameters and data that is used as a standard when one is comparing data to predictive simulations. Whereas in a classical approach the test statistic is computed by using observed data only, in the Bayesian context the test statistics are dependent on the model parameters under their posterior distribution. A researcher may then use $D(\mathbf{Y}, \mathbf{\Psi})=D(\mathbf{Y})$, as a discrepancy measure depending on the data only. In which case, the PPMC consists 
of comparing $D(\mathbf{Y})$ to a posterior predictive distribution of $D\left(\mathbf{Y}^{\text {rep }}\right)$ (Sinharay, Johnson, and Stern, 2006).

c. Posterior Predictive P-value The amount of discrepancy between observed data and replicated data from the posterior predictive distribution can be measured by a tail-area probability which is computed by using posterior simulations of $\left(\mathbf{Y}^{\mathrm{rep}}, \boldsymbol{\Psi}\right)$. A model is suspect if a discrepancy is of practical importance and its observed value has a tail-area probability that is close to 0 or 1 , thereby indicating that the observed pattern would be unlikely to be seen in replication of the data if the model were true. The Bayesian counterpart of the classical $p$-value (Bayesian $p$-value) is defined by

$$
p \text {-value }=P\left[D\left(\mathbf{Y}^{\text {rep }}\right) \geq D(\mathbf{Y}) \mid \mathbf{Y}\right] .
$$

An extreme $p$-value implies that the model cannot replicate that aspect of the observed data. A major failure of the model, typically corresponding to extreme tail-area probabilities, can be addressed by expanding the model in an appropriate way. A lesser failure might also suggest the need for model improvements or might be ignored in the short term if the failure appears not to affect the main inferences. Sometimes it is possible to find the closed form or the posterior predictive distribution, $P\left(\mathbf{Y}^{\mathrm{rep}} \mid \mathbf{Y}\right)$, but often it is easier to sample from $P\left(\mathbf{Y}^{\text {rep }} \mid \mathbf{Y}\right)$ using Monte Carlo methods.

Rubin (1984) suggests simulating replicated data sets from the posterior predictive distribution in practical applications of the PPMC method. That is, one draws $T$ simulations $\boldsymbol{\Psi}^{[1]}, \ldots, \boldsymbol{\Psi}^{[T]}$ from the posterior distribution $P(\boldsymbol{\Psi} \mid \mathbf{Y})$ of $\boldsymbol{\Psi}$, and draws $\mathbf{Y}^{\mathrm{rep}[1]}, \ldots, \mathbf{Y}^{\mathrm{rep}[\mathrm{T}]}$ using the likelihood $L\left(\mathbf{Y}^{\mathrm{rep}}, \boldsymbol{\Psi}\right)$. The process results in $T$ draws from the posterior predictive $P\left(\mathbf{Y}^{\text {rep }} \mid \mathbf{Y}\right)$. The estimates of posterior predictive $p$-value are 
computed by calculating the proportion of replicated data in which $D\left(\mathbf{Y}^{\mathrm{rep}[\mathrm{t}]}\right)$ is greater than $D(\mathbf{Y})$. Similar to any two-tailed hypothesis testing, with a significance level at $\alpha$, if the proportion (posterior predictive $p$-value) is less than equal to $\alpha / 2$ or greater than equal to $1-\alpha / 2$, the proportion is considered as the extreme $\mathrm{p}$-value

According to Robins, van der Vaart, and Ventura (2000), depending on the choice of discrepancy measures, the posterior predictive $p$-value can sometime be so conservative that it often fails to detect model misfit. Wang, Shu, Shang, and Xu (2015) showed that a chi-square goodness-of-fit test statistic, using the PPMC method, tends to have a lower detection rate for item-level fit than the statistic using the EM algorithm.

Sinharay (2006) commented that such a conservative test with a reasonable power rate would be acceptable in educational testing, because evidences of model-data misfit lead to the discarding of the test items from the item pool by the test administrators.

\subsubsection{PPMC for Latent Variable Models}

The choice of discrepancy measures should reflect substantive aspects of the theory of interest and features of the data that may not have been adequately modeled (Levy, Mislevy, and Sinharay, 2009). This section explores the univariate and bivariate discrepancy measures suggested for (a) uni- and multi- dimensional item response theory models, (b) Bayesian networks, and (c) cognitive diagnostic models. 
a. Proportion Correct The "proportion correct" is a univariate measure used to determine whether the model can predict the distribution of sum-scores. For the binary response of item $j$, the measure is defined by

$$
P C_{j}=\frac{\sum_{i=1}^{N} Y_{i j}}{N},
$$

where $Y_{i j} \in\{0,1\} ; i=1, \ldots, N$ (examinee); $j=1, \ldots, J$ (item).

b. Latent Class Membership Two item-level $\chi^{2}$ types of measures were proposed based on latent class membership and raw score membership for applications of Bayesian network (Sinharay, 2006) and CDMs (Sinharay and Almond, 2007). For each item $j(j=1, \ldots, J)$ and each latent class membership $l(l=1, \ldots, L)$, let $\hat{p}_{l j}$ be a proportion of examinees who belong to latent class membership $l$ and answered item $j$ correctly. Note that the $\hat{p}_{l j}$ is calculated by the median proportion of the $\hat{p}_{l j}$ 's across all outputs of the MCMC iterations. $N_{l}$ is obtained by the mean value of the number of examinees in each latent class membership $l$ across all outputs of the MCMC iterations. Then the corresponding observed number of examinee is equal to $O_{j l}=\hat{p}_{l j} N_{l}$. Similarly, the $P_{l j}$ is obtained by the posterior mean of the proportion of examinees who belong to latent class membership $l$ and who answered item $j$ correctly, and thus the expected number of examinees is $E_{j l}=P_{l j} N_{l}$. Then the item fit statistic is defined by

$$
D_{j}^{\chi}=\sum_{l=1}^{L} N_{l} \frac{\left(O_{j l}-E_{j l}\right)^{2}}{E_{j l}\left(N_{l}-E_{j l}\right)} .
$$

The $G^{2}$-type measure based on the latent class membership is given by

$$
D_{j}^{G}=2 \sum_{l=1}^{L}\left[O_{j k} \log \left(\frac{O_{j l}}{E_{j l}}\right)+\left(N_{l}-O_{j l}\right) \log \left(\frac{N_{l}-E_{j l}}{N_{l}-E_{j l}}\right)\right] .
$$


Finally, an overall discrepancy measure for assessing model misfit can be obtained by summing all the items. Similarly, those two measures can be used based on raw score instead of attribute pattern $l$. The raw score is defined as the proportion of individuals in raw score group $r=1, \ldots,(R-1)$ who answer item $r$ correctly.

Alagoz (2012) applied the two proposed measures $D_{j}^{\chi}$ and $D_{r}^{\chi}$ to the DINA model having independence and higher-order structures. The study found that $D_{j}^{\chi}$ rejected all misspecified items and never rejected correctly specified items for both independent DINA and higher-order DINA; the Type 1 error was almost zero and power was close to 1. It was found, however that $D_{r}^{\chi}$ identified some correctly specified items as problematic. As a limitation of this measure, the author mentioned that it did not produce an acceptable Type I error rate and power, which could imply that $D_{r}^{\chi}$ is not sufficient when it comes to estimating mastery status on attributes with the two models.

c. Odds Ratio The odds ratio is a measure of association that is not dependent on the marginal distribution of the observables (Liebetrau, 1983). Sinharay and Johnson (2006) and Sinharay (2005) applied the posterior predictive model check to find the odds ratios corresponding to the responses to pairs of items discrepancy measures useful in detecting misfit of the unidimensional IRT models. The authors also found the odds ratio to be a useful discrepancy measure for detecting data-model misfit in several types of model misspecifications caused by local dependencies among the items. Let $n_{s s^{\prime}}$ denote the number of individuals scoring $s$ on the first item and $s^{\prime}$ on the second item where $s \in\{0,1\}$ and $s^{\prime} \in\{0,1\}$. Then 


$$
O R=\frac{n_{11} n_{00}}{n_{10} n_{01}}
$$

Levy (2011) proposed the log-transformed odds ratios as a discrepancy measure by taking the natural log scale.

$$
\ln \left(O R_{j j^{\prime}}\right)=\ln \left[\frac{\left(n_{11}\right)\left(n_{00}\right)}{\left(n_{10}\right)\left(n_{01}\right)}\right]=\ln \left(n_{11}\right)+\ln \left(n_{00}\right)-\ln \left(n_{10}\right)-\ln \left(n_{01}\right)
$$

Also, in order to check the assumption of the local dependence among items, a standardized log odds ratio was used by Chen and Thissen (1997).

$$
\operatorname{STDLN}\left(O R_{j j}\right)-\operatorname{RESID}=\frac{\ln \left[\frac{\left(n_{11}\right)\left(n_{00}\right)}{\left(n_{10}\right)\left(n_{01}\right)}\right]-\ln \left[\frac{E\left(n_{11}\right) E\left(n_{00}\right)}{E\left(n_{10}\right) E\left(n_{01}\right)}\right]}{\sqrt{\frac{1}{n_{11}}+\frac{1}{n_{10}}+\frac{1}{n_{01}}+\frac{1}{n_{00}}}}
$$

d. Mantel-Haenszel Statistic The Mantel-Haenszel statistic has been used as a measure effective for investigating DIF (Holland, 1985; Sinharay, 2006). The statistics has also used by Levy, Mislevy, \& Sinharay (2009) and by Levy (2011) to measure the bivariate response patterns of a pair of items:

$$
M H_{j j^{\prime}}=\frac{\sum_{r} n_{11 r} n_{00 r} / n_{r}}{\sum_{r} n_{10 r} n_{01 r} / n_{r}}
$$

where $n_{s s^{\prime}}$ denotes a count of examinees with response patterns that are conditional on rest score $r$, defined as the total test score excluding items $j$ and $j^{\prime}$. Levy, Mislevy, and Sinharay (2009) found that the Mental-Haenzel (MH) statistic is a sensitive measures capable of detecting in presence of local dependence due to multidimensionality.

e. $D_{j j^{\prime}}^{\chi}$ and $D_{j j^{\prime}}^{G}$ for Item Pairs The bivariate associations between item pairs have been measured by $X^{2}$ and $G^{2}$ types of statistics (e.g., Chen \& Thissen, 1997; Levy et al., 2009). The discrepancy measures are defined by 


$$
\begin{aligned}
& D_{j j^{\prime}}^{\chi}=\sum_{S=0}^{1} \sum_{S^{\prime}=0}^{1} \frac{\left(n_{S^{\prime}}-E\left(n_{s S^{\prime}}\right)\right)^{2}}{E\left(n_{s s^{\prime}}\right)} \text { and } \\
& D_{j j^{\prime}}^{G}=-2 \sum_{S=0}^{1} \sum_{S^{\prime}=0}^{1} n_{S S^{\prime}} \ln \frac{E\left(n_{s S^{\prime}}\right)}{n_{S S^{\prime}}}
\end{aligned}
$$

where $s$ is a binary response $s \in\{0,1\}$. Within Bayesian approach, the expected frequency of the item pairs, i.e., $E\left(n_{s s}\right)$ can be obtained by the posterior distribution.

f. Covariance and Correlation Levy (2011) demonstrated the effectiveness of several correlation measures in detecting item-pair associations, i.e., $C O V_{j j}, M B C_{j j}$, (Reckase, 1997). That is,

$$
\begin{gathered}
\operatorname{COV}_{j j^{\prime}}=\frac{\sum_{i=1}^{N}\left(Y_{i j}-\bar{Y}_{j}\right)\left(Y_{i j^{\prime}}-\bar{Y}_{j \prime}\right)}{N}=\frac{\left(n_{11}\right)\left(n_{00}\right)-\left(n_{10}\right)\left(n_{01}\right)}{N^{2}} \\
M B C_{j j^{\prime}}=\frac{\sum_{i=1}^{N}\left(Y_{i j}-E\left(Y_{i j}\right)\right)\left(Y_{i j^{\prime}}-E\left(Y_{i j}\right)\right)}{N}
\end{gathered}
$$

Levy (2011) also investigated the use of such discrepancy measures for item pairs and concluded that to the measures lead to greater posterior predictive $\mathrm{p}$-values for item pairs i.e. usefully reflect different dimensions. 


\section{Chapter 3}

\section{Methods}

In this chapter, we start by formulating the Bayesian approach to diagnostic models, paying particular attention to choosing prior distributions for the parameters. Markov chain Monte Carlo (MCMC) algorithms are used to approximate the posterior distribution of the model parameters. Sections $3.2-3.4$ provide the choice of priors for 2PL IRT, DINAs with various attribute models, and GDMs with constant/varying slopes. Section 3.5 shows how to estimate the model parameters by using the MCMC algorithm. Section 3.6 proposes three discrepancy measures of Bayesian model fit indices, which are then applied to the models. The research designs, including an empirical data example and two simulation studies, are provided in section 3.7. We have implemented the MCMC algorithms presented in this chapter by using OpenBUGS. Posterior predictive model checks were conducted in the R3.0.2 programming language. Appendix A and Appendix B contain the corresponding OpenBUGS and R codes.

\subsection{Bayesian Model Formulation}

Bayesian model formulations for estimating the model parameters include specifying a likelihood function for the set of item responses conditional upon the item and attribute parameters, and prior distributions for the parameters. To determine prior 
distributions, we assume that the item parameters are independent of the structural parameters of the attribute space, i.e., $p\left(\boldsymbol{v}_{0}, \boldsymbol{v}_{1}, \xi\right)=p\left(\boldsymbol{v}_{0}, \boldsymbol{v}_{1}\right) p(\boldsymbol{\xi})$ for 2PL IRT, $p(\boldsymbol{s}, \boldsymbol{g}, \boldsymbol{\alpha})=p(\boldsymbol{s}, \boldsymbol{g}) \boldsymbol{\pi}_{\boldsymbol{\alpha}}$ for the DINA models, and $p(\boldsymbol{\beta}, \boldsymbol{\gamma}, \boldsymbol{\alpha})=p(\boldsymbol{\beta}, \boldsymbol{\gamma}) \boldsymbol{\pi}_{\boldsymbol{\alpha}}$ for the GDMs.

In the following sections we present model formulations for the measurement and structural parts of the 2PL IRT, DINA models, and the GDMs.

\subsection{Bayesian Formulation for the 2PL IRT}

Let $Y_{j}$ denote a binary response for item $j$; i.e., $Y_{j} \in\{0,1\}$. In the two-parameter logistic IRT model (2PL IRT), the item response model is defined as

$$
\text { Logit }\left(Y_{j}=1 \mid v_{0 j}, v_{1 j}, \xi\right)=v_{0 j}\left(\xi-v_{1 j}\right) \text {. }
$$

The following prior distributions have been chosen for difficulty parameter $\left(v_{1 j}\right)$, discrimination parameter $\left(v_{0 j}\right)$ for item $j$, and ability parameter $(\xi)$ for each examinee in this study:

$$
\begin{gathered}
v_{1 j} \sim N(0,100), j=1, \ldots, J \\
v_{0 j} \sim N(0,100) I\left[v_{0 j} \in(0, \infty)\right], j=1, \ldots, J . \\
\xi \sim N(0,1),
\end{gathered}
$$

where $I\left[v_{0 j} \in(0, \infty)\right]$ indicates the discrimination parameter, $v_{0 j}$ is constrained to be positive values. The normal distributions with large variances lead to almost flat priors for item-level parameters. The prior for the ability parameter is fixed at $\mathrm{N}(0,1)$, out of an identifiability concern. 
Under the assumption of independence among the $K$ items, the prior distributions for $\boldsymbol{v}_{1}$ and $\boldsymbol{v}_{0}$ are as follows:

$$
p\left(\boldsymbol{v}_{1}\right)=\prod_{j=1}^{J} p\left(v_{1 j}\right) \text { and } p\left(\boldsymbol{v}_{0}\right)=\prod_{j=1}^{J} p\left(v_{0 j}\right)
$$

Therefore, the joint posterior distribution for the 2PL IRT model can be written as:

$$
p\left(\boldsymbol{v}_{1}, \boldsymbol{v}_{0}, \boldsymbol{\xi} \mid \boldsymbol{Y}\right) \propto L\left(\boldsymbol{Y}, \boldsymbol{v}_{1}, \boldsymbol{v}_{0}\right) p\left(\boldsymbol{v}_{1}\right) p\left(\boldsymbol{v}_{0}\right) p(\xi)
$$

\subsection{Bayesian Formulation for the DINAs}

\subsubsection{Item Parameters}

In the DINA model, we place prior distributions on the item parameters, i.e., the guessing $\left(g_{j}\right)$ and anti-slipping parameters $\left(a_{j}=1-s_{j}\right)$. Following Tseng (2010) we use the following priors for the item parameters:

$$
\begin{array}{r}
g_{j} \sim \operatorname{Beta}(1,2) \\
a_{j} \mid g_{j} \sim \operatorname{Uniform}\left(g_{j}, 1\right)
\end{array}
$$

We impose a constraint that $g_{j}$ is smaller than $a_{j}$. Note that the joint pdf for the two parameters can be expressed as follows:

$$
\begin{aligned}
p\left(a_{j}, g_{j}\right) & =p\left(a_{j} \mid g_{j}\right) p\left(g_{j}\right) \\
& =\frac{1}{1-g_{j}} \frac{\Gamma(3)}{\Gamma(1) \Gamma(2)}\left(1-g_{j}\right) \\
& =2, \text { where } 0 \leq g_{j} \leq a_{j} \leq 1 \\
& =0, \text { otherwise. }
\end{aligned}
$$


This leads to a flat prior on $\left(a_{j}, g_{j}\right)$ in their support. Under the assumption of independence among items, the prior distributions for $\boldsymbol{g}$ and $\boldsymbol{a}$ are

$$
p(\boldsymbol{g})=\prod_{j=1}^{J} p\left(g_{j}\right) \text { and } p(\boldsymbol{a} \mid \boldsymbol{g})=\prod_{j=1}^{J} p\left(a_{j} \mid g_{j}\right)
$$

Figure 3.1 Joint Probability Density Function of Anti-slip Parameter $\left(\boldsymbol{a}_{\boldsymbol{j}}\right)$ and Guessing Parameter $\left(\boldsymbol{g}_{j}\right)$

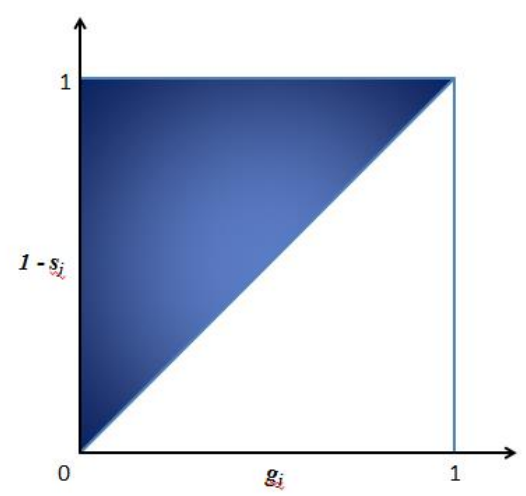

\subsubsection{Structural Parameters}

a. Independence Structure Let us suppose there are $K$ attributes; $\alpha_{k}$ indicates that an examinee has mastered or failed to master the corresponding attribute; i.e., $\alpha_{k} \in\{0,1\}$. Let $\pi_{\alpha_{k}}$ denote the probability that an examinee has mastered the attribute $k$ i.e. $\pi_{\alpha_{k}}=P\left(\alpha_{k}=1\right)$. Then the prior for each $\pi_{\alpha_{k}}$ is set to follow a uniform distribution:

$$
\pi_{\alpha_{k}} \sim \mathrm{U}(0,1)
$$


If we assume independence among $K$ attributes, then $\pi_{a}=\prod_{k=1}^{K} \pi_{\alpha_{k}}$. Therefore, the joint posterior distribution for the DINA model having the independence structure can be written as

$$
p(\boldsymbol{g}, \boldsymbol{a}, \boldsymbol{\alpha} \mid \boldsymbol{Y}) \propto L(\boldsymbol{Y}, \boldsymbol{g}, \boldsymbol{a}, \boldsymbol{\alpha}) p(\boldsymbol{a} \mid \boldsymbol{g}) p(\boldsymbol{g}) \boldsymbol{\pi}_{\boldsymbol{\alpha}} .
$$

b. Higher-order Structure Assuming that each component of the $\boldsymbol{a}$ (i.e. $\left.\alpha_{1}, \ldots, \alpha_{K}\right)$ can be associated with a general knowledge state (say $\theta$ ), the conditional probability of $\alpha_{k}$ given $\theta$ i.e., $\pi_{\alpha_{k} \mid \theta}$ can be modeled with an IRT by following

$$
\operatorname{Logit} \pi_{\alpha_{k} \mid \theta}=\lambda_{0 k}\left(\theta-\lambda_{1 k}\right)
$$

In this case, the following prior distributions for $\lambda_{0 k}$ and $\lambda_{1 k}$ for each attribute are

$$
\begin{gathered}
\lambda_{1 k} \sim N(0,1) \\
\lambda_{0 k} \sim N(0,1) I\left[\lambda_{0 k} \in(0, \infty)\right] \\
\theta \sim N(0,1),
\end{gathered}
$$

where $I\left[\lambda_{0 k} \in(0, \infty)\right]$ indicates the discrimination parameter, and $\lambda_{1 k}$ is constrained to be a positive value. Under conditional independence among $K$ attributes given $\theta$, the priors for the $\lambda_{1}, \lambda_{0}$ are

$$
p\left(\lambda_{1}\right)=\prod_{k=1}^{K} p\left(\lambda_{1 k}\right) \text { and } p\left(\lambda_{0}\right)=\prod_{k=1}^{K} p\left(\lambda_{0 k}\right) .
$$

Therefore, the joint posterior distribution for the model can be expressed as:

$$
\begin{gathered}
\quad p\left(\boldsymbol{g}, \boldsymbol{a}, \boldsymbol{\alpha}, \boldsymbol{\lambda}_{1}, \boldsymbol{\lambda}_{0}, \boldsymbol{\theta} \mid \boldsymbol{Y}\right) \\
\propto L(\boldsymbol{Y}, \boldsymbol{g}, \boldsymbol{a}, \boldsymbol{\alpha}) p(\boldsymbol{a} \mid \boldsymbol{g}) p(\boldsymbol{g}) \boldsymbol{\pi}_{\boldsymbol{\alpha}} p\left(\boldsymbol{\lambda}_{1}\right) p\left(\boldsymbol{\lambda}_{0}\right) p(\boldsymbol{\theta}) .
\end{gathered}
$$


c. Saturated Structure We place a prior distribution on the structural parameters of the saturated DINA model as follows. Let $\boldsymbol{\alpha}=\left(\alpha_{1}, \ldots, \alpha_{K}\right)^{T}$ be a vector of $K$ attributes, where each component of $\boldsymbol{\alpha}$ indicates that an examinee has mastered or failed to master the corresponding attribute. Because each attribute $\alpha_{k}$ can take only binary values, this leads to $2^{K}$ mutually exclusive patterns that can classify each examinee into one of the attribute patterns. Assuming that the $K$ attributes (i.e. $\alpha_{1}, \ldots, \alpha_{K}$ ) may be associated with other, we place priors on the attribute patterns; i.e., $\boldsymbol{\alpha}_{1}, \ldots, \boldsymbol{\alpha}_{L}$, where $L=2^{K}$ and $\sum_{l=1}^{L} \pi_{\boldsymbol{\alpha}_{l}}=1$. The categorical distribution is set to accommodate such conditions by following

$$
\boldsymbol{\alpha}_{l} \sim \text { Categorical }\left(\pi_{\boldsymbol{\alpha}_{1}}, \ldots, \pi_{\boldsymbol{\alpha}_{L}}\right),
$$

where $l=1, \ldots, L$. We recall that random variables $\boldsymbol{\alpha}_{1}, \ldots, \boldsymbol{\alpha}_{L}$ are mutually exclusive events with $P\left(\boldsymbol{\alpha}_{1}\right)=\pi_{\boldsymbol{\alpha}_{1}}, \ldots, P\left(\boldsymbol{\alpha}_{L}\right)=\pi_{\boldsymbol{\alpha}_{L}}$. Thus the probability mass function is given by

$$
\pi_{\boldsymbol{\alpha}_{1}, \ldots, \boldsymbol{\alpha}_{L}}=\pi_{\boldsymbol{\alpha}_{1}}^{I\left(\alpha=\boldsymbol{\alpha}_{1}\right)} \ldots \pi_{\boldsymbol{\alpha}_{L}}^{I\left(\alpha=\alpha_{L}\right)},
$$

where $I\left(\alpha=\boldsymbol{\alpha}_{l}\right)$ indicates 1 if $\alpha=\boldsymbol{\alpha}_{l}$ and 0, otherwise.

Given the categorical distribution for $\pi_{\boldsymbol{\alpha}_{l}}$, we are interested in the hyper-priors for $\pi_{\boldsymbol{\alpha}_{1}}, \ldots, \pi_{\boldsymbol{\alpha}_{L}}$, and thus need to choose a hyperprior distribution. The Dirichlet is chosen, for it is the conjugate distribution of the categorical distribution. Therefore,

$$
\begin{gathered}
\left(\pi_{\boldsymbol{\alpha}_{1}}, \ldots, \pi_{\boldsymbol{\alpha}_{L}}\right) \sim \operatorname{Dir}\left(m_{1}, \ldots, m_{L}\right) \\
m_{l}=0.5 .
\end{gathered}
$$


The choice of $m_{l}=0.5$ leads to the Jeffery's prior for $\pi_{\boldsymbol{\alpha}}$. Let us suppose that $\pi_{\boldsymbol{\alpha}_{l}}$ has a Dirichlet $(\boldsymbol{m})$ distribution with $2^{K}$ categories and a vector of parameters $m=$ $\left(m_{1}, m_{2}, \ldots, m_{L}\right)$. In that case then the density function is defined as:

$$
\operatorname{Dir}\left(\boldsymbol{\pi}_{\boldsymbol{\alpha}} \mid \boldsymbol{m}\right)=\frac{\Gamma\left(\sum_{l} m_{l}\right)}{\prod_{l=1}^{2 K} \Gamma\left(m_{k}\right)} \prod_{l=1}^{L} \pi_{\boldsymbol{\alpha}_{l}}^{m_{k}-1}
$$

where $m_{l}>0$ and $\sum_{l} \pi_{\boldsymbol{\alpha}_{l}}=1$. Therefore the joint posterior distribution for the DINA model with the saturated structure can be expressed

$$
p(\boldsymbol{g}, \boldsymbol{a}, \boldsymbol{\alpha} \mid \boldsymbol{Y}) \propto L(\boldsymbol{Y}, \boldsymbol{g}, \boldsymbol{a}, \boldsymbol{\alpha}) p(\boldsymbol{a} \mid \boldsymbol{g}) p(\boldsymbol{g}) \boldsymbol{\pi}_{\boldsymbol{\alpha}}
$$

\subsection{Bayesian Formulations for the GDMs}

\subsubsection{Item Parameters}

Let $Y_{j}$ denote a binary response for item $j$; i.e., $Y_{j} \in\{0,1\}$. If we assume a logit link function, the GDMs are defined as

$$
\operatorname{Logit} P\left(Y_{j}=1 \mid \beta_{j}, \gamma_{j}, \boldsymbol{\alpha}\right)=\beta_{j}+\gamma_{j}^{T} H\left(q_{j}, \boldsymbol{\alpha}\right)
$$

where $H\left(q_{j}, \boldsymbol{\alpha}\right)=\left(h_{1}\left(q_{j}, \boldsymbol{\alpha}\right), \ldots, h_{m}\left(q_{j}, \boldsymbol{\alpha}\right)\right)$ and where $h_{1}, \ldots h_{m}$ defines the interactions between $q$ and $\boldsymbol{\alpha}$. We use $H(, \boldsymbol{\alpha})=\left(q_{1 j} \alpha_{1}, \ldots, q_{K j} \alpha_{K}\right)$.

Under the varying slopes assumption, we use $H\left(q_{j}, \boldsymbol{\alpha}\right)=\left(q_{1 j} \alpha_{1}, \ldots, q_{K j} \alpha_{K}\right)$. That is,

$$
\text { Logit } P\left(Y_{j}=1 \mid \beta_{j}, \gamma_{j}, \boldsymbol{\alpha}\right)=\beta_{j}+\sum_{k=1}^{K} \gamma_{k j} q_{k j} \alpha_{k}
$$

Similarly, under the constant slopes assumption, we use $H\left(q_{j}, \boldsymbol{\alpha}\right)=\sum_{k=1}^{K} q_{k j} \alpha_{k}$. That is, 


$$
\text { Logit } P\left(Y_{j}=1 \mid \beta_{j}, \gamma_{j}, \boldsymbol{\alpha}\right)=\beta_{j}+\gamma_{j} \sum_{k=1}^{K} q_{k j} \alpha_{k}
$$

We choose almost flat priors for the item-level parameters. The priors for the intercept (i.e., $\left.\beta_{j}\right)$ are :

$$
\beta_{j} \sim N(0,100)
$$

Also, the priors for the slopes (i.e., $\gamma_{k j}$ or $\gamma_{j}$ ) are

$$
\begin{aligned}
& \gamma_{j k} \sim N(0,100) I(0, \infty), \text { for varying slopes } \\
& \gamma_{j} \sim N(0,100) I(0, \infty), \text { for constant slopes. }
\end{aligned}
$$

Under conditional independence among $J$ items, the priors for the $\lambda_{1}, \lambda_{0}$ are

$$
\begin{gathered}
\qquad p(\boldsymbol{\beta})=\prod_{j=1}^{J} p\left(\beta_{j}\right) \\
p(\boldsymbol{\gamma})=\prod_{j=1}^{J} p\left(\gamma_{j}\right) \text { for the constant slopes and } p(\boldsymbol{\gamma})=\prod_{k=1}^{K} \prod_{j=1}^{J} \gamma_{j k} \text { for the varying } \\
\text { slopes. }
\end{gathered}
$$

\subsubsection{Structural Parameters}

In this study, we consider only an unstructured attribute space for the GDMs. Therefore, we employ the same prior distributions used for the structural parameters of the DINA model having unstructured attribute space in section 3.3.2. This means that the joint posterior distribution for the GDMs with the saturated structure can be expressed as

$$
p(\boldsymbol{\beta}, \boldsymbol{\gamma}, \boldsymbol{\alpha} \mid \boldsymbol{Y}) \propto L(\boldsymbol{Y}, \boldsymbol{\beta}, \boldsymbol{\gamma}, \boldsymbol{\alpha}) p(\boldsymbol{\beta}) p(\boldsymbol{\gamma}) \boldsymbol{\pi}_{\boldsymbol{\alpha}} \operatorname{Dir}\left(\boldsymbol{\pi}_{\boldsymbol{\alpha}} \mid \boldsymbol{m}\right)
$$




\subsection{Estimation}

\subsubsection{MCMC algorithms using BUGS}

The Markov chain Monte Carlo (MCMC) is used to estimate the model parameters. The MCMC algorithm was implemented by using OpenBUGS 3.2.2 (Lunn, Spiegelhalter, Thomas, \& Best, 2009). The R package "R2OpenBUGS" (Sturtz, Ligges, \& Gelman, 2005) was used to run the program from within R 3.0.2 (R Core Team, 2013).

OpenBUGS uses Gibbs sampling algorithms to approximate the posterior distributions of the model parameters. Each parameter is updated based on its full conditional posterior distributions over a large number of iterations. When some of the full conditional distributions are difficult to obtain, however, the BUGS program incorporates additional techniques within the Gibbs steps. First the program makes extensive use of the "Metropolis-within-Gibbs" technique. When the full conditional distributions are not known forms, samples are taken from a proposal density, and then the ratio between the density with the candidate point and with the current point in the posterior distribution is evaluated (Chib \& Greenberg 1995). If the candidate value is accepted, it becomes the next value in the chain; otherwise, another candidate point is chosen.

\subsubsection{Implementation}

For each analysis, four chains were run; each ran for 25,000 iterations, and starting values were automatically generated by the OpenBUGS program. We used a thinning parameter of 10 and used the first half as burn-in period. The resulting 1,250 
iterations from each chain were pooled and randomly mixed, thus totaling 5,000 iterations after burn-in to be used as samples drawn from the posterior distribution of the parameters. The use of multiple chains and thinning serves to reduce the dependencies among the iterations and ensures adequate convergence to and mixing from the posterior distribution. Out of each sample from the posterior distribution of the parameters we generated a replicated dataset, giving us a total of 5,000 replicated datasets for each model.

\subsubsection{Model Diagnostic Criteria}

First, we ensure that the four Markov chains are mixed and reach convergence to the target distribution by creating trace and autocorrelation plots. In addition, Geweke (1992) and Gelman and Rubin (1995) tests are performed. The Geweke test (1992) compares whether the mean estimate of the MCMC outputs from the early part of the Markov chain is significantly different from that derived from the latter part of the chain for each parameter. The test is essentially a two-sample z-test; if the z-statistic is large enough to be rejected, this could be due to non-stationarity. The function "geweke.diag" under the R package entitled “coda” (Martyn, Nicky, Kate, and Karen, 2006) was used. The test was performed for each chain separately.

On the other hand, using the Gelman and Rubin (1995) test allows one to consider multiple chains at once, by comparing for each parameter the variation due to betweenchain and the variation due to within-chain. Let us denote within-chain variance by $W$ 
and between-chain variance by $B$ for each parameter of interest, $\psi$. Then the weighted average of the two variances is calculated as

$$
\widehat{\operatorname{Var}}(\psi)=\frac{T-1}{T} W+\frac{1}{T} B
$$

where $T$ is the number of iterations. As $T \rightarrow \infty$ the contribution of the $B$ drops radically

because of $\frac{1}{T}$, whereas the contribution of the $W$ becomes greater. Accordingly, Gelman and Rubin (1995) proposed the following statistic as a convergence diagnostic:

$$
\hat{R}=\sqrt{\frac{\widehat{\operatorname{Var}(\psi)}}{W}} .
$$

If the statistic $\hat{R}$ is close to 1 , this suggests that the multiple chains have reached a convergence. The function "gelman.diag" within the "coda" package (Martyn et al., 2006) was used.

\subsection{Model Fit Criteria}

\subsubsection{Generating Data from Posterior Predictive Distribution}

For each of the 5,000 samples drawn from the posterior distribution of the model parameters, we generated 5,000 replicated data sets for each model by using R 3.0.2. We began the data-generation steps by first sampling values for the attribute distributions rather than generating data using exactly the same estimates of the attribute pattern in the original data. We went through this step for the purpose of reducing dependence between the observed and the replicated data upon the same parameters. In other words, we started by simulating the parameters of the highest possible level of the attribute distribution, e.g., 
$\boldsymbol{\pi}_{\boldsymbol{\alpha}}=\left(\pi_{\boldsymbol{\alpha}_{1}}, \pi_{\boldsymbol{\alpha}_{2}}, \ldots, \pi_{\boldsymbol{\alpha}_{L}}\right)^{T}$ for unstructured DINA and GDMs; $\theta \sim N(0,1), \lambda_{0 k}$ and $\lambda_{1}$ for the higher-order DINA; $\pi_{\boldsymbol{\alpha}}=\left(\pi_{\boldsymbol{\alpha}_{1}}, \pi_{\boldsymbol{\alpha}_{2}}, \ldots, \pi_{\boldsymbol{\alpha}_{K}}\right)^{T}$ for the independence DINA; and $\theta \sim N(0,1)$ for the 2PL IRT. After the attribute parameters had been sampled, we simulated an attribute pattern for each examinee in the dataset, and we concluded by sampling the examinees' item responses.

\subsubsection{Proposed Discrepancy Measures}

In order to find unique aspects of the observed data and to ascertain if the current model can capture these features, we utilized the following discrepancy measures depending on the varying purposes of the tests.

a. Proportion Correct The first discrepancy measure proposed in this study is the distribution of proportion correct (e.g., Hambleton \& Han, 2004; Sinharay et al., 2006; Levy, 2006). Let $n$ denote the total number of examinees and $n_{j}$ denote the number of examinees answering exactly $j$ items correctly, where $j=0, \ldots, J$. Then, the proportion of examinees who obtained the observed sum-score $j$ is

$$
P C_{j}=\frac{n_{j}}{n}
$$

The proportion correct is the sufficient statistic for the ability parameter in the Rasch model (Masters and Right, 1984), meaning that the statistic provides the fullest information available from the data about the latent ability parameter. In other words, the proportion correct (or summed score) is one of the most basic characteristics of the data 
that more complicated latent variable models (e.g., 2PL IRT or CDMs) should be able to predict adequately.

b. Item-Pair Odds Ratio As mentioned in section 2.6.2, the item-pair odds ratio was used to detect violations of the local independence (LI) assumption in unidimensional item response theory (IRT) models (Chen and Thissen, 1997), and also found to be a useful discrepancy measure in detecting model-data misfit in the PPMC (Sinharay et al., 2006).

Let $n_{k k^{\prime}}$ denote the number of examinees scoring $k$ on item $j$ and $k^{\prime}$ on item $j^{\prime}$. Then the sample odds ratio is denoted by:

$$
O R_{j j^{\prime}}=\frac{\left(n_{11}\right)\left(n_{00}\right)}{\left(n_{10}\right)\left(n_{01}\right)}
$$

where $j \neq j^{\prime}$ and $k, k^{\prime} \in\{0,1\}$.

Generally speaking, latent variables models (e.g. factor analysis or item response theory) attempt to describe the correlated structures among observed response variables. Therefore, if such models fit the data properly, they should be able to reproduce the correlated structure among the item responses. One of the simplest ways to check for the presence of such a characteristic is to examine the bivariate pairwise associations between the item responses. Thus the purpose of using this measure in this study is to examine the extent to which 2PL IRT and various diagnostic models can reproduce particular structures among the observed item responses.

In CDMs, it is natural to assume that the predicted association among item responses is affected by (a) the Q-matrix, (b) how observed responses relate latent traits 
(i.e., measurement model), and (c) how attributes are structured by themselves (i.e., attribute model).

Figure 3.2 demonstrates a simplest example. Let us suppose there are four test items (i.e., $Y_{1}, \ldots Y_{4}$ ) measuring two attributes (i.e., $\alpha_{1}$ and $\alpha_{2}$ ). And suppose that the Qmatrix indicates that the first attribute, i.e., $\alpha_{1}$ is required in order to solve $Y_{1}, Y_{2}$, and $Y_{3}$ while the second attribute, i.e., $\alpha_{2}$ is required in order to solve $Y_{3}$ and $Y_{4}$. Let us also suppose that the two attributes (i.e., $\alpha_{1}$ and $\alpha_{2}$ ) are correlated. In this setup, if we assume the independence structure of the attributes i.e., that the two attributes are not correlated at all, then the model will fail to predict the association between $\left(Y_{1}, Y_{4}\right)$ and $\left(Y_{2}, Y_{4}\right)$.

c. Attribute-Pair Correlation In order to measure bivariate association between attribute pairs, we employed the "per attribute sum-scores" (Henson, Templin, and Douglas, 2007; Chiu, Douglas, and Li, 2009). Let us define a sum-score for an attribute as

$$
W_{i k}=\sum_{j=1}^{J} Y_{i j} q_{j k}
$$

for examinee $i$. Then $W_{i k}$ ranges from 0 to the total number of items that require attribute $k$, i.e. $W_{i k} \in\left[0, J_{k}\right]$, where $J_{k}$ denotes the total number of items for attribute $k$. The measure originally was used to cluster subjects into the proper underlying latent classes (Chiu, Douglas, and Li, 2009). In this study we calculated the Pearson product-moment correlation coefficients between the attribute sum-scores $W_{k}$ and $W_{k}$, to quantify the strength between pairs of attributes. 
Going back for a moment to the example seen in Figure 3.2, let us suppose that the Q-matrix indicates that the first attribute, i.e., $\alpha_{1}$, is required to solve $Y_{1}, Y_{2}$, and $Y_{3}$, while the second attribute, i.e., $\alpha_{2}$, is required for solving $Y_{3}$ and $Y_{4}$. If the two attributes (i.e., $\alpha_{1}$ and $\alpha_{2}$ ) are correlated, the per-skill sum scores $W_{1}$ and $W_{2}$, which reflect the observed summed scores for the two latent attributes, should be correlated as well. In this setup, if we use the attribute model having the constraint that the two attributes are not correlated (the independence attribute model), then the correlation between $W_{1}$ and $W_{2}$ will be much less.

Figure 3.2 Example to Demonstrate the Utility of the Item-Pair Odds Ratio and of the Attribute-Pair Correlation

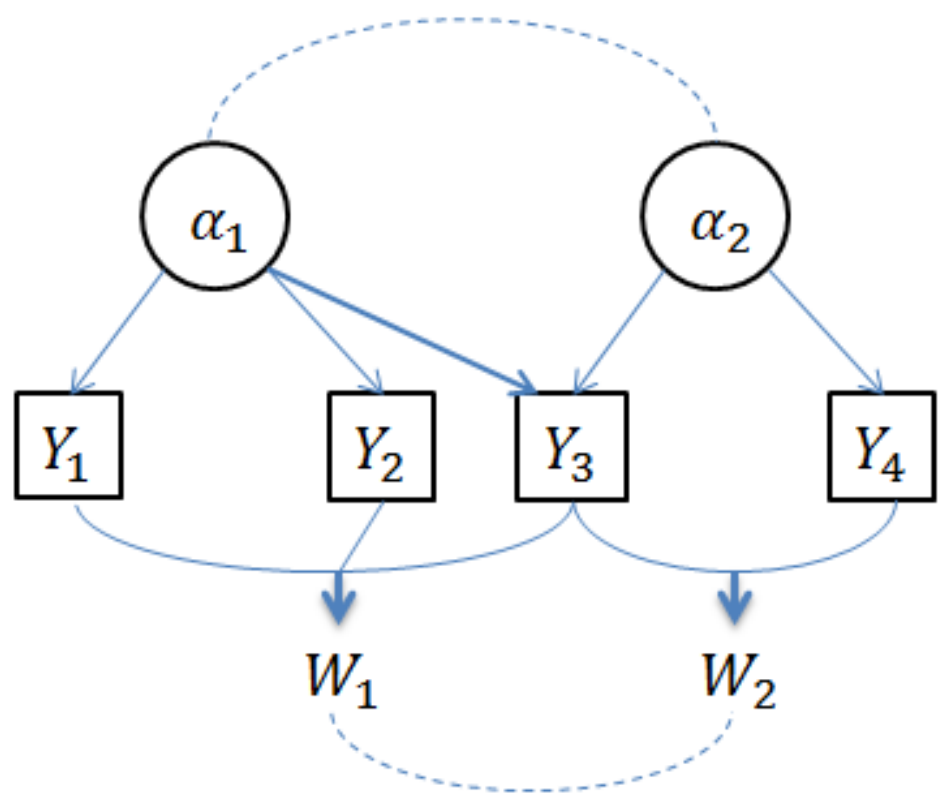




\subsection{Research Designs}

\subsubsection{Empirical Data Example}

To do the real data analysis, we used the fraction subtraction data (Tatsuoka, 1984), which is composed of responses to fraction subtraction test items responded to by middle school students. Each row of the data corresponds to the responses of a particular student $(1=$ correct response $/ 0=$ incorrect response). The item responses used for the fraction subtraction test originally appeared in Tatsuoka (1984); the dataset consisted of responses to 40 items made by 536 students. The data with the selected 20 test items measuring eight attributes were used by Tatsuoka (2002) and DeCarlo (2011). De la Torre (2009) selected 15 out of 20 items and recreated a Q-matrix having different combinations of five attributes in total. Figure 3.2 shows the distribution of total scores for the 15 items. The total score was the total number of items correctly answered by each individual.

As a preliminary analysis, we fitted the 2PL, DINAs, and GDMs to the fraction subtraction data for the purpose of comparing the models in terms of relative model fit indices. Results showed that 2PL IRT was preferred the most (BIC: 7099), followed by the DINA with unstructured model (BIC: 7295), the GDM with varying slopes (BIC: 7515), the DINA with higher-order model (BIC: 7740), the GDM with constant slopes (BIC: 7757), and the DINA with independence model (BIC: 7815). 
Table 3.1 Q-matrix Designed for Fraction Subtraction Data

\begin{tabular}{llccccc}
\hline Item No. & Item & $\alpha_{1}$ & $\alpha_{2}$ & $\alpha_{3}$ & $\alpha_{4}$ & $\alpha_{5}$ \\
\hline 1 & $3 / 4-3 / 8$ & 1 & 0 & 0 & 0 & 0 \\
2 & $31 / 2-23 / 2$ & 1 & 1 & 1 & 1 & 0 \\
3 & $6 / 7-4 / 7$ & 1 & 0 & 0 & 0 & 0 \\
4 & $3-21 / 5$ & 1 & 1 & 1 & 1 & 1 \\
5 & $37 / 8-2$ & 0 & 0 & 1 & 0 & 0 \\
6 & $44 / 12-27 / 12$ & 1 & 1 & 1 & 1 & 0 \\
7 & $41 / 3-24 / 3$ & 1 & 1 & 1 & 1 & 0 \\
8 & $11 / 8-1 / 8$ & 1 & 1 & 0 & 0 & 0 \\
9 & $34 / 5-32 / 5$ & 1 & 0 & 1 & 0 & 0 \\
10 & $2-1 / 3$ & 1 & 0 & 1 & 1 & 1 \\
11 & $45 / 7-14 / 7$ & 1 & 0 & 1 & 0 & 0 \\
12 & $73 / 5-4 / 5$ & 1 & 0 & 1 & 1 & 0 \\
13 & $41 / 10-28 / 10$ & 1 & 1 & 1 & 1 & 0 \\
14 & $4-14 / 3$ & 1 & 1 & 1 & 1 & 1 \\
15 & $41 / 3-15 / 3$ & 1 & 1 & 1 & 1 & 0 \\
\hline
\end{tabular}

Note. $\alpha_{1}=$ Subtract basic fractions; $\alpha_{2}=$ Reduce and simplify; $\alpha_{3}=$ Separate a whole number from fractions; $\alpha_{4}=$ Borrow from a whole number; $\alpha_{5}=$ Convert a whole number to fractions 
Figure 3.3 Distribution of Total Score (Fraction Subtraction Data)

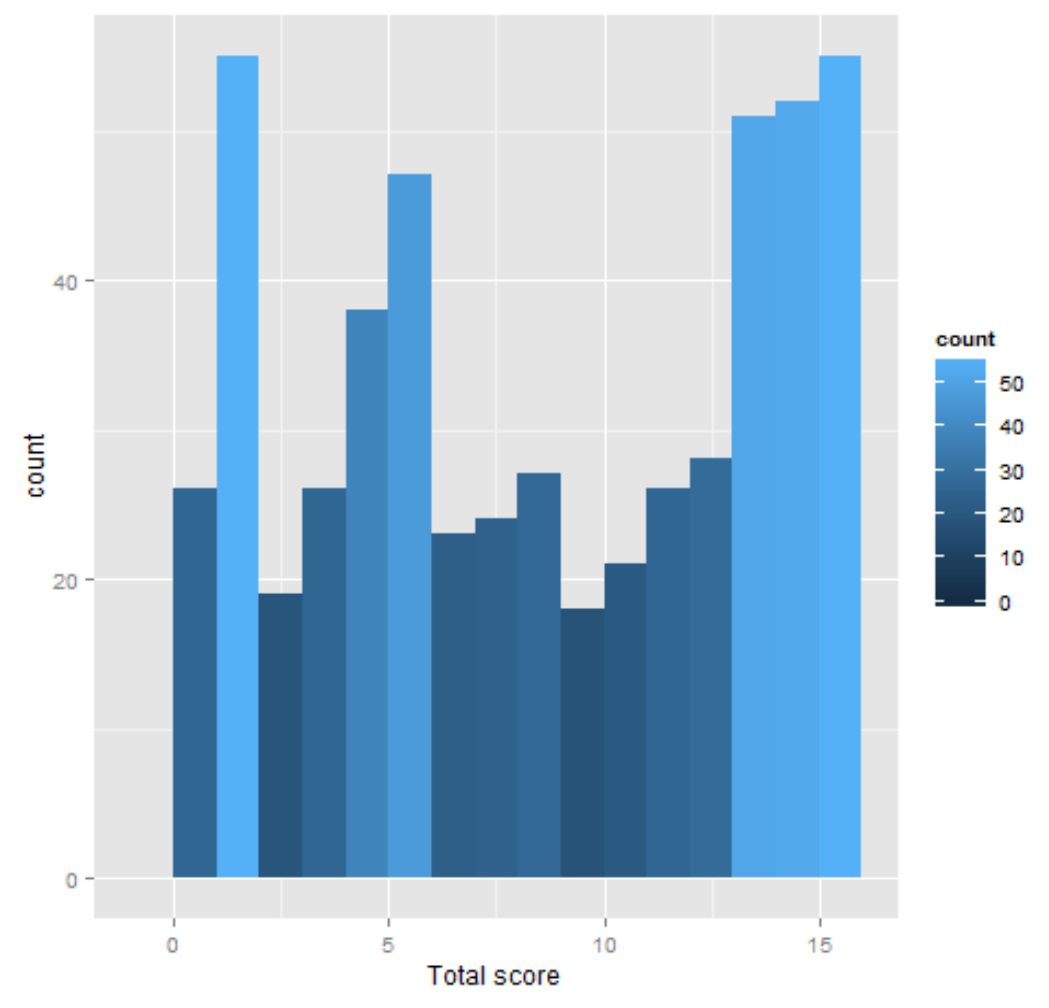




\subsubsection{Simulation Study I}

The main purpose of this study is to ascertain the extent to which unidimensional models (such as 2PL IRT) and multidimensional latent trait models (such as DINAs), based on a conjunctive rule space, can adequately predict a multidimensional compensatory structure of the data. Therefore, the GDM (with the assumption of varying slopes) is used as a data-generating model.

In order to simulate a realistic situation, we treat parameter estimates as a result of fitting the GDM to the same fraction subtraction data and treat the corresponding Qmatrix (Tatsuoka, 1985, de la Torre, 2009) as data-generating values. (Note that the data and the Q-matrix also were used in the empirical data example; see section 3.7.1). Specifically, we fit the GDM having varying slopes to the real data and generate a single replicated dataset by using the posterior distribution of the model parameter. The simulated dataset includes the binary responses of 536 examinees for 15 items. Next the 2PL IRT, DINAs with three attribute models (independence, higher-order, and unstructured), and the GDMs with constant and varying slopes are fitted to the dataset, after which the model-data misfit for each model is evaluated by using the proposed three discrepancy measures: total-score distribution, association between item pairs, and association between attribute pairs.

\subsubsection{Simulation Study II}

As will be discussed in section 4.1, we found based on the results of the empirical data example that the compensatory GDMs showed adequate model fit to the fraction 
subtraction data whereas the DINAs revealed a considerable lack of fit. Given that the fraction subtraction data has been a popular example dataset for demonstrating the various versions of the DINA model in many published articles, it is important to determine whether the types of misfit we discovered are in fact atypical for data actually generated from the DINA model. The simulation study we discuss below is meant to examine the expected behavior of the PPMC measures we have used here, when we know that the data actually have come from the DINA model.

Data-Generating Model In this study, data always have been generated from the DINA model having unstructured attribute space. Particularly we have been interested in seeing how each model performs when the strength between attribute pairs vary. In order to establish such conditions, we have considered the true attribute space in which attribute masteries are correlated weakly ( $\rho=0.2$ for each pair), moderately $(\rho=0.5$ for each pair), and strongly ( $\rho=0.8$ for each pair). Attribute mastery probabilities for the three attributes were set at 0.5. We use the R package "CDM" (Robitzsch, Kiefer, George, \& Uenlue, 2014) to do the data-generations.

The Q-matrix In order to mimic real data situations, we employed the Qmatrix designed for the Examination for the Certificate of Proficiency in English (ECPE; Buck \& Tatsuoka, 1998; Henson et al., 2007). The Q-matrix originally was constructed with three attributes (morphosyntactic, cohesive, and lexical attributes) to deal with 28 questions in total. In this study, the Q-matrix has been modified to create three sets of Qmatrices having different test lengths of 10, 20, and 30 (see Table 3.3). 


\section{True Item and Attribute Parameters Regardless of simulation}

conditions, the data-generating values for the guessing and the slip parameters have been fixed at 0.2 for all items in a test.

Sample Sizes and Test lengths Datasets are produced out of the datagenerating models, each of them containing the responses of 1,000 examinees to 10,20 , and 30 items.

Data-Analyzing Model Each dataset was analyzed in relation to a total of six models, i.e., the DINA models having the (a) independence model, (b) higher-order model, and (c) unstructured model; the two GDM models having the assumptions of (a) constant slopes and (b) varying slopes; and the 2PL IRT model. 
Table 3.2 Q-matrix Designed for Simulation Study II

\begin{tabular}{|c|c|c|c|c|c|c|}
\hline Item & $\alpha_{1}$ & $\alpha_{2}$ & $\alpha_{3}$ & 10 items & 20 items & 30 items \\
\hline 1 & 1 & 1 & 0 & $\mathrm{x}$ & $\mathrm{x}$ & $\mathrm{x}$ \\
\hline 2 & 0 & 1 & 0 & $\mathrm{x}$ & $\mathrm{x}$ & $\mathrm{x}$ \\
\hline 3 & 1 & 0 & 1 & $\mathrm{x}$ & $\mathrm{x}$ & $\mathrm{x}$ \\
\hline 4 & 0 & 0 & 1 & $\mathrm{x}$ & $\mathrm{x}$ & $\mathrm{x}$ \\
\hline 5 & 0 & 0 & 1 & $\mathrm{x}$ & $\mathrm{x}$ & $\mathrm{x}$ \\
\hline 6 & 0 & 0 & 1 & $\mathrm{x}$ & $\mathrm{x}$ & $\mathrm{x}$ \\
\hline 7 & 1 & 0 & 1 & $\mathrm{x}$ & $\mathrm{x}$ & $\mathrm{x}$ \\
\hline 8 & 0 & 1 & 0 & $\mathrm{x}$ & $\mathrm{x}$ & $\mathrm{x}$ \\
\hline 9 & 0 & 0 & 1 & $\mathrm{x}$ & $\mathrm{x}$ & $\mathrm{x}$ \\
\hline 10 & 1 & 0 & 0 & $\mathrm{x}$ & $\mathrm{x}$ & $\mathrm{x}$ \\
\hline 11 & 1 & 0 & 1 & . & $\mathrm{x}$ & $\mathrm{x}$ \\
\hline 12 & 1 & 0 & 1 & . & $\mathrm{x}$ & $\mathrm{x}$ \\
\hline 13 & 1 & 0 & 0 & . & $\mathrm{x}$ & $\mathrm{x}$ \\
\hline 14 & 1 & 0 & 0 & . & $\mathrm{x}$ & $\mathrm{x}$ \\
\hline 15 & 0 & 0 & 1 & . & $\mathrm{x}$ & $\mathrm{x}$ \\
\hline 16 & 1 & 0 & 1 & . & $\mathrm{x}$ & $\mathrm{x}$ \\
\hline 17 & 0 & 1 & 1 & . & $\mathrm{x}$ & $\mathrm{x}$ \\
\hline 18 & 0 & 0 & 1 & . & $\mathrm{x}$ & $\mathrm{x}$ \\
\hline 19 & 0 & 0 & 1 & . & $\mathrm{x}$ & $\mathrm{x}$ \\
\hline 20 & 1 & 0 & 1 & . & $\mathrm{x}$ & $\mathrm{x}$ \\
\hline 21 & 1 & 0 & 1 & . & . & $\mathrm{x}$ \\
\hline 22 & 0 & 0 & 1 & . & . & $\mathrm{x}$ \\
\hline 23 & 0 & 1 & 0 & . & . & $x$ \\
\hline 24 & 0 & 1 & 0 & . & . & $\mathrm{x}$ \\
\hline 25 & 1 & 0 & 0 & . & . & $\mathrm{x}$ \\
\hline 26 & 0 & 0 & 1 & . & . & $\mathrm{x}$ \\
\hline 27 & 1 & 0 & 0 & . & . & $\mathrm{x}$ \\
\hline 28 & 0 & 0 & 1 & . & . & $x$ \\
\hline 29 & 1 & 1 & 0 & . & . & $\mathrm{x}$ \\
\hline 30 & 0 & 1 & 0 & . & . & $\mathrm{x}$ \\
\hline
\end{tabular}


In this study we are interested in following hypotheses:

Hypothesis 1: The use of the Item-pair odds ratio will allow us to detect model failures to predict joint probabilities among item responses.

Hypothesis 1a. The 2PL IRT model will fail to predict the associations between the item pairs when the magnitudes of the attribute-pair relationships are weak.

The 2PL IRT model assumes independence among item responses conditional upon a unidimensional latent trait, whereas the data generated from the DINA model assume conditional independence among item responses upon multiple latent traits. Because weaker relationships between attribute pairs reflect more apparent multidimensionality, we expect that the 2PL IRT model will perform inadequately.

Hypothesis $1 b$. The DINA having the independence structure will fail to predict the associations between the item pairs if the magnitudes of the attribute-pair relationships are moderate or strong.

The independence structure of the attribute space assumes that attributes are independently distributed and do not affect each other. Because moderate or strong relationships between attribute pairs reflect that attributes are correlated, the independence model will fail to capture the true underlying structures.

Hypothesis 1c. The DINA with the higher-order structure will predict the associations between the item pairs adequately under most simulation conditions but will result in misfits if the magnitudes of the attribute-pair relationships are weak. 
The higher-order structure of the attribute space assumes that mastery of attributes is related to a unidimensional latent trait. Such a relationship would be valid when attributes are strongly correlated. If the attribute-pair relationships are weakly the assumption will not be supported and will reveal misfits.

Hypothesis 1d. The DINA with the saturated structure will predict the associations between the item pairs adequately under any simulation condition.

Because the data-generating model is the DINA model with the saturated structure, it is hypothesized that the model will predict the associations between item pairs adequately.

Hypothesis 1e. The GDM with the constant slope assumption will predict the associations between item pairs adequately under any simulation condition.

The measurement component of the GDM with the constant slope assumes that the observed item response is equally influenced by all the main effects of each required attribute, and thus the structure of the measurement model is less constrained than the measurement component of the DINA model. Also, since we use the saturated model for attribute space, there will be only a few misfits due to use of the attribute model.

Hypothesis 1f. The GDM with the varying slope assumption will predict all associations between item pairs.

The measurement component of the GDM with varying slopes assumes that the observed item response is affected by the main effects of the required attributes by varying amounts, in which case the structure of the measurement model will be less constrained than will the measurement component of the DINA model. Also, we use the saturated model for attribute space, so there will be no misfit. 
Hypothesis 2: Attribute-pair correlation coefficients will detect model failures and thereby predict the strength and direction of the associations between attribute pairs.

Hypothesis 2a. The 2PL IRT model will fail to predict the associations between attribute pairs adequately under most simulation conditions.

The 2PL IRT model assumes independence among item responses conditional on a unidimensional latent trait, whereas the data generated from the DINA model assume conditional independence among item responses on multiple latent traits. Because weaker relationships between attribute pairs reflect more apparent multidimensionality, it is hypothesized that the 2PL IRT model will fail to predict the attribute-pair correlation coefficients.

Hypothesis $2 \boldsymbol{b}$. The DINA having the independence structure will fail to predict the associations between attribute pairs adequately when those pairs are strongly or moderately associated.

The independence structure of the attribute space assumes that attributes are independently distributed and do not affect each other. Because moderate or strong relationships between attribute pairs assume the attributes are correlated, the assumption would fail to capture the true underlying structures.

Hypothesis 2c. The DINA having the higher-order structure will predict a few of the associations between attribute pairs adequately when those pairs are strongly or moderately correlated but will reveal misfits when they are weakly associated. 
The higher-order structure of the attribute space assumes that mastery of attributes is related to a unidimensional latent trait. Such a relationship will be valid when attributes are strongly correlated. If the attribute-pair relationships are weak, the structure will not be valid and will reveal misfits.

Hypothesis 2d. The DINA with the saturated structure will predict the associations between attribute pairs adequately under any simulation condition.

Because the data-generating model is the DINA model having a saturated structure, it is hypothesized that the model will reveal no misfits.

Hypothesis 2e. The GDM having the constant slope assumption will predict the associations between attribute pairs adequately under any simulation condition. .

The measurement component of the GDM having the constant slope assumes that the observed item response is equally influenced by the main effects of each required attribute, and hence the structure of the measurement model is less constrained than is the measurement component of the DINA model. Also, since we are using the saturated model for attribute space, there will be only few misfits.

Hypothesis $2 f$. The GDM having the varying slope assumption will predict the associations between attribute pairs adequately under all simulation conditions.

The measurement component of the GDM having the varying slope assumes that the observed item response is being affected by the main effects of the required attributes in varying amounts, and this suggests that the measurement component of the model is less constrained than the measurement component of the DINA model. Also, we use the saturated model for attribute space, so there will be no misfits. 


\section{Chapter 4}

\section{Results}

This chapter applies the PPMC method along with three proposed discrepancy measures to a real data example and two simulation studies. In section 4.1, we apply the methods to the fraction subtraction data (Tatsuoka, 1990). The data contain test responses for 15 items from a test given to 536 middle school students. A total of five attributes (de la Torre, 2009) was required to solve those 15 items. Section 4.2 presents the results of Simulation study I. In this study, its purpose is to examine model-data misfit when the data exhibit a compensatory structure. Also we used a compensatory GDM (with varying slope assumption) as our data-generating model. Also in the Simulation study I, we use a Q-matrix designed to account for the fraction subtraction data (see Table 3.1), which includes 15 item responses measuring 5 attributes.

In Simulation study II, we are interested in examining model-data misfit when data exhibit a conjunctive structure, hence our use of a DINA model as the datagenerating model. In this study we used the Q-matrix designed for the Examination for the Certificate of Proficiency in English (ECPE; Buck \& Tatsuoka, 1998; Henson et al., 2007) measuring three attributes in total. The Simulation study II seeks to assess the effect of test length $(10,20$, and 30 items) and the strength of attributes (weak, moderate, or strong) by using a 3-by-3 crossed design. 


\subsection{Result of Empirical Data Example}

\subsubsection{Model Convergence}

The 2PL IRT; the DINA models with (a) independence, (b) higher-order, and (c) unstructured models; and the GDM with (a) constant slopes and (b) varying slopes were all fitted to the fraction subtraction data. We first examined trace plots and autocorrelation plots to ensure that the Markov chain for each parameter of the six models has reached convergence; i.e., that the multiple Markov chains mixed properly and that the resulting samples can be treated as having been drawn from the stationary distribution. Detailed results of the Geweke (1992) and Gelman and Rubin (1992) tests are presented in Appendix C.

\subsubsection{Total Score Distribution}

Figure 4.2 compares the observed total score distribution with the corresponding posterior predictive distributions by fitting the six models to the fraction subtraction data. In the figure, the proportion of examinees for each observed total score are indicated by dots in black, and the posterior predictive means are presented as dots in grey with corresponding $90 \%$ posterior predictive intervals (dotted lines in grey). If the posterior predictive intervals do not contain the proportion of examinees associated with the observed total scores, this suggests that models are deficient when it comes to predicting the score distribution. The result suggests that overall, the six models perform similarly. It was found that choosing the unstructured or higher-order structure of the DINA model 
only moderately improved the accuracy in predicting the total-score distribution. It appears that the GDMs performed slightly better than did the DINAs in predicting the percentage of total examinees for each total score. 
Figure 4.1 Summary of the Posterior Predictive Distributions of the Observed Total Scores (Fraction Subtraction Data)
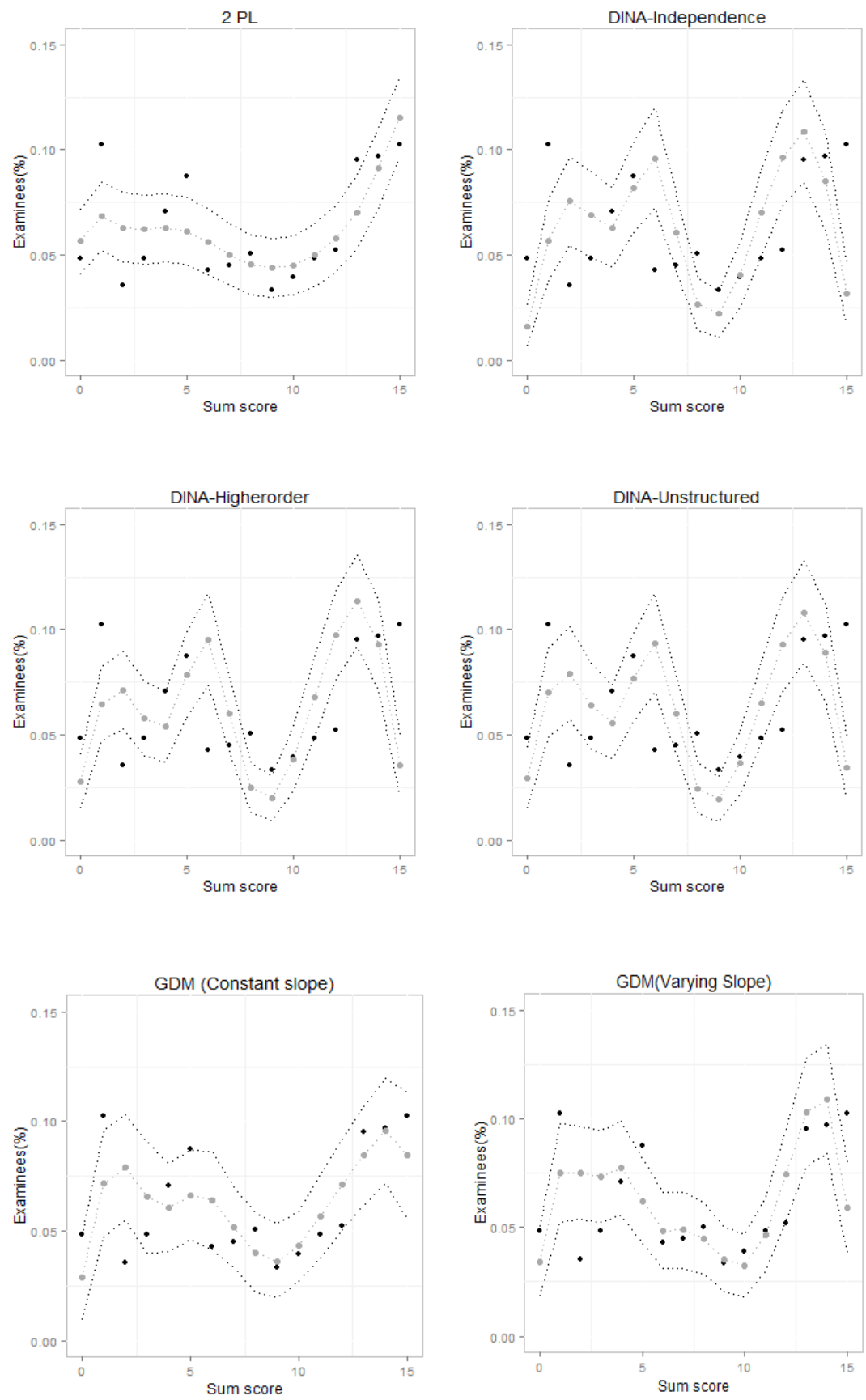

Note . Dots $($ black $)=$ Observed proportion of examinees; Dots $($ grey $)=$ Posterior mean for the proportion of examinees; Dotted line (grey) $=90 \%$ posterior predictive interval. 


\subsubsection{Association Between Item Pairs}

Figure 4.3 shows the posterior predictive p-values corresponding to the odds ratio for each item pair (with 15 items). The p-values are represented visually with heat maps, where color gradations represent the levels of the p-values. For maximal p-values between 1.00 and 0.95 the color is red, and for minimal p-values between 0.00 and 0.05 the color is blue. The p-values between 0.8 and 0.95 are colored in orange, those between 0.05 and 0.2 in light blue. Finally, the lightest color corresponds to moderate p-values, between 0.20 and 0.80 . If the posterior predictive p-value is extremely high, that means that the model tends to over-predict the item-pair association. Similarly if the p-value is extremely low, that means the model tends to under-predict the item-pair association. Note that the plot is symmetric with respect to the diagonal (top-left to right-bottom). Because we analyzed 15 items, there are a total of 105 pairs of associations to be considered.

2PL IRT As shown in Figure 4.3, the 2PL IRT model failed to predict item-pair odds ratios for 33 percent of the items (35 out of 105 pairs). Note especially that most of the misfit involved item 2 and item 3.

DINA models It appears that all three DINA models failed to predict many of the item-by-item associations. In particular, the DINA model with the independence structure failed to predict item-pair odds ratios too often when item 1 , item 5 , item 8 , and item 10 were involved. The four items led to extremely low $p$-values $(p \leq 0.05)$, meaning that the independence DINA model tended to under-predict the item-pair associations when involving the four items. The higher-order and unstructured DINA models showed 
better performances than did the independence DINA model but revealed a considerable amount of misfits in predicting the item-pair associations when item 1 or item 10 was involved. In sum, the DINA models resulted in extreme $p$-values $(p \leq 0.05)$ for item-pairs in nearly 50 percent of the time (52 out of 105 pairs) for the independence model, 38 percent of the time ( 40 out of 105 pairs) for the higher-order model, and 35 percent of the time (37 out of 105 pairs) for the unstructured model.

It is noticeable that the DINA models failed to predict most associations involving item 1 regardless of how the attribute space was modeled. Such a failure in the DINA models could occur because the conjunctive rule (i.e., examinees must possess all required attributes in order to answer the item correctly) that is inherent within the models is too restrictive. Item 1 however requires only attribute, i.e., $\alpha_{1}$ (=Subtract Basic Fractions), so it is less likely that the conjunctive rule will affect such a misfit. The failure in the DINA models also could result from the incorrectly specified Q-matrix for the item. As shown in the Q-matrix (Table 3.1), the item $1\left(\frac{3}{4}-\frac{3}{8}\right)$ requires only the attribute of subtracting fractions (i.e., $\alpha_{1}$ ). Apparently however "finding a common denominator" is another important attribute to solve this item; and yet the attribute is not one of the list of five attributes from the Q-matrix.

Chung (2014) proposed the use of a data-driven Q-matrix using the MCMC algorithm that is based on the DINA and the rRUM (reduced Reparametrized Unified Model; Hartz, 2002). The author found that the estimated Q-matrix based on the DINA suggested that the item $1\left(=\frac{3}{4}-\frac{3}{8}\right)$ only requires $\alpha_{2}$ (=Reduce and Simplify), which is 
different from what the original Q-matrix (Table 3.1) specified that the item requires only $\alpha_{1}$ (=Subtract Basic Fractions).

GDMs The GDM with the constant slopes resulted in 21 percent (22 out of 105 pairs) of the item pairs producing extreme $\mathrm{p}$-values $(\mathrm{p} \leq 0.05$ or $\mathrm{p} \geq 0.95)$. More specifically, nine of the extreme p-values were found to involve item 1 . And yet the GDM with varying slopes resulted in only 9 percent ( 9 out of 105 pairs) of the extreme pvalues. Allowing different slopes for each attribute significantly reduced the misfit involving item 1; there was only one misfit, i.e., item 1 by item 5 . In fact, the misfits involving item 5 were not reduced even after releasing the constraint on the slope parameters (i.e., using varying slopes) in the GDM. According to the expert Q-matrix (Table 3.1), the item requires only $\alpha_{5}$ (=Separate a Whole Number to a Fraction).

Two different studies have showed however that more attributes are needed to solve this item in the Fraction Subtraction data. DeCarlo (2012) assumed that there are uncertainties about $\alpha_{2}, \alpha_{4}, \alpha_{5}$ in order to solve item 5. Thus the author considered those elements of the Q-matrix to be random rather than fixed, and then estimated them by doing a Bayesian extension of the DINA model. The results suggest that $\alpha_{2}$ (=Reduce and Simplify) and $\alpha_{5}$ (=Convert a Whole Number to a Fraction) are necessary for the item, whereas $\alpha_{4}$ (=Borrow from a Whole Number) is not. Note that the author assumed that item 5 must need $\alpha_{1}$ (=Subtract Basic Fractions) and $\alpha_{3}$ (=Separate Whole Number from Fraction), and thus did not concern any uncertainty in regard to those two elements. Using a different technique within the Bayesian framework, Chung (2015) demonstrated an estimated Q-matrix suggesting the $\alpha_{3}$ (=Separate whole number from fraction) is not 
necessary, but $\alpha_{1}$ (=Subtract Basic Fractions) and $\alpha_{5}$ (=Convert Whole Number to Fraction) are sufficient to solve the item 5 correctly. 
Figure 4.2 Posterior Predictive P-values for Item-pair Odds Ratios (Fraction Subtraction Data)
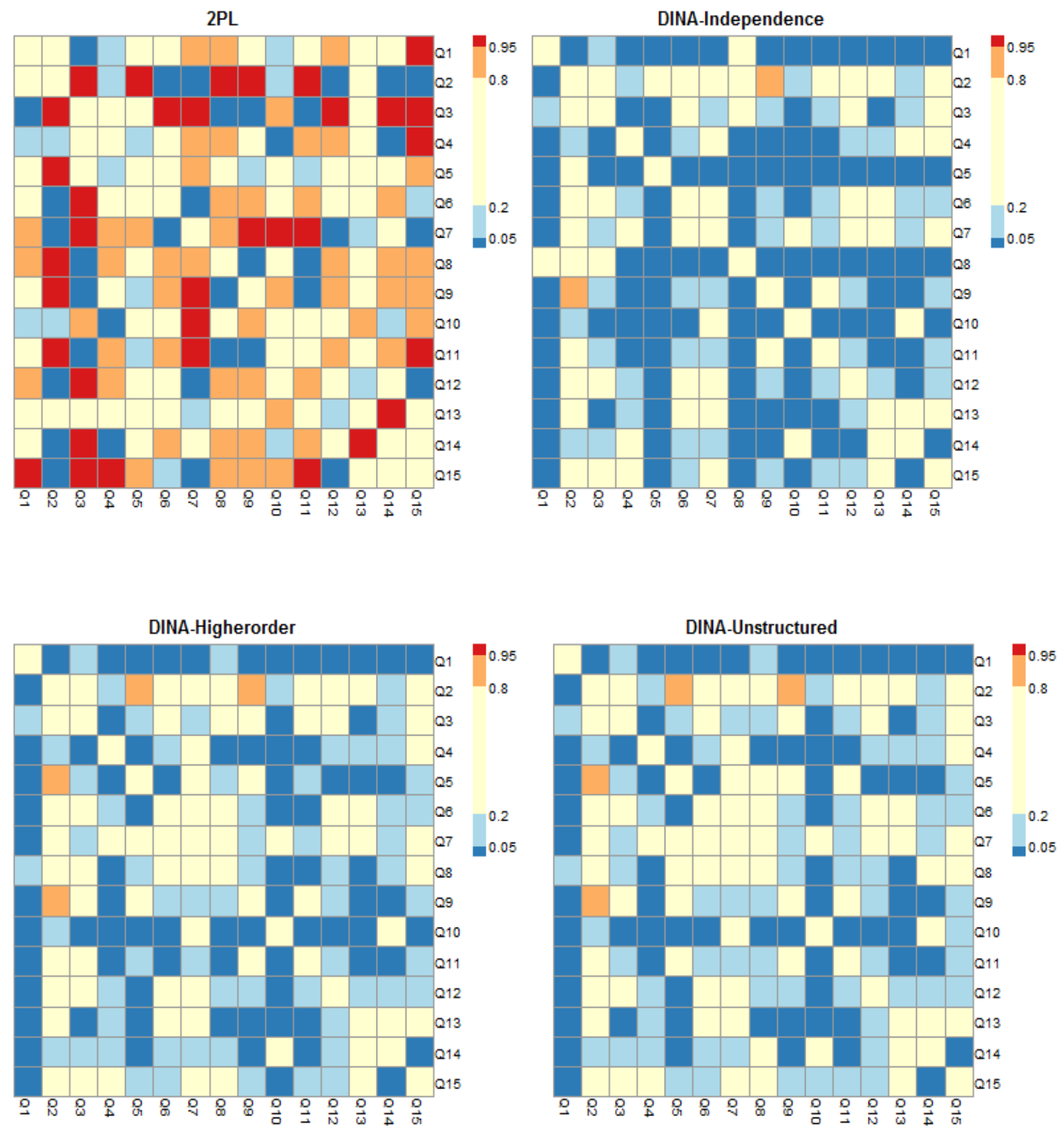
Figure 4.2 (Cont.) Posterior Predictive P-values for Item-pair Odds Ratios (Fraction Subtraction Data)
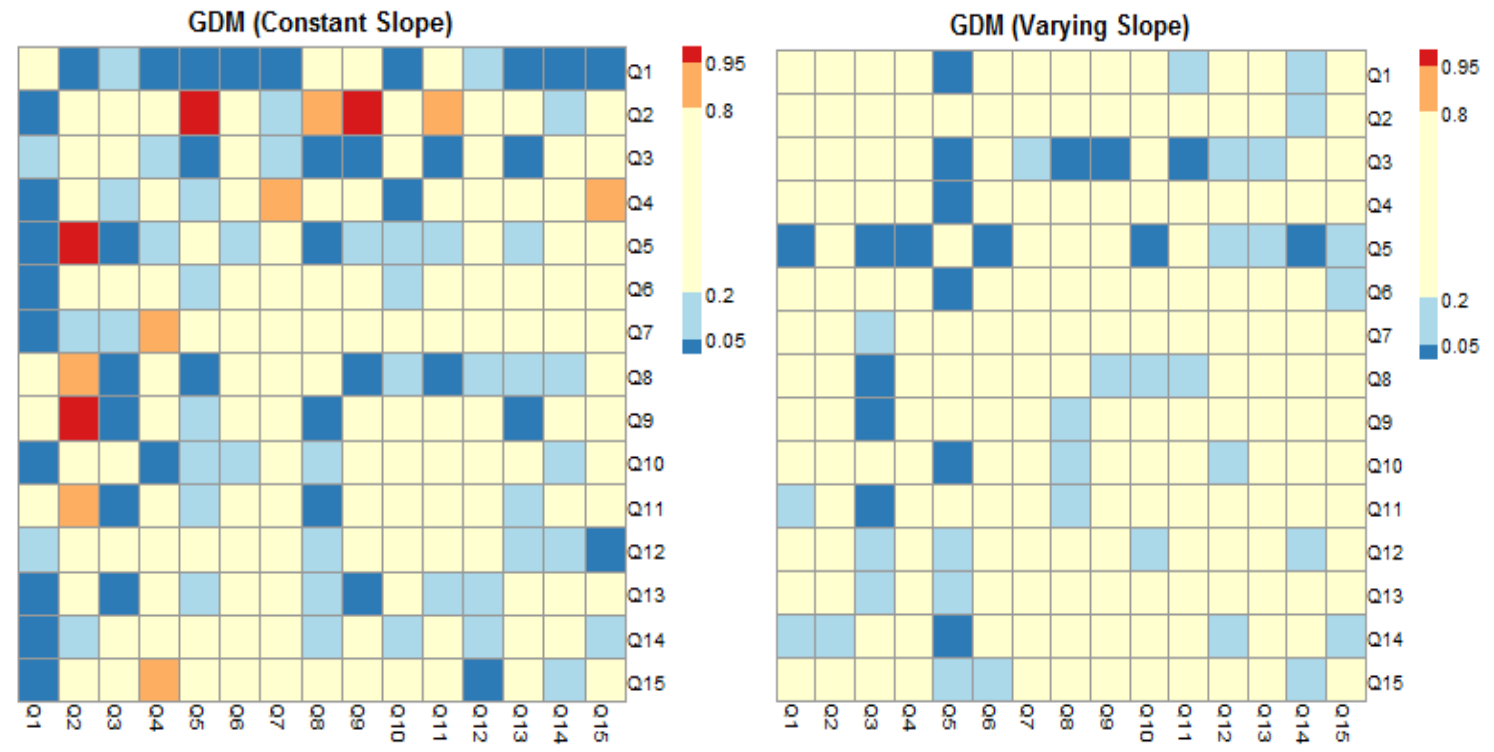


\subsubsection{Association Between Attribute Pairs}

Finally, attribute-pair correlations were evaluated by using the Pearson productmoment correlation coefficients between per-attribute sum score (Henson et al., 2007; Chiu et al., 2009). The posterior predictive p-values are shown in Table 4.1. The results suggest that the three DINA models with independence, higher-order, and unstructured models; all failed to predict associations between attribute masteries for nine to ten out of 10 pairs and the 2PL model failed for 7 out of the 10 pairs. As compared to the conjunctive models, the compensatory GDMs predicted the attribute-pair correlations more accurately. Use of the GDM with constant slopes led to four extreme p-values i.e. $\left(\alpha_{1}, \alpha_{2}\right),\left(\alpha_{1}, \alpha_{3}\right),\left(\alpha_{2}, \alpha_{3}\right)$, and $\left(\alpha_{2}, \alpha_{4}\right)$ out of 10 pairs. Finally, the GDM with the varying slopes for attribute led to only one extreme p-value, i.e., $\left(\alpha_{1}, \alpha_{4}\right)$.

Given these results, it appears that the compensatory GDMs we have examined provide the best fit to the fraction subtraction data. Given that the fraction subtraction data have served as a popular example dataset when it comes to demonstrating the various versions of the DINA model, it is important to determine if the types of misfit we have discovered are in fact atypical for data actually generated from the DINA model.

Simulation study II, which that we will be discussing in section 4.3 is meant to examine the expected behavior of the PPMC measures we have used here, when we know that the data actually have come from the DINA model. 
Table 4.1 Posterior Predictive P-values from the Pearson Product-Moment Correlation between Per Skill Sum Scores (Fraction Subtraction Data)

\begin{tabular}{|c|c|c|c|c|c|c|}
\hline \multirow[b]{2}{*}{ Pairs } & \multirow[b]{2}{*}{$2 \mathrm{PL}$} & \multicolumn{3}{|c|}{ DINA (Conjunctive) } & \multicolumn{2}{|c|}{$\underline{\text { GDM (Compensatory) }}$} \\
\hline & & Independence & Higherorder & Unstructured & $\begin{array}{c}\text { Constant } \\
\text { Slope }\end{array}$ & $\begin{array}{c}\text { Varying } \\
\text { Slope }\end{array}$ \\
\hline$\left(\alpha_{1}, \alpha_{2}\right)$ & 0.991 & $<.001$ & $<.001$ & $<.001$ & 0.020 & 0.495 \\
\hline$\left(\alpha_{1}, \alpha_{3}\right)$ & 0.849 & $<.001$ & $<.001$ & $<.001$ & $<.001$ & 0.149 \\
\hline$\left(\alpha_{1}, \alpha_{4}\right)$ & 1.000 & $<.001$ & $<.001$ & $<.001$ & 0.560 & 0.951 \\
\hline$\left(\alpha_{1}, \alpha_{5}\right)$ & 1.000 & $<.001$ & $<.001$ & 0.002 & 0.546 & 0.454 \\
\hline$\left(\alpha_{2}, \alpha_{3}\right)$ & 0.733 & 0.002 & 0.010 & 0.024 & 0.035 & 0.063 \\
\hline$\left(\alpha_{2}, \alpha_{4}\right)$ & 0.186 & $<.001$ & 0.004 & 0.002 & 0.031 & 0.181 \\
\hline$\left(\alpha_{2}, \alpha_{5}\right)$ & 1.000 & 0.016 & 0.030 & 0.035 & 0.492 & 0.308 \\
\hline$\left(\alpha_{3}, \alpha_{4}\right)$ & 1.000 & 0.041 & $<.001$ & 0.001 & 0.887 & 0.105 \\
\hline$\left(\alpha_{3}, \alpha_{5}\right)$ & 1.000 & 0.004 & 0.004 & 0.004 & 0.500 & 0.127 \\
\hline$\left(\alpha_{4}, \alpha_{5}\right)$ & 1.000 & 0.045 & 0.060 & 0.066 & 0.665 & 0.222 \\
\hline $\begin{array}{c}\text { \# Extreme } \\
\text { p-values }\end{array}$ & 7 & 10 & 9 & 9 & 4 & 1 \\
\hline
\end{tabular}




\subsection{Result of Simulation Study I}

\subsubsection{Total Score Distribution}

Figure 4.4 compares the observed total score distribution with the corresponding posterior predictive distributions by fitting the six models to a dataset generated from the GDM. If the posterior predictive intervals do not contain the proportion of examinees associated with the observed total scores, this suggests that the models are inadequate to predict the score distribution properly.

Overall, the results show that there was no lack of fit for either of the GDMs (varying and constant slopes) or the 2PL IRT model, when it comes to predicting the total score distribution. By contrast, the DINA models often failed to predict the percentages of examinees. In particular, the models tended to underestimate the number of students who obtain a perfect score $(=15)$; the predicted percentage for perfect score was $5 \%$ whereas the corresponding observed percentage for the score was $12 \%$. The models also failed to contain the observed percentage within the $90 \%$ posterior predictive intervals, and they underestimated the proportions for scores $0,1,8,9$, and 10. By contrast, the DINA model tended to over-predict the proportions for total scores 12 and 13. It was found that choosing the unstructured or higher-order structures of the DINA model only moderately improved its accuracy in predicting the total-score distribution. 
Figure 4.3 Summary of the Posterior Predictive Distributions of the Observed Total Scores (Simulation Study I)
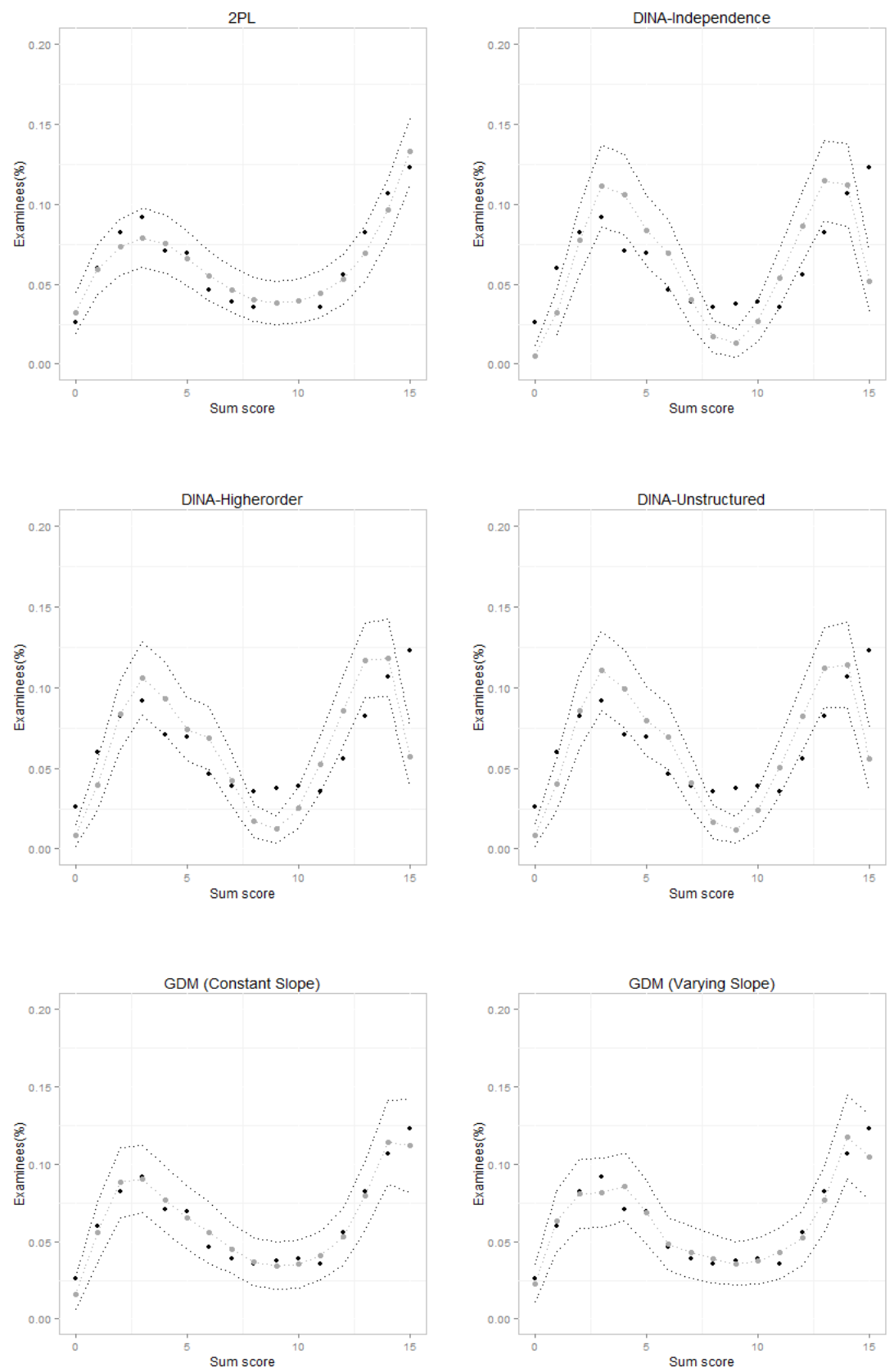

Note . Dots $($ black $)=$ Observed proportion of examinees; Dots $($ grey $)=$ Posterior mean for the proportion of examinees; Dotted line (grey) $=90 \%$ posterior predictive interval. 


\subsubsection{Association Between Item Pairs}

Figure 4.5 shows the posterior predictive p-values corresponding to the odds ratio for each item pair (with 15 items). The p-values are represented visually by heat maps, in which the color gradations represent the levels of the p-values. For maximal p-values between 1.00 and 0.95 the color is red, and for minimal p-values between 0.00 and 0.05 the color is blue. The p-values between 0.8 and 0.95 are colored in orange and those between 0.05 and 0.2 are colored in light blue. Finally, the lightest color corresponds to moderate p-values, between 0.20 and 0.80 . Note that the plot is symmetric with respect to the diagonal (top-left to right-bottom). Because we analyzed 15 questions, there are a total of 105 pairs of associations to be considered.

We can see from the figure that the $2 \mathrm{PL}$ model failed to find item-pair associations for 17 percent of the items (18 out of 105 pairs). In particular, item 2 caused a misfit that involved seven other items. It appears that all three DINA models also failed to predict many of the item-by-item associations. Specifically, the DINA model with an independence structure failed to predict 40 percent of the items (42 out of 105 pairs); 30 percent of the items ( 32 out of 105 pairs); 26 percent of the items ( 27 out of 105 pairs).

The GDM with the constant slopes resulted in 12 percent (13 out of 105 pairs) of the item pairs producing extreme $\mathrm{p}$-values ( $\mathrm{p} \leq 0.05$ or $\mathrm{p} \geq 0.95$ ), but the GDM with varying slopes did not reveal any of the extreme p-values. 
Figure 4.4 Posterior Predictive P-values for Item-pair Odds Ratios (Simulation Study I)
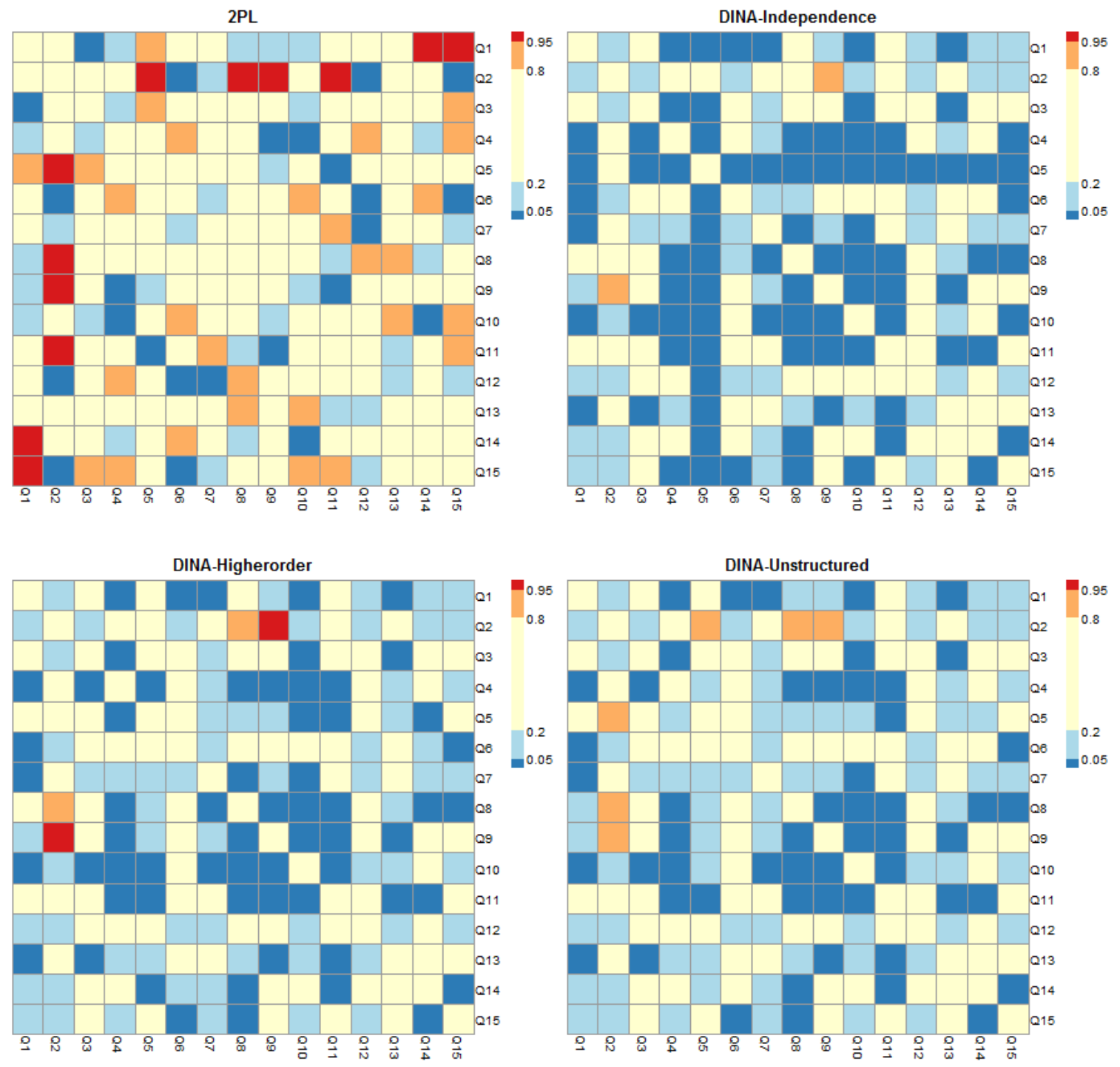
Figure 4.4 (Cont.) Posterior Predictive P-values for Item-pair Odds Ratios (Simulation Study I)
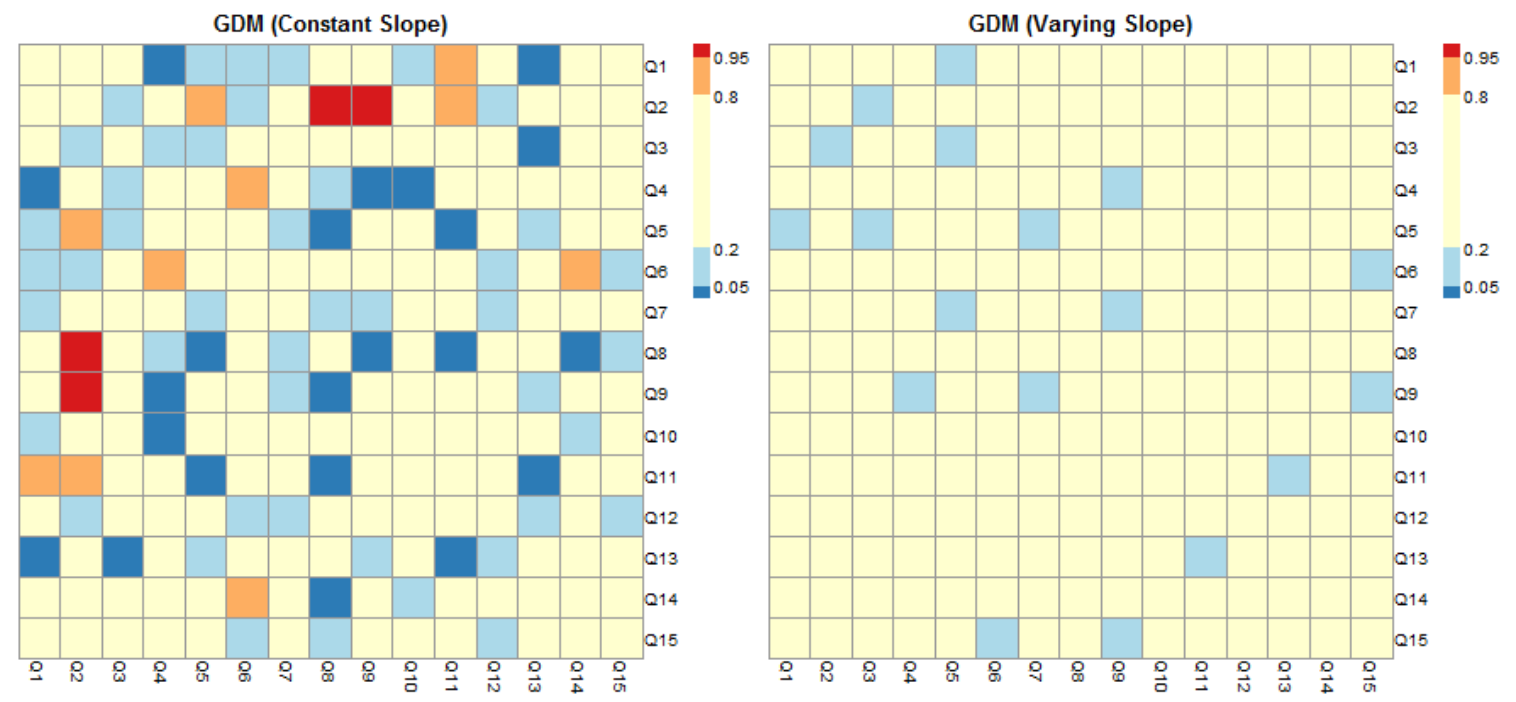


\subsubsection{Association Between Attribute Pairs}

Finally, attribute-pair correlations were evaluated by the Pearson product-moment correlation coefficients between 'per-skill sum score' (Henson et al., 2007; Chiu et al., 2009). The posterior predictive p-values are shown in Figure 4.6. Results suggest that the three DINA models with independence, higher-order, unstructured models failed to predict associations between attribute masteries for 6 to 7 out of the 10 pairs and the $2 \mathrm{PL}$ model for 8 out of the 10 pairs. Compared to the conjunctive models, compensatory GDMs predicted the attribute-pair correlations more accurately. The GDM with constant slopes led to three extreme p-values i.e. $\left(\alpha_{1}, \alpha_{2}\right),\left(\alpha_{2}, \alpha_{3}\right)$ and $\left(\alpha_{4}, \alpha_{5}\right)$, out of 10 pairs. Finally, the GDM with the varying slopes did not reveal any lack of fit. 
Figure 4.5 Posterior Predictive P-values from the Pearson Product-Moment Correlation between Per Skill Sum Scores (Simulation Study I)
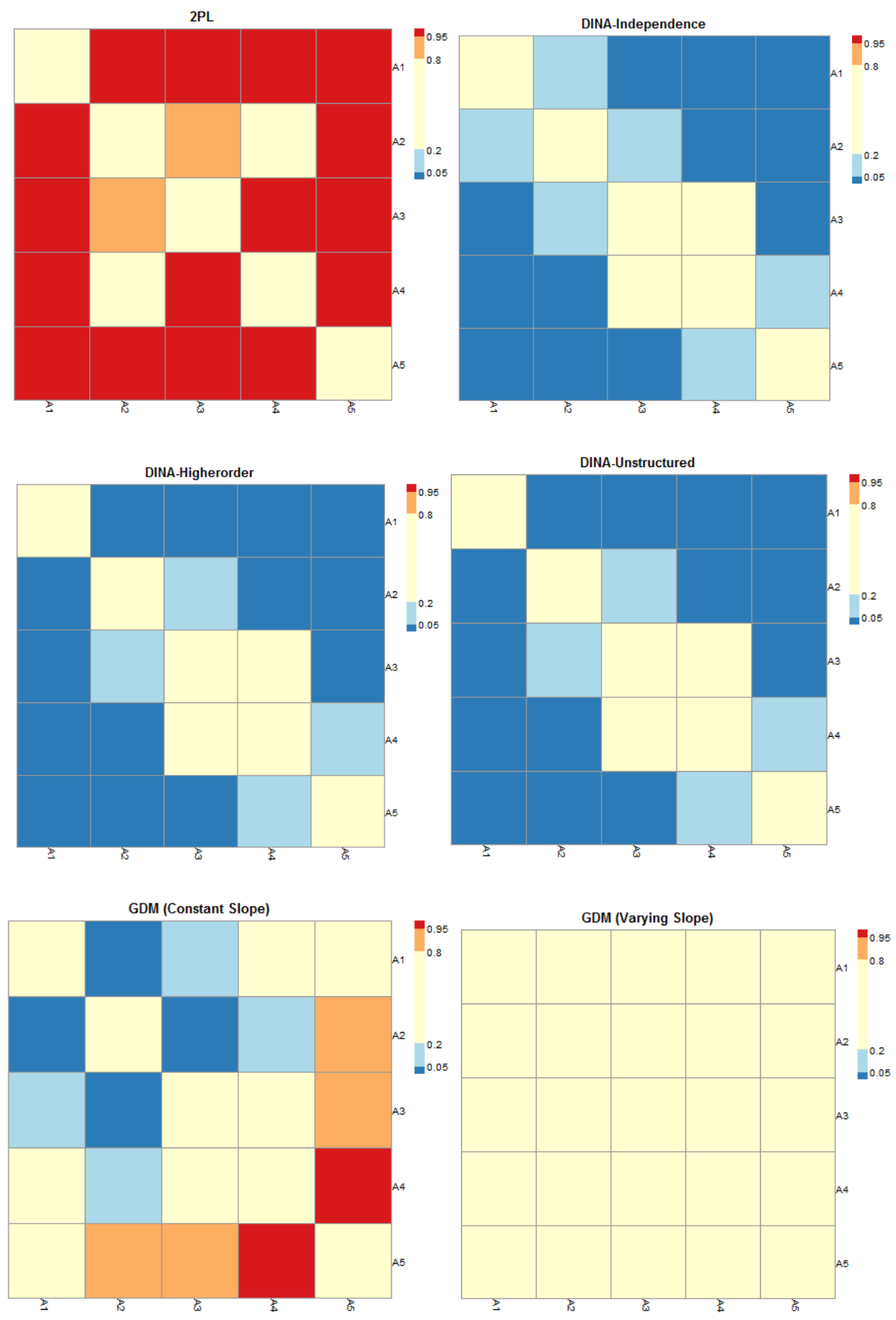


\subsection{Result of Simulation Results II}

\subsubsection{Association Between Item Pairs}

Posterior predictive p-values were calculated for the item-pair odds ratios using the 5,000 replicated datasets. Because test lengths varied (10,20, and 30 items), we considered p-values corresponding to $(10 \times 9) / 2=45,(20 \times 19) / 2=190$, and $(30 \times 29) / 2=$ 435 pairs of odds ratios, respectively. Table 4.2 shows the percentage of the p-values either greater than .95 or less than .05 . The numbers within parentheses refer to the pvalues either greater than .80 or less than .20 .

Hypothesis 1: The use of the Item-pair odds ratio will allow us to detect model failures to predict joint probabilities among item responses.

Hypothesis 1a. The 2PL IRT model will fail to predict the associations between the item pairs when the magnitudes of the attribute-pair relationships are weak.

The results suggested that the 2PL IRT model performs better in predicting the item-pair odds ratios as the strength between attribute masteries get stronger and as the test length decreases. When the strengths are weak to moderate, over 35 percent of p-values are more extreme than $(.05, .95)$ and 65 percent of them are more extreme than $(.20, .80)$. Therefore, the hypothesis is supported.

Hypothesis $1 \boldsymbol{b}$. The DINA having the independence structure will fail to predict the associations between the item pairs if the magnitudes of the attribute-pair relationships are moderate or strong.

The DINA with independent structure showed a great deal of model misfit when the strengths between the attributes were moderate to strong. Specifically, the model failed to 
predict more than 50 percent of the item-pair associations under such conditions. Therefore, the hypothesis is supported.

Hypothesis 1c. The DINA with the higher-order structure will predict the associations between the item pairs adequately under most simulation conditions but will result in misfits if the magnitudes of the attribute-pair relationships are weak.

The DINA with the higher-order structure revealed 1 to 9 percent of p-values more extreme than $(.05, .95)$ and 15 percent to 35 percent of $p$-values more extreme than $(.2, .8)$. The amount of lack of fit increased as the strength between attribute masteries became weaker. Therefore, the hypothesis is supported.

Hypothesis 1d. The DINA with the saturated structure will predict the associations between the item pairs adequately under any simulation condition.

The DINA with the saturated structure resulted in 1 to 7 percent of p-values more extreme than $(.05, .95)$ and 15 to 27 percent of p-values more extreme than $(.2, .8)$. The amount of lack of fit decreased as the strength between attribute masteries increased. Therefore, the hypothesis is partially supported.

Hypothesis 1e. The GDM with the constant slope assumption will predict the associations between item pairs adequately under any simulation condition.

The GDM with the constant slope assumption resulted in zero percent to 14 percent of pvalues more extreme than $(.05, .95)$ and 20 percent to 43 percent of p-values more extreme than $(.2, .8)$. The amount of lack of fit decreased as the strength between attribute masteries grew stronger. Therefore, the hypothesis is partially supported. 
Hypothesis $1 f$. The GDM with the varying slope assumption will predict all associations between item pairs.

The GDM with the constant slope assumption resulted in zero percent to 6 percent of pvalues more extreme than $(.05, .95)$ and 17 to 32 percent of p-values more extreme than $(.2, .8)$. It appears that the model fits from the unstructured DINA model and the GDM with varying slopes were the best; but the GDM with varying slopes tended to show mild over-fitting, when the associations between attribute pairs were strong and the test lengths are 20 or 30 . The hypothesis is partially supported. 
Table 4.2 Percentage of Extreme P-values for Item-Pair Odds Ratios (Simulation Study

II)

\begin{tabular}{ccccccccc}
\hline & & & \multicolumn{3}{c}{ DINA (Conjunctive) } & & \multicolumn{2}{c}{ GDM (Compensatory) } \\
\cline { 8 - 9 } \cline { 7 - 8 } Strength & \# Items & 2 PL & Independence & Higherorder & Unstructured & & $\begin{array}{c}\text { Constant } \\
\text { Slopes }\end{array}$ & $\begin{array}{c}\text { Varying } \\
\text { Slopes }\end{array}$ \\
\hline \multirow{3}{*}{ Weak } & 10 & $.38(.67)$ & $.20(.36)$ & $.09(.33)$ & $.07(.20)$ & & $.04(.24)$ & $.04(.17)$ \\
& 20 & $.46(.72)$ & $.13(.35)$ & $.07(.32)$ & $.04(.26)$ & & $.09(.29)$ & $.01(.21)$ \\
& 30 & $.44(.69)$ & $.14(.39)$ & $.04(.32)$ & $.03(.24)$ & & $.06(.35)$ & $.06(.32)$ \\
\hline \multirow{5}{*}{ Moderate } & 10 & $.36(.69)$ & $.53(.67)$ & $.04(.29)$ & $.07(.24)$ & & $.02(.24)$ & $.02(.24)$ \\
& 20 & $.36(.59)$ & $.38(.62)$ & $.01(.16)$ & $.02(.15)$ & & $.14(.43)$ & $.03(.21)$ \\
& 30 & $.41(.66)$ & $.45(.63)$ & $.04(.23)$ & $.03(.20)$ & & $.03(.28)$ & $.03(.29)$ \\
\hline \multirow{3}{*}{ Strong } & 10 & $.18(.47)$ & $.64(.73)$ & $.02(.24)$ & $.02(.27)$ & & $.00(.20)$ & $.00(.24)$ \\
& 20 & $.18(.39)$ & $.54(.73)$ & $.01(.15)$ & $.01(.17)$ & & $.02(.24)$ & $.04(.23)$ \\
& 30 & $.23(.54)$ & $.62(.76)$ & $.01(.23)$ & $.01(.23)$ & & $.02(.30)$ & $.05(.31)$ \\
\hline
\end{tabular}

Note. Number indicates the percentage of posterior predictive p-values greater than .95 or less than .05 . Numbers within parentheses indicates the p-values greater than .80 or less than .20. 


\subsubsection{Association Between Attribute Pairs}

In order to examine the model-data misfit of the diagnostic models in predicting bivariate relationships between pairs of attribute masteries, $\mathrm{p}$-values were calculated for the attribute-pair correlation coefficients using the posterior predictive distribution based on the 5,000 replicated datasets (Table 4.3).

Hypothesis 2: Attribute-pair correlation coefficients will detect model failures and thereby predict the strength and direction of the associations between attribute pairs.

Hypothesis 2a. The 2PL IRT model will fail to predict the associations between attribute pairs adequately under most simulation conditions.

As expected, the 2PL model failed to predict almost all attribute-pair associations; almost all of the p-values were found to be greater than .95 (nearly 1), which implies that it consistently over-predicted the associations between pairs of attribute masteries. Therefore, the hypothesis is supported.

Hypothesis $2 \boldsymbol{b}$. The DINA having the independence structure will fail to predict the associations between attribute pairs adequately when those pairs are strongly or moderately associated.

The DINA model with independence structure consistently under-predicted the associations. When the relations between attribute masteries were moderate to strong, the p-values were found to be nearly zero. The model also failed to predict them when the true attributes were weakly related. Therefore, the hypothesis is partially supported. 
Hypothesis 2c. The DINA having the higher-order structure will predict a few of the associations between attribute pairs adequately when those pairs are strongly or moderately correlated but will reveal misfits when they are weakly associated.

The higher-order model performed sufficiently well to predict all possible associations, and there were no extreme p-values; the p-values ranged from .08 to .81 . Therefore, the hypothesis has not been supported.

Hypothesis 2d. The DINA with the saturated structure will predict the associations between attribute pairs adequately under any simulation condition.

The p-values from the unstructured DINA model range from .32 to .61 across all simulation conditions. Therefore, the hypothesis is supported.

Hypothesis 2e. The GDM having the constant slope assumption will predict the associations between attribute pairs adequately under any simulation condition. The GDM with constant slopes revealed a few misfits in the relations between attribute 1 and attribute 3. The model tended to under-predict the relations when the underlying condition was weak or moderate and test lengths were 20 or 30, but it over-predicted the relations when the underlying condition was strong. Beyond those conditions, the pvalues range from .31 to .61 . Therefore, the hypothesis is partially supported.

Hypothesis 2f. The GDM having the varying slope assumption will predict the associations between attribute pairs adequately under all simulation conditions.

The GDM with varying slopes under-predicted the relation between attribute 1 and attribute 3 when the true attribute-pairs were weakly correlated and the test length was 20 but over-predicted those associations when the true attribute-pairs were strongly 
correlated and the test length was 20 . Under the remaining conditions, the p-values range from .21 to .79 . Therefore, the hypothesis is partially supported. 
Table 4.3 Posterior Predictive P-values from the Pearson Product-Moment Correlation between Per-Skill Sum Scores (Simulation Study II)

\begin{tabular}{|c|c|c|c|c|c|c|c|c|}
\hline \multirow[b]{2}{*}{ Strength } & \multirow[b]{2}{*}{ \# Items } & \multirow[b]{2}{*}{ Pair } & \multirow[b]{2}{*}{$2 \mathrm{PL}$} & \multicolumn{3}{|c|}{ DINA (Conjunctive) } & \multicolumn{2}{|c|}{ GDM (Compensatory) } \\
\hline & & & & Independence & Higherorder & Unstructured & $\begin{array}{c}\text { Constant } \\
\text { Slopes } \\
\end{array}$ & $\begin{array}{c}\text { Varying } \\
\text { Slopes } \\
\end{array}$ \\
\hline \multirow{9}{*}{ Weak } & \multirow{3}{*}{10} & $\left(\alpha_{1}, \alpha_{2}\right)$ & 0.22 & 0.08 & 0.73 & 0.46 & 0.33 & 0.32 \\
\hline & & $\left(\alpha_{1}, \alpha_{3}\right)$ & 1.00 & 0.00 & 0.11 & 0.40 & 0.38 & 0.44 \\
\hline & & $\left(\alpha_{2}, \alpha_{3}\right)$ & 0.98 & 0.03 & 0.78 & 0.43 & 0.33 & 0.35 \\
\hline & \multirow{3}{*}{20} & $\left(\alpha_{1}, \alpha_{2}\right)$ & 0.99 & 0.06 & 0.70 & 0.51 & 0.33 & 0.22 \\
\hline & & $\left(\alpha_{1}, \alpha_{3}\right)$ & 1.00 & 0.00 & 0.10 & 0.25 & 0.13 & 0.25 \\
\hline & & $\left(\alpha_{2}, \alpha_{3}\right)$ & 1.00 & 0.10 & 0.76 & 0.50 & 0.46 & 0.23 \\
\hline & \multirow{3}{*}{30} & $\left(\alpha_{1}, \alpha_{2}\right)$ & 1.00 & 0.02 & 0.74 & 0.47 & 0.27 & 0.26 \\
\hline & & $\left(\alpha_{1}, \alpha_{3}\right)$ & 1.00 & 0.03 & 0.08 & 0.39 & 0.03 & 0.03 \\
\hline & & $\left(\alpha_{2}, \alpha_{3}\right)$ & 1.00 & 0.00 & 0.86 & 0.50 & 0.34 & 0.33 \\
\hline \multirow{9}{*}{ Moderate } & \multirow{3}{*}{10} & $\left(\alpha_{1}, \alpha_{2}\right)$ & 0.83 & 0.00 & 0.59 & 0.55 & 0.39 & 0.30 \\
\hline & & $\left(\alpha_{1}, \alpha_{3}\right)$ & 1.00 & 0.00 & 0.21 & 0.36 & 0.52 & 0.75 \\
\hline & & $\left(\alpha_{2}, \alpha_{3}\right)$ & 1.00 & 0.00 & 0.42 & 0.45 & 0.39 & 0.45 \\
\hline & \multirow{3}{*}{20} & $\left(\alpha_{1}, \alpha_{2}\right)$ & 1.00 & 0.00 & 0.54 & 0.35 & 0.25 & 0.21 \\
\hline & & $\left(\alpha_{1}, \alpha_{3}\right)$ & 1.00 & 0.00 & 0.12 & 0.45 & 0.01 & 0.75 \\
\hline & & $\left(\alpha_{2}, \alpha_{3}\right)$ & 1.00 & 0.00 & 0.73 & 0.49 & 0.28 & 0.31 \\
\hline & \multirow{3}{*}{30} & $\left(\alpha_{1}, \alpha_{2}\right)$ & 1.00 & 0.00 & 0.54 & 0.35 & 0.32 & 0.33 \\
\hline & & $\left(\alpha_{1}, \alpha_{3}\right)$ & 1.00 & 0.00 & 0.12 & 0.45 & 0.18 & 0.19 \\
\hline & & $\left(\alpha_{2}, \alpha_{3}\right)$ & 1.00 & 0.00 & 0.73 & 0.49 & 0.36 & 0.41 \\
\hline \multirow{9}{*}{ Strong } & \multirow{3}{*}{10} & $\left(\alpha_{1}, \alpha_{2}\right)$ & 0.92 & 0.00 & 0.28 & 0.48 & 0.45 & 0.42 \\
\hline & & $\left(\alpha_{1}, \alpha_{3}\right)$ & 1.00 & 0.00 & 0.36 & 0.45 & 0.58 & 0.61 \\
\hline & & $\left(\alpha_{2}, \alpha_{3}\right)$ & 0.99 & 0.00 & 0.12 & 0.41 & 0.36 & 0.47 \\
\hline & \multirow{3}{*}{20} & $\left(\alpha_{1}, \alpha_{2}\right)$ & 1.00 & 0.00 & 0.42 & 0.57 & 0.72 & 0.50 \\
\hline & & $\left(\alpha_{1}, \alpha_{3}\right)$ & 1.00 & 0.00 & 0.40 & 0.45 & 0.92 & 0.99 \\
\hline & & $\left(\alpha_{2}, \alpha_{3}\right)$ & 1.00 & 0.00 & 0.39 & 0.61 & 0.55 & 0.65 \\
\hline & \multirow{3}{*}{30} & $\left(\alpha_{1}, \alpha_{2}\right)$ & 1.00 & 0.00 & 0.26 & 0.32 & 0.42 & 0.35 \\
\hline & & $\left(\alpha_{1}, \alpha_{3}\right)$ & 1.00 & 0.00 & 0.31 & 0.43 & 0.61 & 1.00 \\
\hline & & $\left(\alpha_{2}, \alpha_{3}\right)$ & 1.00 & 0.00 & 0.44 & 0.55 & 0.47 & 0.79 \\
\hline
\end{tabular}


Figure 4.6 Summary of Extreme P-values from Item-Pair Odds Ratio and Pearson Product-Moment Correlations between Per-Skill Sum Scores (Simulation study II)
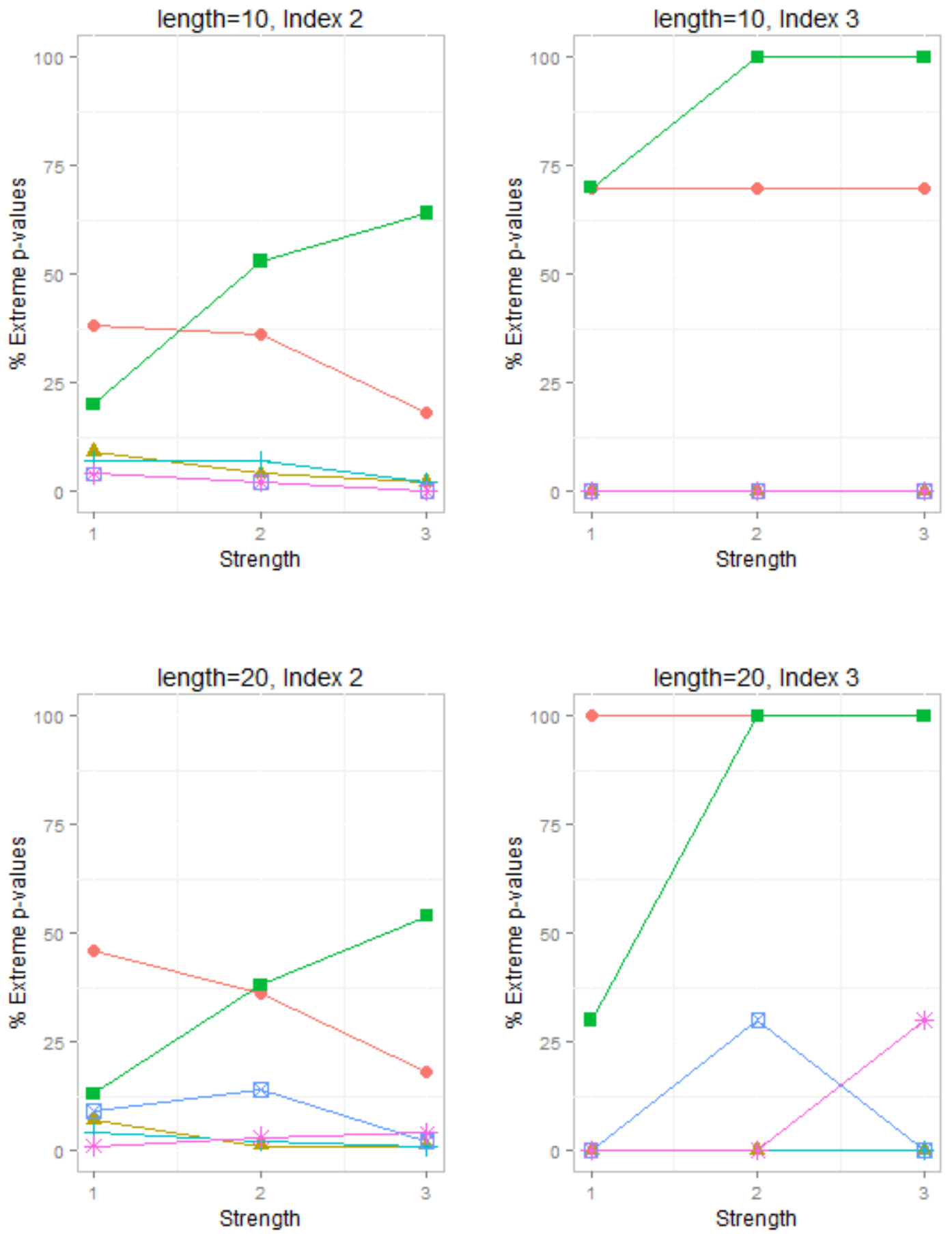
Figure 4.6 (Cont.) Summary of Extreme P-values from Item-Pair Odds Ratio and Pearson Product-Moment Correlations between Per-Skill Sum Scores (Simulation study II)
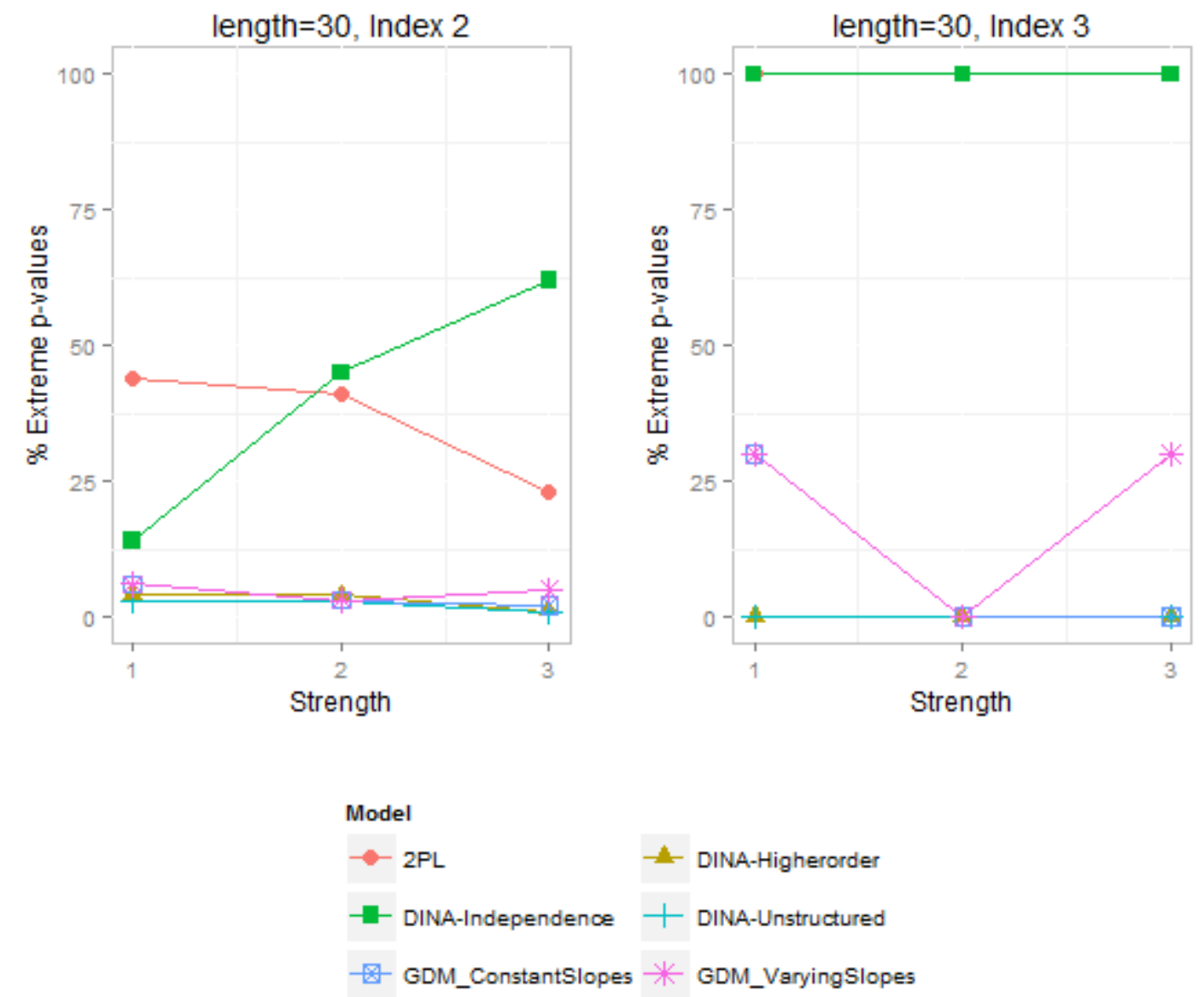

Note. $\mathrm{y}$-axis indicates percentages of extreme $\mathrm{p}$-value (posterior predictive $\mathrm{p}$-values that are greater than .95 or less than .05). Index 2: Item-pair odds ratio; Index 3: Pearson correlation coefficient between per-attribute sum scores. 


\section{Chapter 5}

\section{Discussion}

\subsection{Implications}

Even since the introduction of CDMs back in the 1990s, these models have received increasing attention from educational researchers. Despite their burgeoning popularity, however, there are still not enough studies that seek to evaluate model-data misfit. PPMC has been considered an attractive method of detecting various aspects of model-data misfit within the psychometric models (e.g., Sinharay et al., 2006; Levy, 2006, 2011; Sinharay, 2005), but the technique has not been fully applied to a wide variety of diagnostic models. It is precisely this gap in the research that the current study has sought to fill. We have explored the extent to which each model is capable of predicting some systematic aspects of the data structure. We have considered diagnostic models by viewing them through the perspectives of both conjunctive and compensatory rules. Also, we have examined the impact of inadequately defined attribute structures on the DINA model.

Three discrepancy measures were used: total-score distributions, odds ratios between item pairs, and correlations between attribute pairs. The observed total-score distribution was first examined within the real data example; this was a simple, graphical way of determining whether shape of response function can be replicated by applying the 
DINA models to the real data example. The odds ratio as an item-fit measure has been widely deemed as a powerful discrepancy measure to detect associations among item pairs (e.g., IRT, MIRT, and BN). Therefore, the measure was used as we continued the real data analysis and the two simulation studies in the current study. In the real data example, the posterior predictive p-values associated with this measure were efficiently presented by using a visualization technique. Finally, we introduced correlation coefficients between per attribute sum-scores (Henson et al., 2007; Chiu et al., 2009) as a way of checking the associations between attribute pairs. This allowed us to detect correlations among the attributes and thereby see if the model being studied was capable of reproducing the directions and strengths of the attribute pairs.

The data analysis example with the fraction subtraction data revealed that the DINA model showed a considerable lack of fit in this case. More particularly, assuming independence among attributes was found to be an overly restrictive approach to modeling the attribute space. In diagnostic tests such as fraction subtraction data, it often is the case that there are certain types of correlations or dependences among the attributes; e.g., attribute A cannot be mastered until attribute B has been mastered (Corter, 1995). Therefore, it is natural that the independent structure among attributes fails to predict the underlying structure of the diagnostic assessment data often. Quite simply, the models failed to predict true associations between item pairs and attribute pairs.

It also was noticeable that the 2PL IRT performed better than the DINA models did whereas the compensatory GDMs with constant slopes and varying slopes showed only a minimal amount of misfit. Several reasons are possible explanations for why the 
conjunctive models (i.e. DINAs) do not fit the data properly, the mostly likely of them being that use of the conjunctive rule constitutes an overly restrictive approach when only one Q-matrix is being considered (Tatsuoka, 1990). In cognitive assessments, it is likely that examinees instead choose one of the various possible strategies to solve problems in order to maximize the accuracy of problem solving and minimize the cognitive load (Rho, 2010).

The simulation studies suggested that the independent structure of the DINA model has serious misfits when the correlation among attributes is moderate to strong. The higher-order model worked almost as well as the unstructured model, perhaps because we considered only three attributes under the simulation conditions. Ideally, the goal is to find a structural model that is parsimonious yet adequately describes the true attribute space. The two GDMs performed well under most conditions but revealed overfittings under a few particular conditions.

After one has discovered particular model misfits, one must take all possible actions to compensate for them. At the very least it has become clear that the approach, modifying structural aspects of the DINA model, is not the only way to address model misfit. Sinharay (2007) suggests that if a particular model is going to be chosen as the final model for a given dataset, then particular items that produce serious misfits with the model should be removed and the model should be fitted with the remaining items, thereby ensuring an adequate model fit using PPMC. Depending upon which discrepancy measure is being used and how sensitive it is, occasions will arise when at least some defects are found in the finalized model. 
Thus it is important to emphasize that practitioners should be aware of the defects and the possible consequences when they are choosing models and make inferences (Gelman et al., 1996).

\subsection{Limitations and Future Research}

The current study has some limitations that will need to be addressed in the future. First, the implications to be drawn from the two simulation studies are valid only for particular data structures. In other words, Simulation studies I and II focused only on conditions in which datasets are simulated from the unstructured attribute spaces of the GDMs and the DINA models. Future researches may wish to examine other datagenerating models considering different measurement models (e.g., GDM with constant slope) and structural models (e.g., higher-order structure or independent structure).

Second, Simulation study II found only that the pairwise attribute masteries within the attribute space have constant and positive correlations; i.e., that is, each of the pairwise correlation coefficients is $0.2 \mathrm{~s}$ for weak association, $0.5 \mathrm{~s}$ for moderate association, and $0.8 \mathrm{~s}$ for strong association. In real data the correlations between different attribute pairs could be different and even negative for some of the pairs. The present study's setup, the study easily demonstrated situations in which the independent structure of the DINA model causes a lack of fits. Such conditions were not however exhaustive enough to allow us to determine precisely when the higher-order structure of the DINA model is going to fail. Thus in future research, it will be necessary to investigate the performance of the higher-order model in the presence of more complex dependence 
structure. Examples include conditions whereby (a) the bonds between the attribute masteries vary by attribute pairs; (b) some of the attribute masteries are negatively correlated; and (c) test items measure more high-dimensional structures. Similarly, in our study all of the generating values for the guessing and slip parameters were fixed at 0.2 for all of the items, and therefore the impact of different item-level parameters on the model fit indices has not been discussed (For example high guessing parameters, which would make the effects of attribute mastery less significant.)

Also, because we have been interested in potential lack of fit due to the dichotomized scale of the attributes, we divided the individual cognitive-attributes into two categories, mastery and non-mastery; that has perhaps been too restrictive and certainly has led to loss of information. Thus there is scope for future researches where we consider polytomous attribute space is explored more fully, particularly by using the GDMs that take into account both non-binary attribute space and the Q-matrix.

For future research, PPMC methods need to be developed that are capable of detecting other aspects of lack-of-fit. The current study has demonstrated the significant degree to which the measurement and structural components of the models produce particular aspects of the model-data misfit. Although we have not determined the degree to which the performance of the diagnostic models depends on the degree of uncertainty of the expert-designed Q-matrix, discrepancy measure could be developed that would address the lack of accuracy in determining misspecifications when using the expertdesigned Q-matrix. Also, it is desired to employ some types of model fit indices that can penalize the models. Finally, new methods of quantifying the magnitudes of 
discrepancies, other than calculating the posterior predictive p-value, could be arrived at through future research. Indeed, Wu, Yuen, and Leung (2014) already have proposed a relative entropy posterior predictive model checking method (RE-PPMC). This utilizes information drawn from the whole distribution to measure the difference between the realized and the predictive distribution using relative entropy. 


\section{Bibliography}

Akaike, H. (1974) 'A new look at the statistical model identification'. IEEE Transactions on Automatic Control. Vol. 19, No. 6, pp 716-723.

Alagoz-Ekici, C. (2012) 'Posterior predictive model checking for the Diagnostic Input Noisy and Gate Model', University of Georgia (Ph.D. dissertation).

Baker, F.B. (1992) Item response theory, New York, NY, Marcel Dekker

Birnbaum, A. (1968). Some latent trait models and their use in inferring an examinee's ability. In F. M. Lord \& M. R. Novick (Eds.), Statistical theories of mental test scores (pp. 397—472). Reading, MA: Addison-Wesley

Buck, G. and Tatsuoka, K.K. (1998) 'Application of the rule-space procedure to language testing: examining attributes of a free response listening test', Language Testing, Vol. 15, No. 2, pp.119-157.

Chen, W-H. and Thissen, D. (1997) 'Local dependence indexes for item pairs using item response theory', Journal of Educational and Behavioral Statistics, Vol. 22, No. 3, pp.265-289.

Chib, S. and Greenberg, E. (1995) 'Understanding the Metropolis-Hastings algorithm', The American Statistician, Vol. 49, No. 4, pp.327-335.

Chiu, C., Douglas, J. and Li, X. (2009) 'Cluster analysis for cognitive diagnosis: theory and applications', Psychometrika, Vol. 74, No. 4, pp.633-655.

Chung, M. (2014) 'Estimating the Q-matrix for Cognitive Diagnosis Models in a Bayesian Framework', Columbia University (Ph.D dissertation). 
Corter, J. E. (1995). Using clustering methods to explore the structure of diagnostic tests. In P. Nichols, S. Chipman \& R. Brennan (Eds.), Cognitively Diagnostic Assessment. Hillsdale NJ: Lawrence Erlbaum Associates, 305-326.

Dayton, C.M. \& Macready, G.B. (1988) 'Concomitant-variable latent class models', Journal of the American Statistical Association, Vol. 83, No.401, pp173-178. de la Torre, J. (2009) 'DINA model and parameter estimation: a didactic', Journal of Educational and Behavioral Statistics, Vol. 34, No. 1, pp.115-130.

de la Torre, J. and Douglas, J. (2004) 'Higher-order latent trait models for cognitive diagnosis', Psychometrika, Vol. 69, No. 3, pp.333-353.

Decarlo, L.T. (2011) 'On the analysis of fraction subtraction data: the DINA model, classification, latent class sizes, and the Q-matrix', Applied Psychological Measurement, Vol. 35, No. 1, pp8-26.

DiBello, L., Roussos, L. and Stout, W. (2007) 'Review of cognitively diagnostic assessment and a summary of psychometric models', in C.R Rao and S. Sinharay (Eds.): Handbook of Statistics, Vol. 26, pp.979-1030, Elsevier, Amsterdam.

Gelman A., Carlin, J.B., Stern, H.S. and Dunson, D.B. (2013) Bayesian Data Analysis, 3rd ed., Chapman \& Hall, New York.

Gelman, A., Meng, X.L. and Stern, H.S. (1996) 'Posterior predictive assessment of model fitness via realized discrepancies (with discussion)', Statistica Sinica, Vol. 6, pp.733-807.

Gelman, A. and Rubin, D.B. (1992) 'Inference from iterative simulation using multiple sequences', Statistical Science, Vol. 7, No. 4, pp457-511. 
Geman, S. and Geman, D. (1984) 'Stochastic relaxation, gibbs distributions, and the Bayesian restoration of images', IEEE Transactions on Pattern Analysis and Machine Intelligence, Vol. PAMI-6, No. 6, pp721-741.

Geweke, J. (1992) 'Evaluating the accuracy of sampling-based approaches to the calculation of posterior moments', In Bayesian Statistics 4, Bernardo, J.M., Berger, J.O., Dawid, A. P., and Smith, A.F.M. (eds.), pp169-193. Oxford: Oxford University Press.

Gill, J. (2007) Bayesian Methods: A Social and Behavioral Sciences Approach, 2nd Edition, Chapman and Hall/CRC.

Goodman, L.A. (1974) 'Exploratory latent structure analysis using both identifiable and unidentifiable models', Biometrika, Vol.61, No. 2, pp215-231.

Guttman, I. (1967) 'The use of the concept of a future observation in goodness-of-fit problems', Journal of the Royal Statistical Society B, Vol. 29, No. 1, pp.83-100.

Haberman, S.J. (1974) 'Log-linear model for frequency tables derived by indirect observation: maximum likelihood equations', Annals of Statistics, Vol. 2, No. 5, pp911-924.

Haberman, S.J. (1979) Qualitative data analysis, Vol. 2, New developments, New York: Academic Press.

Haertel, E.H. (1989) 'Using restricted latent class models to map the skill structure of achievement items', Journal of Educational Measurement, Vol. 26, No. 4, pp.333-352.

Hambleton, R.K. and Han, N. (2004) 'Assessing the fit of IRT models: some approaches 
and graphical displays', Paper presented at the Annual Meeting of the National Council on Measurement in Education, San Diego.

Hartz, S. (2002) A Bayesian Framework for the Unified Model for Assessing Cognitive Abilities: Blending Theory with Practice, Unpublished doctoral thesis, University of Illinois at Urbana-Champain.

Hastings, W.K. (1970) 'Monte Carlo sampling methods using Markov chains, and their applications', Biometrika, Vol. 57, No. 1, pp97-109.

Henson, R., Templin, J. and Douglas, J. (2007) 'Using efficient model based sum-scores for conducting skill diagnoses', Journal of Educational Measurement, Vol. 44, No. 4, pp.361-376.

Hoijtink, H.(2001) 'Conditional independence and differential item functioning in the two-parameter logistic model', in A. Boomsma, M.A.J. van Duijn and T.A.B. Holland, P. W. (1985) 'On the study of differential item performance without IRT' .Proceedings of the $27^{\text {th }}$ annual conference of the Military Testing Association. Vol.I, 282-287. San Diego.

Snijders (Eds.): Essays in Item Response Theory, pp.109-130, Springer-Verlag, New York, NY.

Jannsen, R., Tuerlinckx, F., Meulders, M. and De Boeck, P. (2000) 'A hierarchical IRT model for criterion-referenced measurement', Journal ofEducational and Behavioral Statistics, Vol. 25, No. 3, pp.285-306.

Johnson, M.S. and Junker, B.W. (2003) 'Using data augmentation and Markov chain Monte Carlo for the estimation of unfolding response models', Journal of 
Educational and Behavioral Statistics, Vol. 28, No. 3, pp195-230.

Johnson, M. S. (2007) 'Marginal Maximum Likelihood Estimation of Item Response Models in R', Journal of Statistical Software. Vol. 20, No.10.

Junker, B.W. and Sijtsma, K. (2001) 'Cognitive assessment models with few assumptions, and connections with nonparametric item response theory', Applied Psychological Measurement, Vol. 25, No. 3, pp.258-272.

Lazarsfeld, P.F. and Henry, N.W. (1968) Latent structure analysis, Boston: Houghton Mifflin.

Levy, R. (2006) Posterior Predictive Model Checking for Multidimensionality in Item Response Theory and Bayesian Networks, Unpublished doctoral dissertation, University of Maryland.

Levy, R. (2011) 'Posterior predictive model checking for conjunctive multidimensionality in item response theory', Journal of Educational and Behavioral Statistics, Vol. 36, No. 5, pp.672-694.

Levy, R., Mislevy, R.J. and Sinharay, S. (2009) 'Posterior predictive model checking for multidimensionality in item response theory', Applied Psychological Measurement, Vol. 33, No. 7, pp.519-537.

Liebetrau, A.M. (1983) ‘Measures of association’, Newbury Park, CA:Sage.

Liu, J., Xu, G., and Ying, Z. (2012) 'Data-driven learning of Q-matrix', Applied Psychological Measurement, Vol. 36, No. 7, pp609-618.

Lunn, D., Spiegelhalter, D., Thomas, A. and Best, N. (2009) 'The BUGS project: evolution, critique and future directions (with discussion)', Statistics in Medicine, 
Vol. 28, No. 25, pp.3049-3082.

Macready, G.B. and Dayton, C.M. (1977) 'The use of probabilistic models in the assessment of mastery', Journal of Educational Statistics, Vol. 2, No. 2, pp.99120.

Maris, E. (1995) 'Psychometric latent response models', Psychometrika, Vol. 60, No. 4, pp. 523-547.

Maris, E. (1999) 'Estimating multiple classification latent class models', Psychometrika, Vol. 64, No. 2, pp.187-212.

Martyn, P., Nicky, B., Kate, C., and Karen, V. (2006) CODA: Convergence Diagnosis and Output Analysis for MCMC, R News, Vol 6, pp.7-11

Meng, X.L. (1994) 'Posterior predictive p-values', The Annals of Statistics, Vol. 22, No. 3, pp.1142-1160.

Metropolis, N., Rosenbluth, A.W., Rosenbluth, M.N., Teller, A.H., and Teller, E. (1953) 'Equations of state calculations by fast computing machines', Journal of Chemical Physics, Vol. 21, No. 6, pp1087-1091.

Neal, R.M. (2003) 'Slice sampling', The Annals of Statistics, Vol. 31, No. 3, pp 705-741. Neyman, J. and Scott, E.L. (1948) 'Consistent estimation from partially consistent observations', Econometrica, Vol. 16, pp.1-32.

Park, J.Y., Johnson, M.S., and Lee, Y.S. (2015) 'Posterior Predictive Model Checks for Cognitive Diagnostic Models', International Journal of Quantitative Research in Education, Vol. 2, No. 3-4, pp244-264.

Patz, R.J. and Junker, B.W. (1999) 'A straightforward approach to Markov chain Monte 
Carlo methods for item response theory models', Journal of Educational and Behavioral Statistics, Vol. 24, No. 2, pp146-178.

Rasch, G. (1960 [1980]) Probabilistic models for some intelligence and attainment tests, Chicago: University of Chicago Press.

R Core Team (2013) R: A Language and Environment for Statistical Computing, $\mathrm{R}$ Foundation for Statistical Computing, Vienna, Austria [online] http://www.Rproject.org/.

Rho, Y. J. (2010) Cognitive Skill Diagnosis in the Presence of Differential Strategy Choice: A Bayesian Approach, Unpublished doctoral dissertation, Columbia University. Robin, J.M., van der Vaart, A., and Ventura, V. (2000) 'The asymptotic distribution of p values in composite null models', Journal of the American Statistical Association, Vo. 95, No. 452, pp.1143-1172.

Robitzsch, A., Kiefer, T., George, A.C. and Uenlue, A. (2013) CDM: Cognitive Diagnosis Modeling, R Package Version 2.4-9 [online] http://CRAN.R project.org/package $=\mathrm{CDM}$.

Rubin, D.B. (1984) 'Bayesianly justifiable and relevant frequency calculations for the applied statistician', Annals of statistics, Vol. 12, No. 4, pp.1151-1172.

Rupp, A.A. and Templin, J. (2008) 'The effect of Q-matrix misspecification on parameter estimates and classification accuracy in the DINA model', Educational and Psychological Measurement, Vol. 68, No. 1, pp.78-96.

Rupp, A.A., Templin, J., and Henson, R.A. (2010) Diagnostic measurement: theory, methods, and applications, Guilford. 
Schwarz, G.E. (1978) 'Estimating the dimensions of a model', Annals of Statistics, Vol. 6, No. 2, pp. 461-464.

Sinharay, S. (2005) 'Assessing fit of unidimensional item response theory models using a Bayesian approach', Journal of Educational Measurement, Vol. 42, No. 4, pp.375-394.

Sinharay, S. (2006) 'Model diagnostics for Bayesian network', Journal of Educational and Behavioral Statistics, Vol. 31, No. 1, pp.1-33.

Sinharay, S. and Almond, R.G. (2007) 'Assessing fit of cognitive diagnostic models: a case study', Educational and Psychological Measurement, Vol. 67, No. 2, pp.239-257.

Sinharay, S., Johnson, M.S. and Stern, H.S. (2006) 'Posterior predictive assessment of item response theory models', Applied Psychological Measurement, Vol. 30, No. 4, pp.298-321.

Spiegelhalter, D. J, Best, N. G., Carlin, B. P., and van der Linde, A. (2002) 'Bayesian measures of model complexity and fit', Journal of the Royal Statistical Society, Series B, Methodological, Vol. 64, No. 4, pp.1-34.

Sturtz, S., Ligges, U. and Gelman, A. (2005) 'R2WinBUGS: a package for running WinBUGS from R', Journal of Statistical Software, Vol. 12, No. 3, pp.1-16.

Tatsuoka, C. (2002) 'Data analytic methods for latent partially ordered classification models', Journal of the Royal Statistical Society: Series C (Applied Statistics), Vol. 51, No. 3, pp.337-350.

Tatsuoka, K.K. (1985) 'A probabilistic model for diagnosing misconceptions in the 
pattern classification approach', Journal of Educational Statistics, Vol. 10, No. 1, pp.55-73.

Tatsuoka, K.K. (1990) 'Toward an integration of item-response theory and cognitive error diagnosis', in N. Frederiksen, R. Glaser, A. Lesgold and M. Shafto (Eds.): Diagnostic Monitoring of Skill and Knowledge Acquisition, pp.453-488, Erlbaum, Mahwah, NJ.

Templin, J.L. and Henson, R.A. (2006) 'Measurement of psychological disorders using cognitive diagnosis models', Psychological Methods, Vol. 11, No. 3, pp.287-305.

Tierney, L. (1994) 'Markov chains for exploring posterior distributions (with discussion)', Annals of Statistics, Vol. 22, No. 4, pp1701-1762.

Tseng, H. (2010) A Linear Compensatory Counterpart to and Generalization of the DINA Model, Unpublished doctoral dissertation, Columbia University.

von Davier, M (2014) 'The DINA model as a constrained general diagnostic model: two variants of a model equivalency', British Journal of Mathematical and Statistical Psychology, Vol. 67, No. 1, pp.49-71.

von Davier, M. (2005) A General Diagnostic Model Applied to Language Testing Data, Research Report RR-05-16, ETS, Princeton, NJ.

von Davier, M. and Yamamoto, K. (2004a) 'A class of models for cognitive diagnosis', Paper presented at the 4th Spearman Conference, Philadelphia, PA, October. von Davier, M. and Yamamoto, K. (2004b) 'A class of models for cognitive diagnosis and some notes on estimation', Paper presented at the ETS Tucker Workshop Seminar, Princeton, NJ, December. 
Wang, C., Shu, Z., Shang, Z., and Xu, G. (2015) 'Assessing item-level fit for the DINA model', Applied Psychological Measurement.

Wu, H., Yuen, K. and Leung, S. (2014) 'A novel relative entropy-posterior predictive model checking approach with limited information statistics for latent trait models in sparse $2^{\mathrm{k}}$ contingency table', Computational Statistics and Data Analysis, Vol. 79, pp.261-276.

Xiang, R. (2013) 'Nonlinear penalized estimation of true Q-matrix in cognitive diagnostic models', Columbia University (Ph.D dissertation).

$\mathrm{Xu}, \mathrm{X}$. and von Davier, M. (2008a) 'Fitting the structured general diagnostic model to NAEP data', Education Testing Service. 


\section{Appendix A. R20penBUGS code}

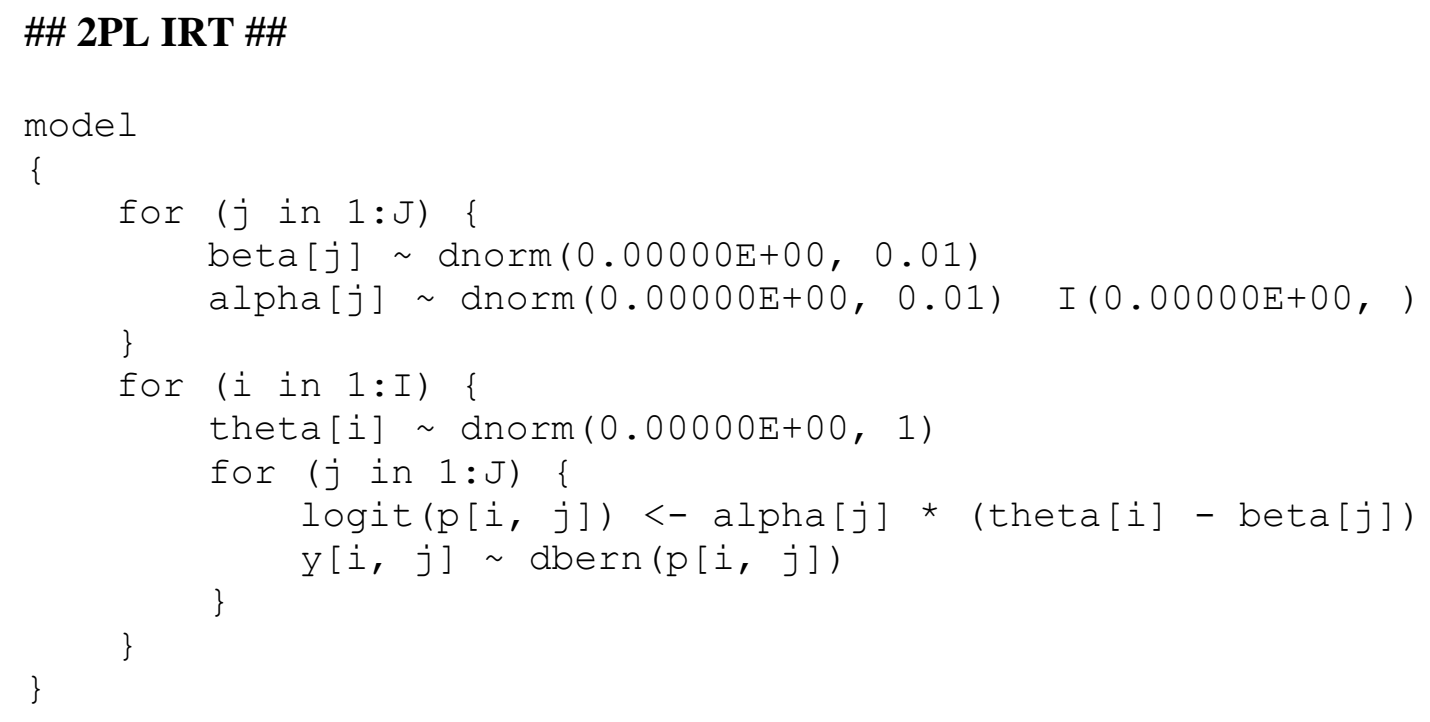




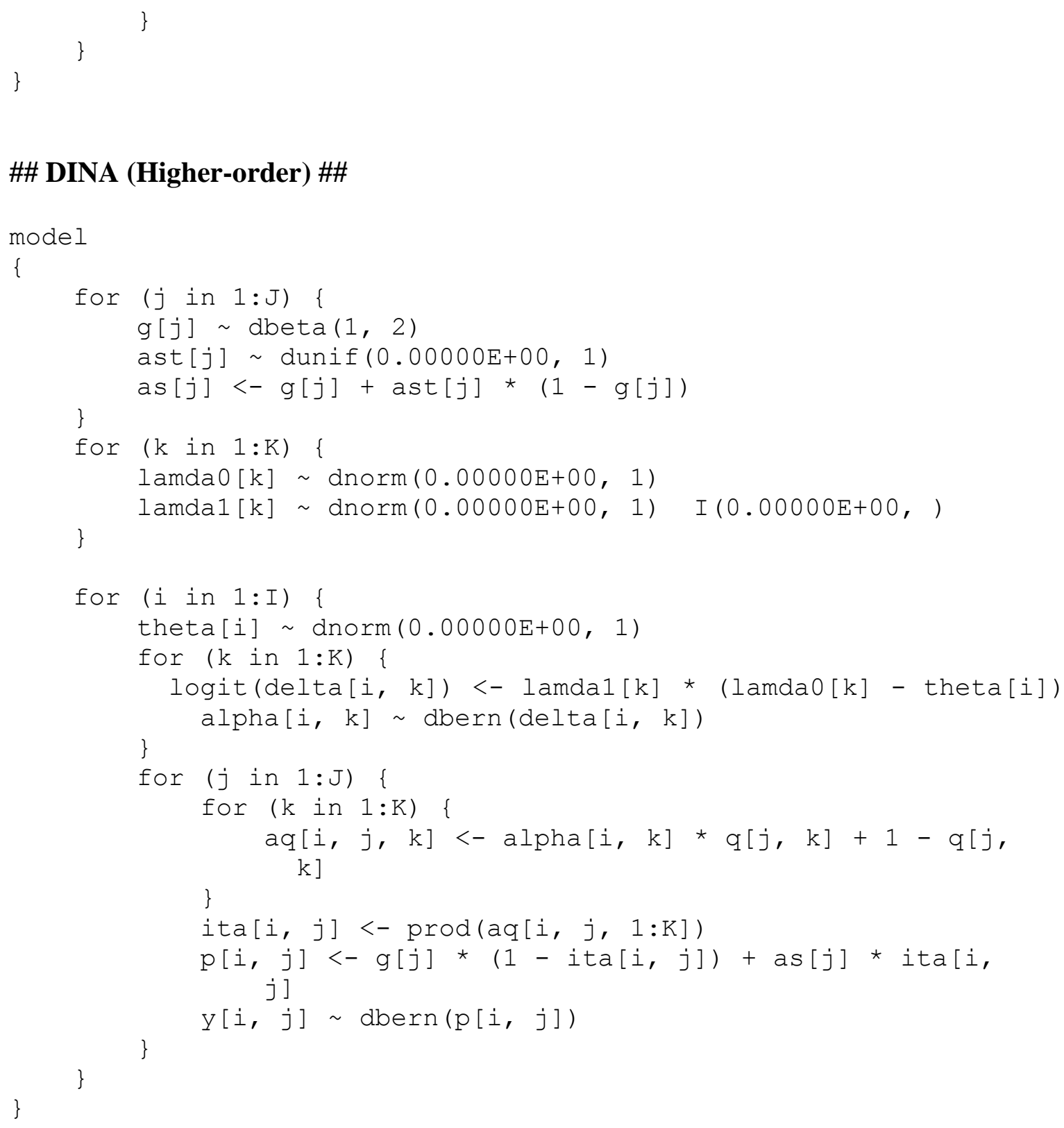

\section{\#\# DINA (Saturated) \#\#}

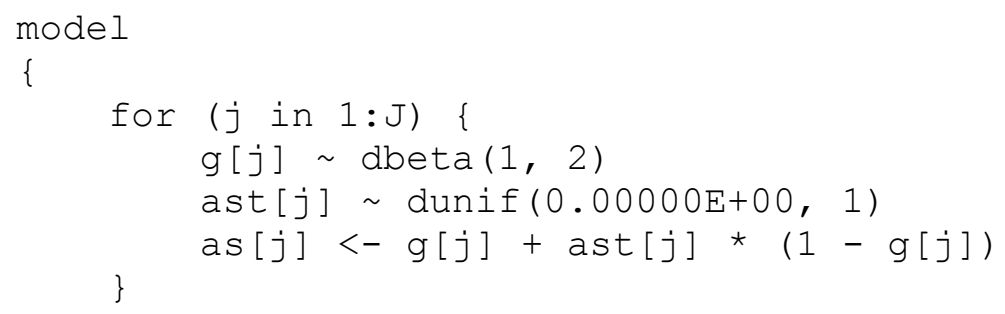




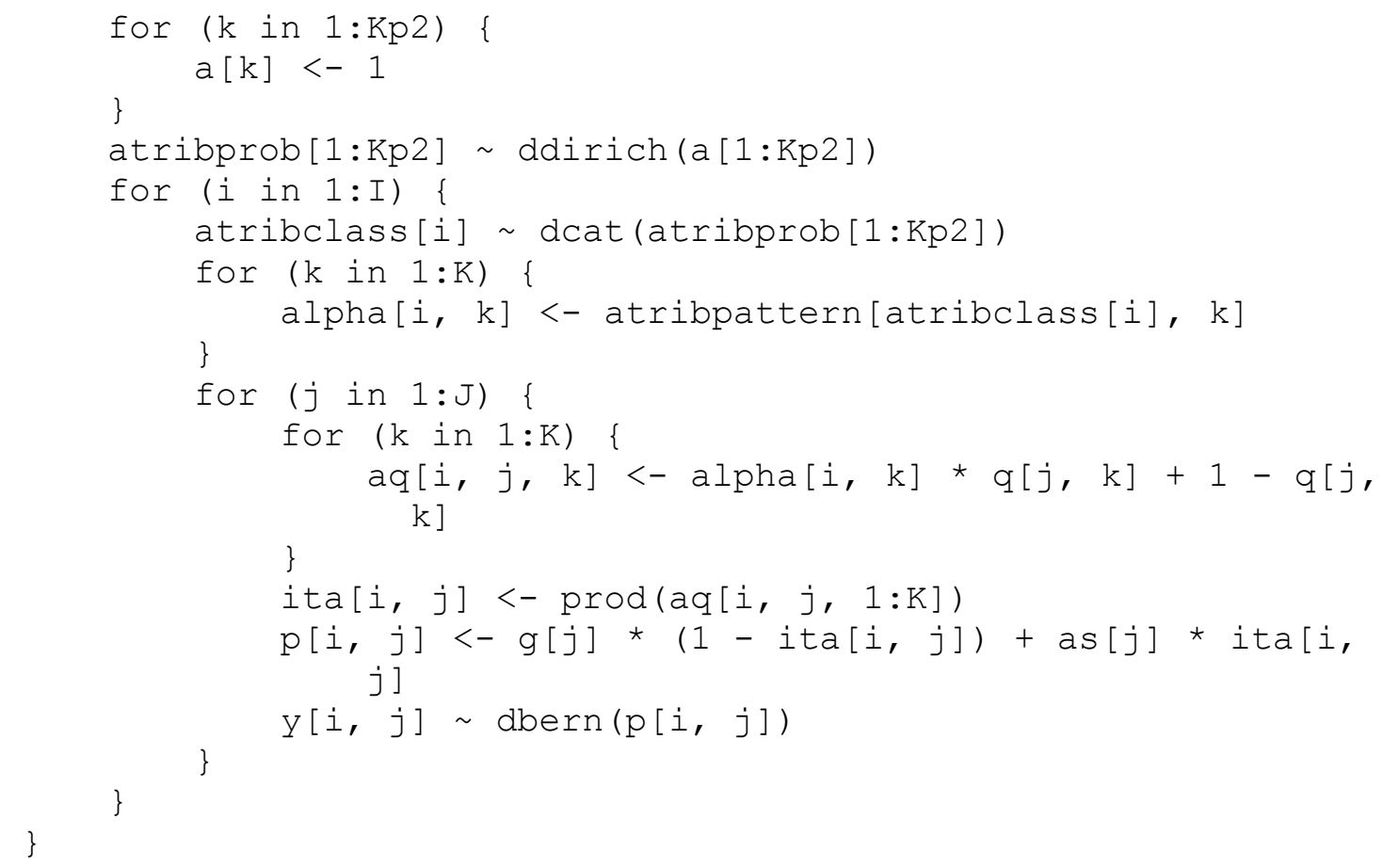

\section{\#\# GDM (Constant Slopes) \#\#}

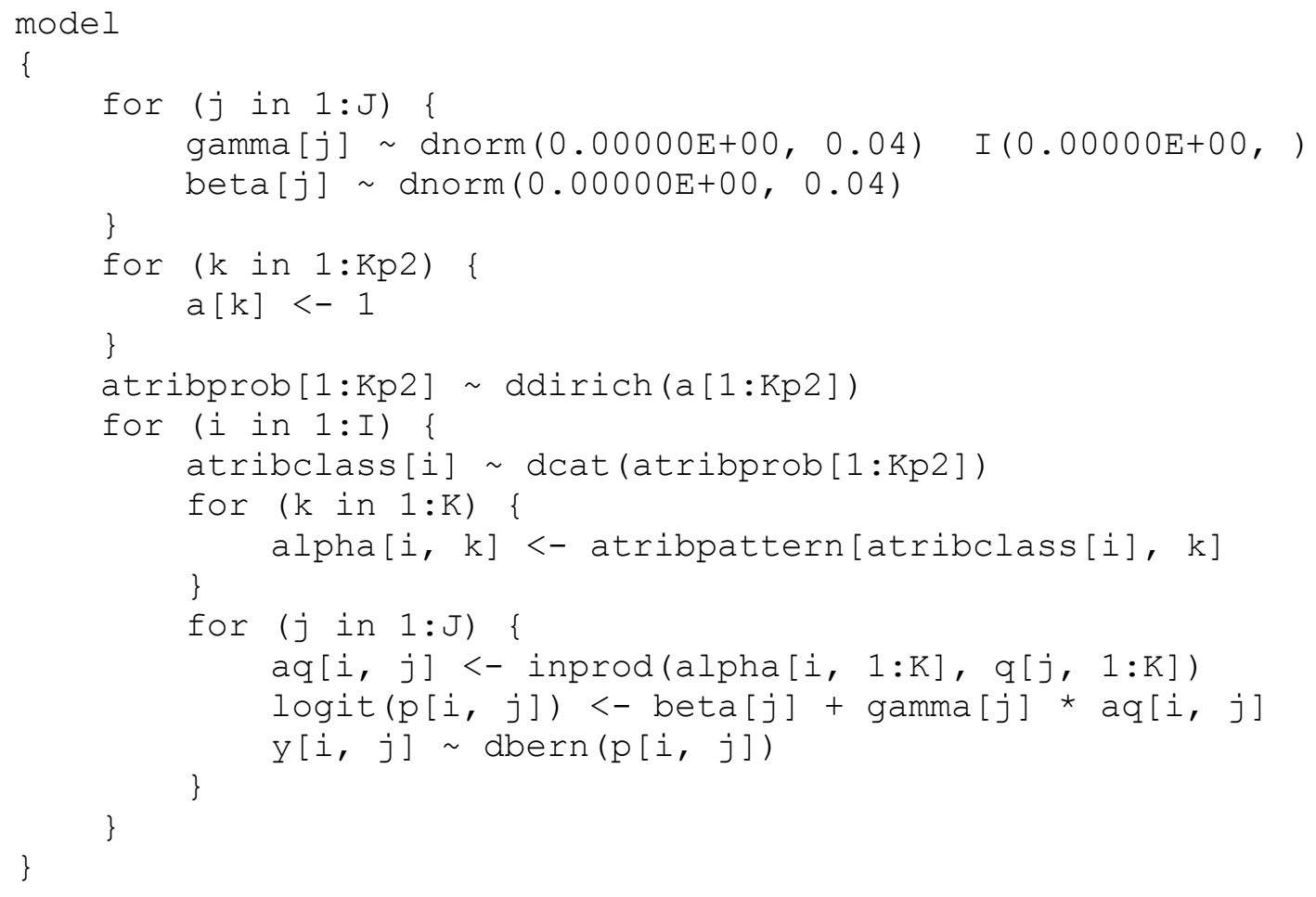




\section{\#\# GDM (Varying Slopes) \#\#}

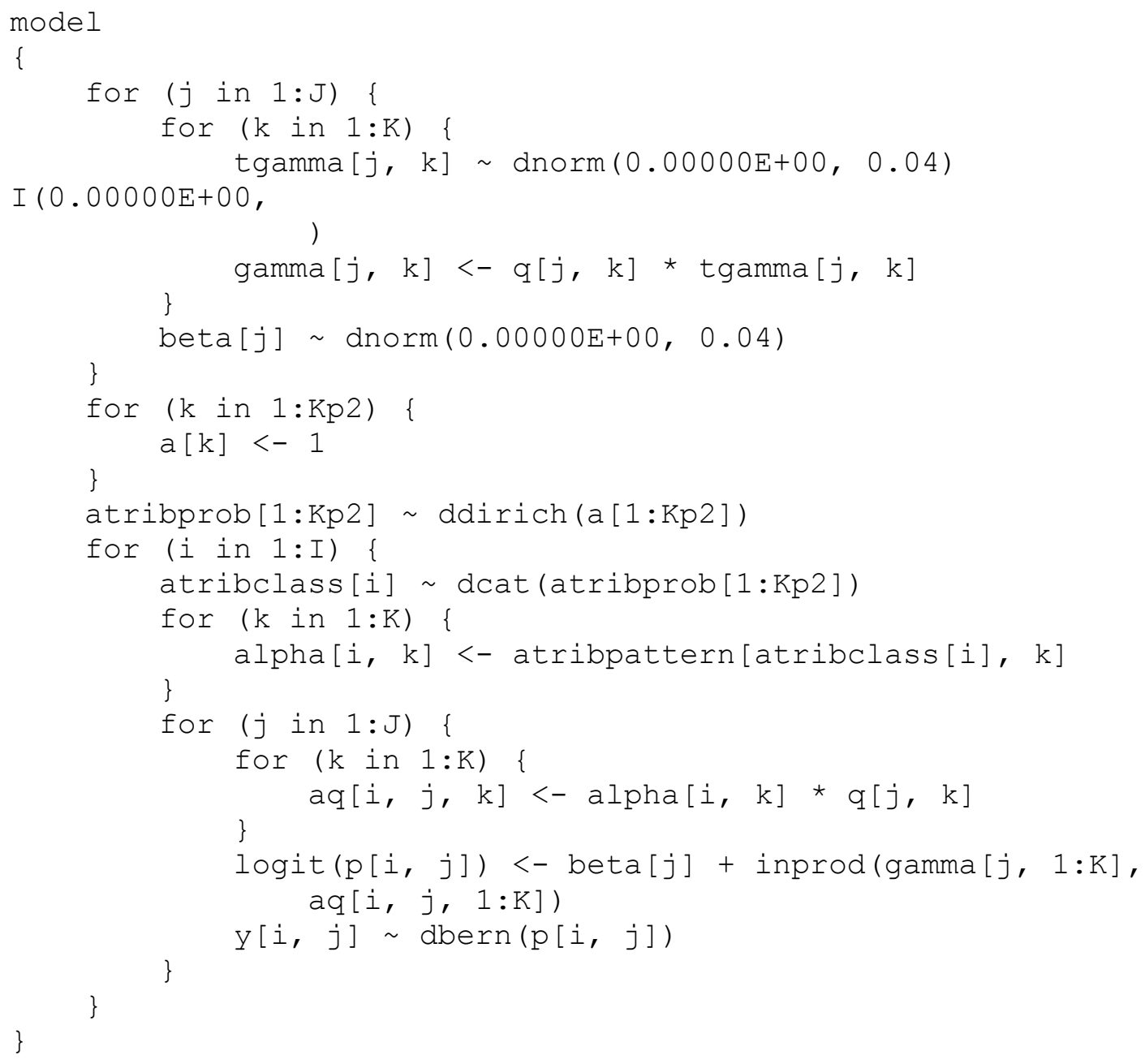




\title{
Appendix B. R code for Posterior Predictive Model Checks
}

\author{
\#\# 2PL IRT \#\#
}

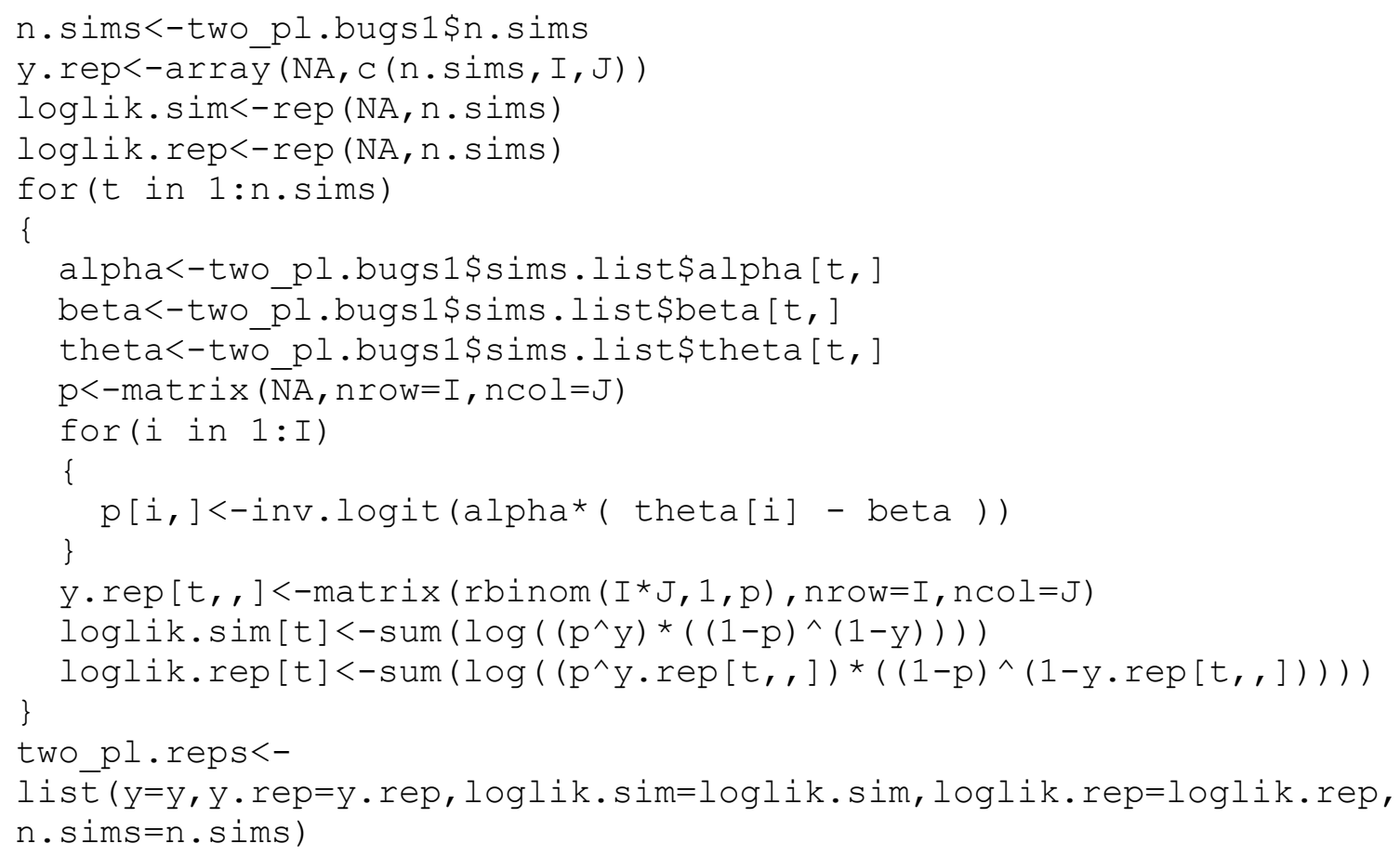

\section{\#\# DINA (Independence) \#\#}

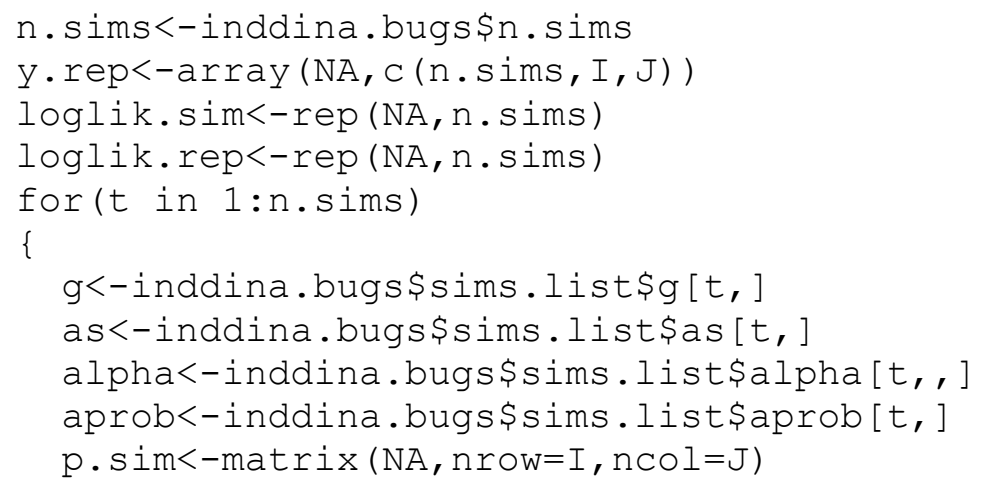




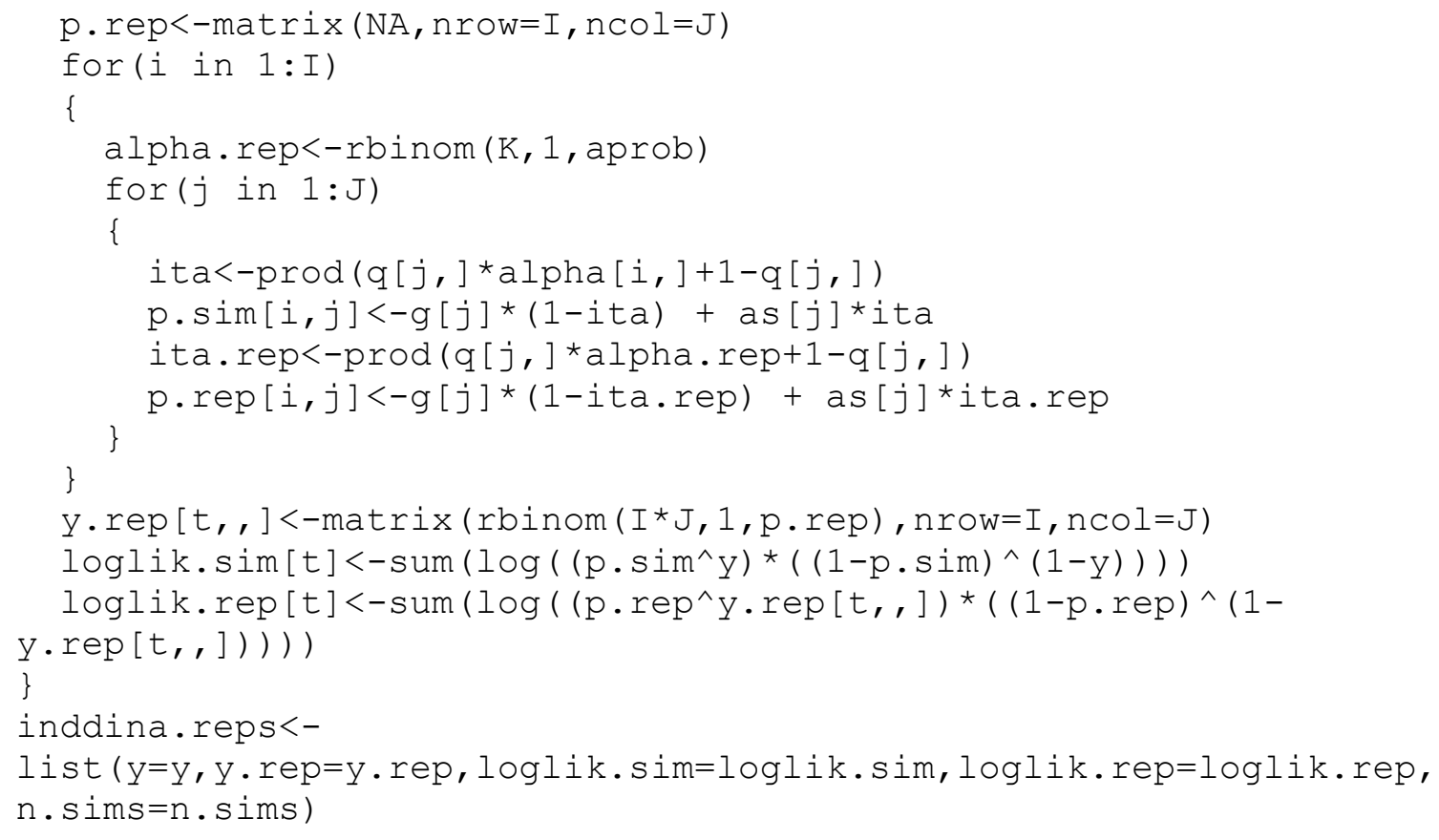

\section{\#\# DINA (Higher-order) \#\#}

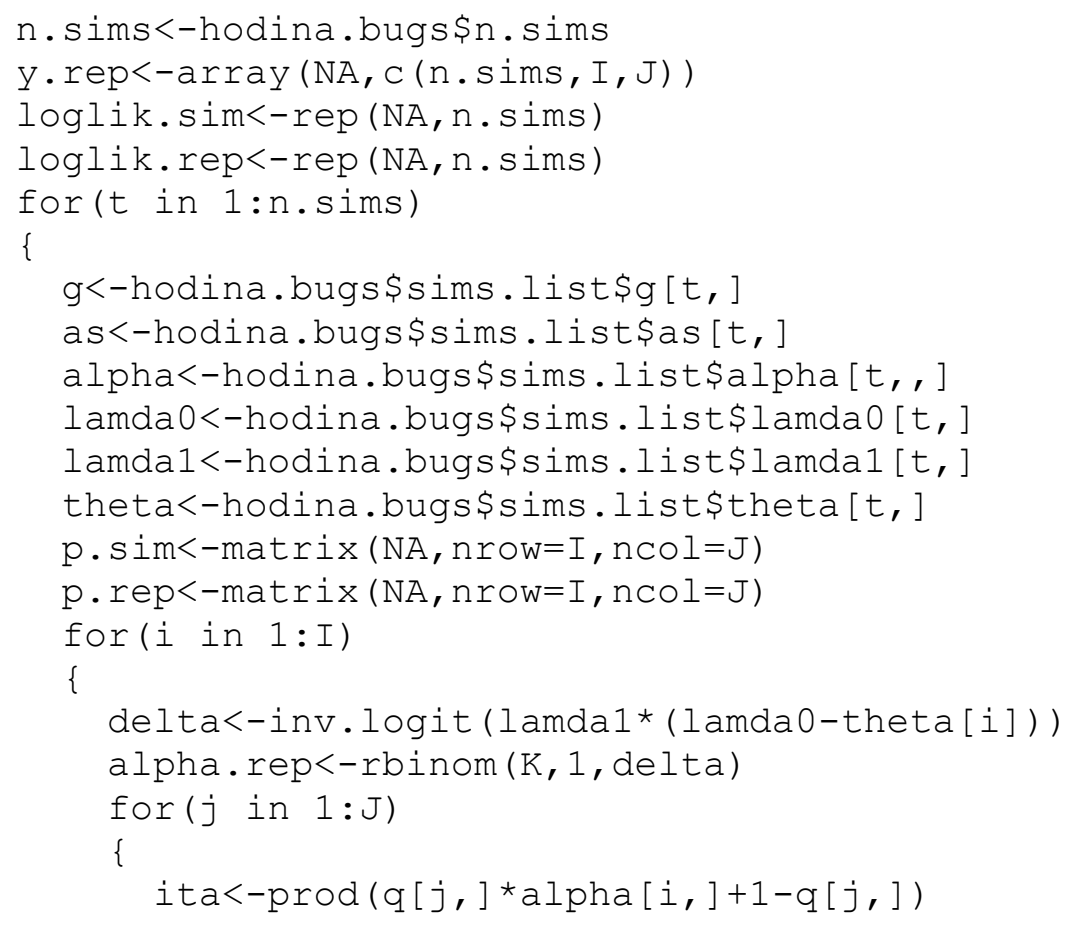




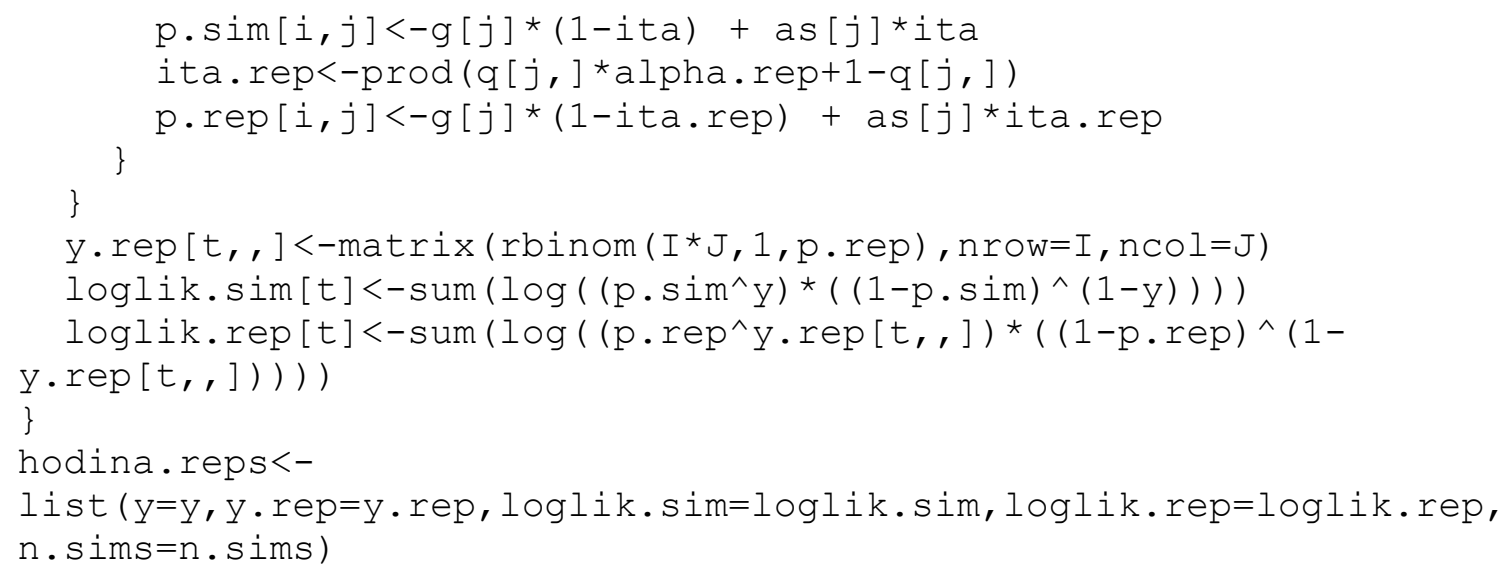

\section{\#\# DINA (Saturated) \#\#}

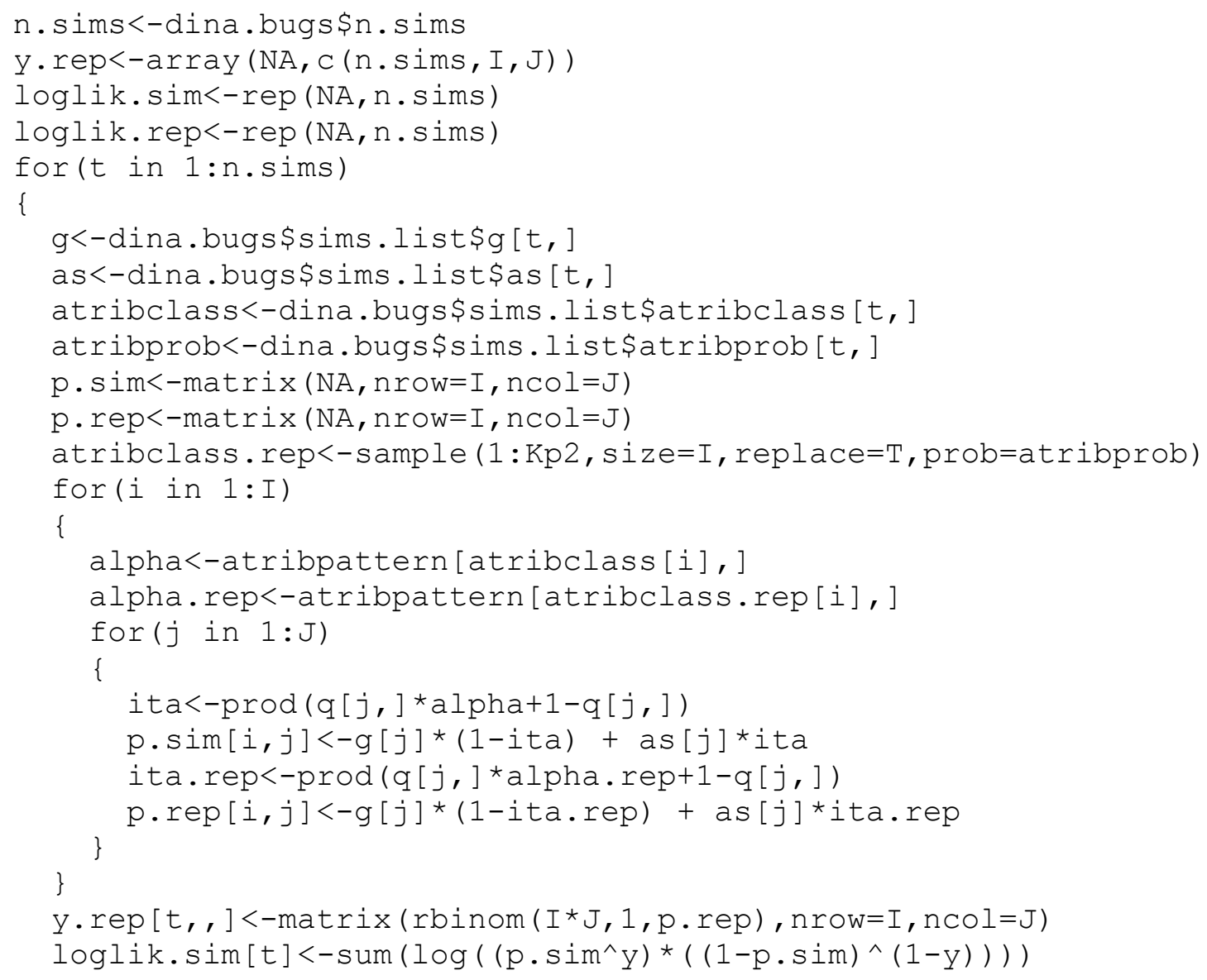




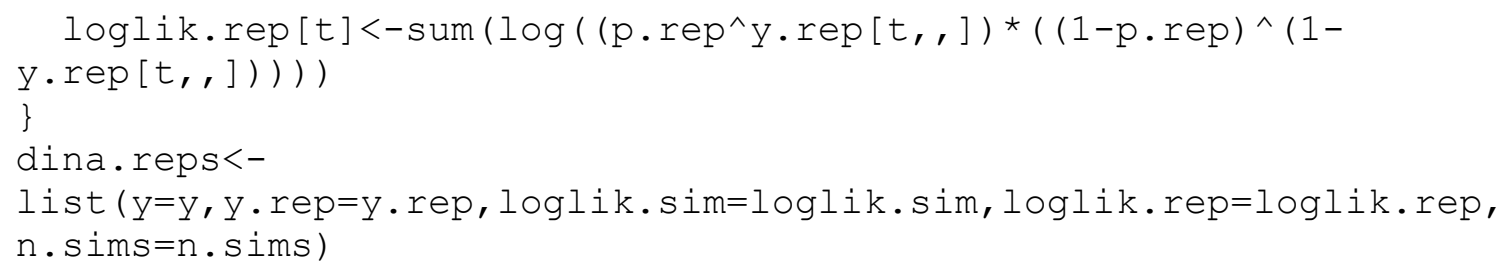

\section{\#\# GDM (Constant Slopes) \#\#}

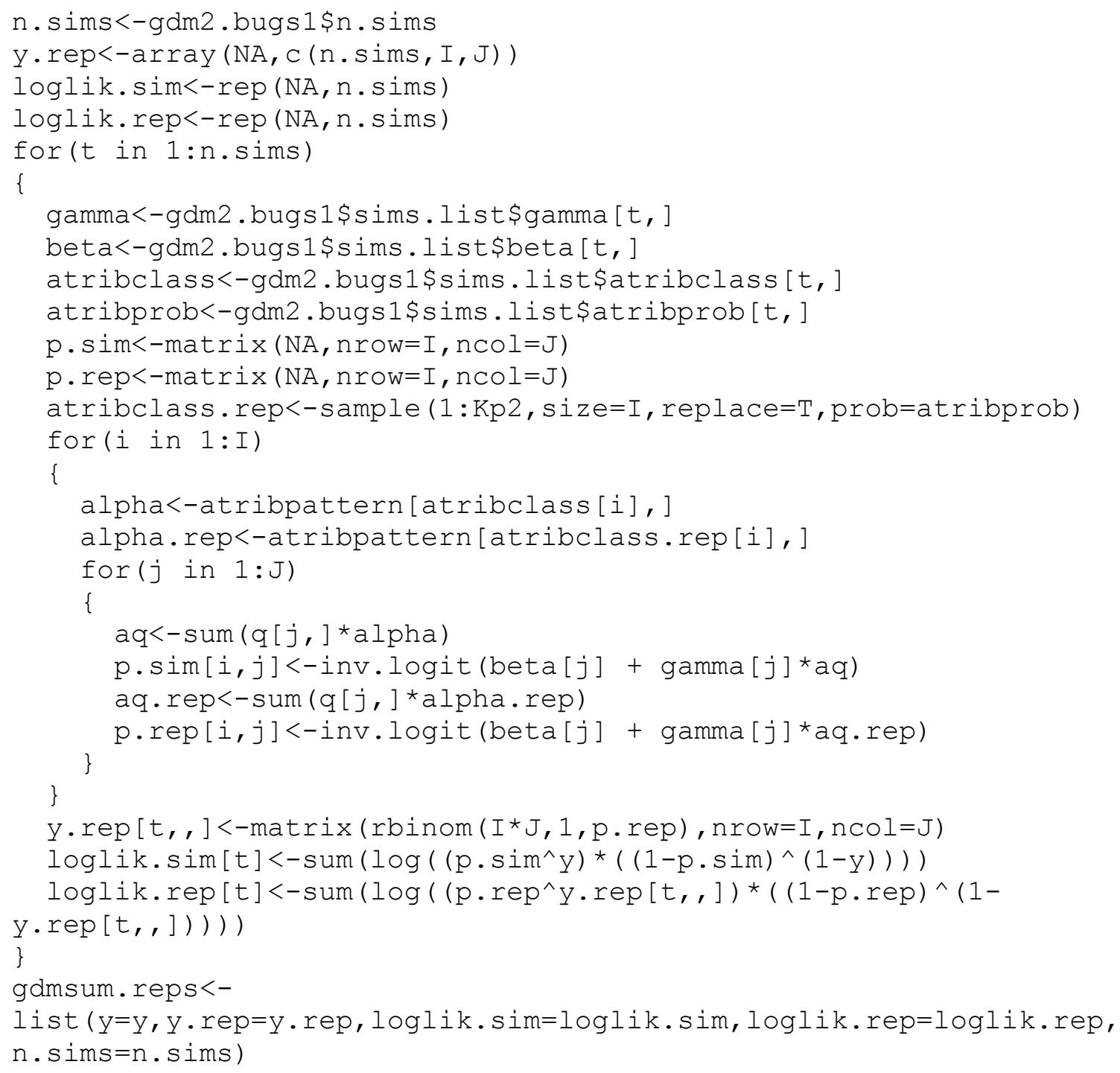




\section{\#\# GDM (Varying Slopes) \#\#}

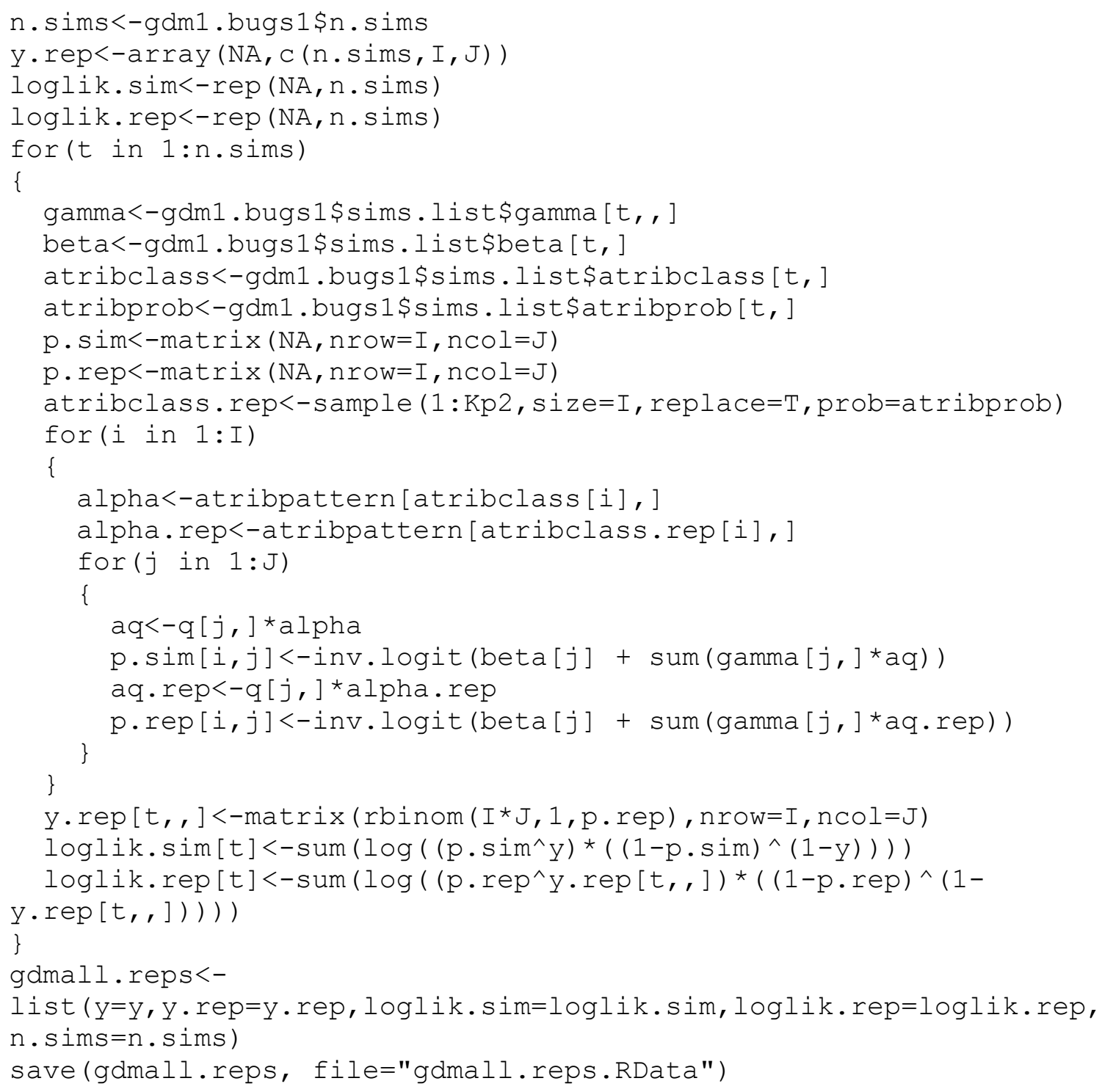




\section{Appendix C. Convergence Diagnostics}

\section{PL IRT Model}

Table C.1. Geweke Statistics for Intercept and Location Parameters

\begin{tabular}{ccccclcccc}
\hline Parameter & Chain1 & Chain2 & Chain3 & Chain4 & Parameter & Chain1 & Chain2 & Chain3 & Chain4 \\
\hline beta[1] & 0.16 & -1.23 & 1.29 & 1.55 & gamma[1] & -2.22 & 2.38 & 0.57 & 0.28 \\
beta[2] & -0.52 & -0.88 & 0.35 & 0.16 & gamma[2] & -0.84 & 0.23 & -0.23 & -0.28 \\
beta[3] & 0.22 & 0.37 & -0.94 & 1.14 & gamma[3] & -0.32 & 0.27 & -1.24 & 1.05 \\
beta[4] & 1.15 & -0.97 & 0.33 & -0.24 & gamma[4] & -0.68 & 0.42 & -0.09 & 1.05 \\
beta[5] & 1.24 & -0.75 & 0.50 & 2.92 & gamma[5] & 1.55 & -0.08 & 1.60 & 1.32 \\
beta[6] & 1.21 & 0.38 & 0.64 & 1.28 & gamma[6] & -1.08 & -0.80 & 0.71 & -0.56 \\
beta[7] & 0.68 & -1.12 & 1.25 & 1.62 & gamma[7] & -0.06 & 0.69 & -1.34 & -2.11 \\
beta[8] & 0.96 & -0.78 & 0.41 & -0.13 & gamma[8] & -0.30 & 0.11 & 0.54 & -0.10 \\
beta[9] & 0.97 & -1.02 & 0.99 & -0.04 & gamma[9] & 0.28 & 0.50 & 1.21 & -0.57 \\
beta[10] & 1.21 & -1.07 & 0.64 & -0.04 & gamma[10] & -0.59 & 1.65 & 0.11 & -0.19 \\
beta[11] & 1.02 & 0.37 & 0.61 & 0.26 & gamma[11] & -0.97 & 0.42 & -0.27 & -1.46 \\
beta[12] & -0.10 & -0.33 & 0.84 & -0.04 & gamma[12] & 0.67 & 0.80 & 0.41 & 1.30 \\
beta[13] & 0.32 & -0.77 & 0.41 & 0.91 & gamma[13] & -1.03 & -0.08 & -0.57 & 0.67 \\
beta[14] & 1.89 & -1.06 & 0.63 & 2.22 & gamma[14] & -2.18 & 1.64 & 0.42 & -2.56 \\
beta[15] & 0.72 & -0.81 & 1.05 & -0.15 & gamma[15] & -0.46 & -0.24 & -0.40 & 0.74 \\
\hline
\end{tabular}

Table C.2. Gelman and Rubin Statistics for Intercept and Location Parameters

\begin{tabular}{cccccc}
\hline Parameter & Est. & $\begin{array}{c}\text { Upper } \\
\text { CI }\end{array}$ & Parameter & Est. & $\begin{array}{c}\text { Upper } \\
\text { CI }\end{array}$ \\
\hline beta[1] & 1.00 & 1.00 & gamma[1] & 1.00 & 1.00 \\
beta[2] & 1.00 & 1.00 & gamma[2] & 1.00 & 1.00 \\
beta[3] & 1.00 & 1.00 & gamma[3] & 1.00 & 1.00 \\
beta[4] & 1.00 & 1.01 & gamma[4] & 1.00 & 1.00 \\
beta[5] & 1.00 & 1.00 & gamma[5] & 1.00 & 1.00 \\
beta[6] & 1.00 & 1.00 & gamma[6] & 1.00 & 1.00 \\
beta[7] & 1.00 & 1.00 & gamma[7] & 1.00 & 1.00 \\
beta[8] & 1.00 & 1.00 & gamma[8] & 1.00 & 1.00 \\
beta[9] & 1.00 & 1.00 & gamma[9] & 1.00 & 1.00 \\
beta[10] & 1.00 & 1.00 & gamma[10] & 1.00 & 1.00 \\
beta[11] & 1.00 & 1.00 & gamma[11] & 1.00 & 1.00 \\
beta[12] & 1.00 & 1.00 & gamma[12] & 1.00 & 1.00 \\
beta[13] & 1.00 & 1.00 & gamma[13] & 1.00 & 1.00 \\
beta[14] & 1.00 & 1.01 & gamma[14] & 1.00 & 1.00 \\
beta[15] & 1.00 & 1.00 & gamma[15] & 1.00 & 1.00 \\
\hline
\end{tabular}




\section{DINA with Independence structure}

Table C.3. Geweke Statistics for Anti-slip and Guessing Parameters

\begin{tabular}{cccccccccc}
\hline Parameter & Chain1 & Chain2 & Chain3 & Chain4 & Parameter & Chain1 & Chain2 & Chain3 & Chain4 \\
\hline as[1] & 1.75 & 0.80 & -1.20 & 0.52 & $\mathrm{~g}[1]$ & 1.31 & 0.20 & -2.12 & 0.60 \\
as[2] & 0.21 & 0.11 & -0.04 & -0.64 & $\mathrm{~g}[2]$ & -0.30 & 1.07 & 0.39 & 0.16 \\
as[3] & 0.21 & 0.75 & -0.46 & -0.66 & $\mathrm{~g}[3]$ & 2.51 & -0.18 & -0.75 & 0.61 \\
as[4] & 1.39 & -0.07 & -0.12 & -0.49 & $\mathrm{~g}[4]$ & 0.26 & -1.54 & 0.71 & 0.28 \\
as[5] & -0.21 & -0.47 & 1.06 & -0.62 & $\mathrm{~g}[5]$ & -0.89 & 0.35 & 2.73 & -1.82 \\
as[6] & 1.92 & -0.65 & -0.20 & -0.11 & $\mathrm{~g}[6]$ & -2.06 & 1.46 & -0.39 & 0.01 \\
as[7] & -0.69 & -1.32 & 0.20 & 0.31 & $\mathrm{~g}[7]$ & -1.25 & 2.45 & 1.73 & -0.21 \\
as[8] & -0.43 & 1.26 & -2.28 & -4.64 & $\mathrm{~g}[8]$ & 1.15 & -0.32 & 0.08 & 0.22 \\
as[9] & -1.07 & 0.34 & 1.10 & 0.16 & $\mathrm{~g}[9]$ & 0.65 & 0.44 & -0.09 & -1.00 \\
as[10] & -1.61 & 0.42 & -0.21 & -0.33 & $\mathrm{~g}[10]$ & 0.28 & -0.01 & 0.22 & -1.92 \\
as[11] & 0.49 & 1.12 & 0.36 & -2.38 & $\mathrm{~g}[11]$ & 0.29 & 0.49 & 0.88 & -0.90 \\
as[12] & 0.11 & 0.08 & 0.69 & 0.35 & $\mathrm{~g}[12]$ & -2.07 & 0.54 & 0.04 & 0.84 \\
as[13] & -0.71 & -1.03 & 1.55 & -1.79 & $\mathrm{~g}[13]$ & -0.55 & 0.36 & 2.93 & -3.69 \\
as[14] & 0.50 & 0.97 & 0.87 & -0.47 & $\mathrm{~g}[14]$ & 0.44 & 0.56 & 0.90 & 0.78 \\
as[15] & -0.43 & 0.74 & 0.91 & 0.61 & $\mathrm{~g}[15]$ & 0.15 & 0.44 & -0.76 & 0.14 \\
\hline
\end{tabular}

Table C.4. Gelman and Rubin Statistics for Anti-slip and Guessing Parameters

\begin{tabular}{cccccc}
\hline Parameter & Est. & $\begin{array}{c}\text { Upper } \\
\text { CI }\end{array}$ & Parameter & Est. & $\begin{array}{c}\text { Upper } \\
\text { CI }\end{array}$ \\
\hline as[1] & 1.00 & 1.01 & $\mathrm{~g}[1]$ & 1.00 & 1.00 \\
$\operatorname{as}[2]$ & 1.00 & 1.00 & $\mathrm{~g}[2]$ & 1.00 & 1.00 \\
$\operatorname{as}[3]$ & 1.00 & 1.00 & $\mathrm{~g}[3]$ & 1.00 & 1.01 \\
$\operatorname{as}[4]$ & 1.00 & 1.00 & $\mathrm{~g}[4]$ & 1.00 & 1.00 \\
$\operatorname{as}[5]$ & 1.00 & 1.00 & $\mathrm{~g}[5]$ & 1.00 & 1.00 \\
$\operatorname{as}[6]$ & 1.00 & 1.00 & $\mathrm{~g}[6]$ & 1.00 & 1.00 \\
$\operatorname{as}[7]$ & 1.00 & 1.00 & $\mathrm{~g}[7]$ & 1.00 & 1.00 \\
$\operatorname{as}[8]$ & 1.00 & 1.00 & $\mathrm{~g}[8]$ & 1.00 & 1.01 \\
$\operatorname{as}[9]$ & 1.00 & 1.00 & $\mathrm{~g}[9]$ & 1.00 & 1.00 \\
$\operatorname{as}[10]$ & 1.00 & 1.00 & $\mathrm{~g}[10]$ & 1.00 & 1.00 \\
$\operatorname{as}[11]$ & 1.00 & 1.00 & $\mathrm{~g}[11]$ & 1.00 & 1.00 \\
$\operatorname{as}[12]$ & 1.00 & 1.00 & $\mathrm{~g}[12]$ & 1.00 & 1.00 \\
$\operatorname{as}[13]$ & 1.00 & 1.00 & $\mathrm{~g}[13]$ & 1.00 & 1.00 \\
$\operatorname{as}[14]$ & 1.00 & 1.01 & $\mathrm{~g}[14]$ & 1.00 & 1.00 \\
$\operatorname{as}[15]$ & 1.00 & 1.00 & $\mathrm{~g}[15]$ & 1.00 & 1.00 \\
\hline
\end{tabular}




\section{DINA with Higher-order structure}

Table C.5. Geweke Statistics for Anti-slip and Guessing Parameters

\begin{tabular}{cccccccccc}
\hline Parameter & Chain1 & Chain2 & Chain3 & Chain4 & Parameter & Chain1 & Chain2 & Chain3 & Chain4 \\
\hline as[1] & -0.25 & -0.94 & -1.09 & 0.03 & $\mathrm{~g}[1]$ & 1.22 & 0.40 & -0.44 & -0.42 \\
as[2] & 0.81 & 0.88 & 0.03 & 0.88 & $\mathrm{~g}[2]$ & 0.81 & 1.20 & -0.46 & 0.52 \\
as[3] & -2.04 & -1.06 & 2.06 & 1.13 & $\mathrm{~g}[3]$ & -1.26 & -2.01 & -0.70 & -0.33 \\
as[4] & 1.99 & -1.18 & -1.40 & 1.34 & $\mathrm{~g}[4]$ & 2.79 & -0.64 & -0.39 & 1.35 \\
as[5] & 0.13 & 0.05 & -0.40 & 0.47 & $\mathrm{~g}[5]$ & -0.69 & -0.83 & 0.70 & -0.27 \\
as[6] & 3.20 & 0.46 & 0.00 & 0.31 & $\mathrm{~g}[6]$ & 0.14 & -0.72 & -0.99 & -0.17 \\
as[7] & 1.56 & -0.90 & -0.57 & -0.05 & $\mathrm{~g}[7]$ & 0.19 & -1.73 & -2.21 & -0.48 \\
as[8] & 1.54 & -0.79 & 0.04 & 0.56 & $\mathrm{~g}[8]$ & 0.64 & -1.75 & 0.96 & 0.47 \\
as[9] & -0.47 & 1.09 & -1.05 & 1.16 & $\mathrm{~g}[9]$ & -3.06 & -0.17 & 1.52 & -0.46 \\
as[10] & 1.46 & 1.23 & 0.70 & -0.87 & $\mathrm{~g}[10]$ & 2.22 & -1.69 & -0.97 & -0.12 \\
as[11] & -0.89 & -0.64 & 0.72 & -1.30 & $\mathrm{~g}[11]$ & -1.59 & -1.68 & 0.12 & 1.08 \\
as[12] & 0.27 & 0.08 & 0.09 & -0.85 & $\mathrm{~g}[12]$ & -0.07 & -1.48 & 0.20 & 0.82 \\
as[13] & 0.26 & -1.11 & -1.18 & 0.80 & $\mathrm{~g}[13]$ & 0.13 & -0.50 & -0.39 & 0.40 \\
as[14] & 2.96 & -2.78 & -0.27 & 0.03 & $\mathrm{~g}[14]$ & 1.62 & -1.15 & -1.26 & 0.11 \\
as[15] & 2.65 & -1.74 & -0.19 & 1.71 & $\mathrm{~g}[15]$ & 0.02 & 0.20 & 0.48 & 2.01 \\
\hline
\end{tabular}

Table C.6. Gelman and Rubin Statistics for Anti-slip and Guessing Parameters

\begin{tabular}{cccccc}
\hline Parameter & Est. & $\begin{array}{c}\text { Upper } \\
\text { CI }\end{array}$ & Parameter & Est. & $\begin{array}{c}\text { Upper } \\
\text { CI }\end{array}$ \\
\hline as[1] & 1.00 & 1.01 & $\mathrm{~g}[1]$ & 1.00 & 1.00 \\
as[2] & 1.00 & 1.00 & $\mathrm{~g}[2]$ & 1.00 & 1.00 \\
as[3] & 1.00 & 1.00 & $\mathrm{~g}[3]$ & 1.00 & 1.00 \\
$\operatorname{as}[4]$ & 1.00 & 1.00 & $\mathrm{~g}[4]$ & 1.00 & 1.00 \\
$\operatorname{as}[5]$ & 1.00 & 1.00 & $\mathrm{~g}[5]$ & 1.00 & 1.00 \\
$\operatorname{as}[6]$ & 1.00 & 1.00 & $\mathrm{~g}[6]$ & 1.00 & 1.00 \\
$\operatorname{as}[7]$ & 1.00 & 1.00 & $\mathrm{~g}[7]$ & 1.00 & 1.00 \\
$\operatorname{as}[8]$ & 1.00 & 1.00 & $\mathrm{~g}[8]$ & 1.00 & 1.01 \\
$\operatorname{as}[9]$ & 1.00 & 1.00 & $\mathrm{~g}[9]$ & 1.00 & 1.01 \\
$\operatorname{as}[10]$ & 1.00 & 1.00 & $\mathrm{~g}[10]$ & 1.00 & 1.00 \\
$\operatorname{as}[11]$ & 1.00 & 1.00 & $\mathrm{~g}[11]$ & 1.00 & 1.00 \\
$\operatorname{as}[12]$ & 1.00 & 1.01 & $\mathrm{~g}[12]$ & 1.00 & 1.00 \\
$\operatorname{as}[13]$ & 1.00 & 1.00 & $\mathrm{~g}[13]$ & 1.00 & 1.00 \\
$\operatorname{as}[14]$ & 1.00 & 1.00 & $\mathrm{~g}[14]$ & 1.00 & 1.00 \\
$\operatorname{as}[15]$ & 1.00 & 1.00 & $\mathrm{~g}[15]$ & 1.00 & 1.00 \\
\hline
\end{tabular}




\section{DINA with Saturated structure}

Table C.7. Geweke Statistics for Anti-slip and Guessing Parameters

\begin{tabular}{cccccccccc}
\hline Parameter & Chain1 & Chain2 & Chain3 & Chain4 & Parameter & Chain1 & Chain2 & Chain3 & Chain4 \\
\hline as[1] & -2.26 & 0.02 & -1.34 & -0.04 & $\mathrm{~g}[1]$ & 0.71 & -0.88 & -1.52 & -0.32 \\
as[2] & 1.54 & 0.91 & -0.10 & 0.06 & $\mathrm{~g}[2]$ & 0.21 & -0.40 & 0.23 & 0.08 \\
as[3] & -1.24 & -1.92 & -1.66 & 0.28 & $\mathrm{~g}[3]$ & -3.11 & 1.04 & -1.10 & -0.31 \\
as[4] & -0.01 & 0.75 & 0.36 & 1.49 & $\mathrm{~g}[4]$ & 1.08 & -0.42 & -0.26 & -0.95 \\
as[5] & -1.74 & 1.72 & 0.91 & 1.24 & $\mathrm{~g}[5]$ & 1.89 & -1.86 & -2.52 & 0.27 \\
as[6] & 0.88 & 1.16 & -2.14 & 0.62 & $\mathrm{~g}[6]$ & -2.71 & -1.18 & 0.55 & -0.79 \\
as[7] & -2.03 & -0.99 & 1.41 & 2.14 & $\mathrm{~g}[7]$ & -0.48 & 1.01 & -0.36 & 0.41 \\
as[8] & 0.79 & -1.16 & -0.94 & 0.78 & $\mathrm{~g}[8]$ & -2.13 & 1.01 & -0.78 & 0.68 \\
as[9] & 0.78 & -0.43 & -0.86 & 0.75 & $\mathrm{~g}[9]$ & -0.81 & 1.68 & -0.98 & 0.51 \\
as[10] & 2.34 & -0.81 & 0.02 & 1.86 & $\mathrm{~g}[10]$ & -1.87 & 0.07 & 1.37 & -0.16 \\
as[11] & 0.72 & 1.44 & 0.65 & 1.41 & $\mathrm{~g}[11]$ & -1.70 & -0.07 & -2.80 & 0.33 \\
as[12] & -0.70 & -1.77 & 0.25 & -1.80 & $\mathrm{~g}[12]$ & 0.27 & -0.43 & -0.12 & -1.79 \\
as[13] & -1.21 & 0.11 & 0.05 & 0.20 & $\mathrm{~g}[13]$ & -0.05 & 1.28 & 1.16 & 0.69 \\
as[14] & -1.46 & 0.62 & -0.16 & -0.45 & $\mathrm{~g}[14]$ & 1.58 & 0.45 & 0.63 & -0.65 \\
as[15] & 0.04 & -0.33 & -0.90 & 1.17 & $\mathrm{~g}[15]$ & 1.34 & -0.08 & 0.68 & 1.57 \\
\hline
\end{tabular}

Table C.8. Gelman and Rubin Statistics for Anti-slip and Guessing Parameters

\begin{tabular}{cccccc}
\hline Parameter & Est. & $\begin{array}{c}\text { Upper } \\
\text { CI }\end{array}$ & Parameter & Est. & $\begin{array}{c}\text { Upper } \\
\text { CI }\end{array}$ \\
\hline as[1] & 1.00 & 1.00 & $\mathrm{~g}[1]$ & 1.00 & 1.00 \\
$\operatorname{as}[2]$ & 1.00 & 1.00 & $\mathrm{~g}[2]$ & 1.00 & 1.00 \\
$\operatorname{as}[3]$ & 1.00 & 1.00 & $\mathrm{~g}[3]$ & 1.00 & 1.01 \\
$\operatorname{as}[4]$ & 1.00 & 1.01 & $\mathrm{~g}[4]$ & 1.00 & 1.00 \\
$\operatorname{as}[5]$ & 1.00 & 1.00 & $\mathrm{~g}[5]$ & 1.00 & 1.00 \\
$\operatorname{as}[6]$ & 1.00 & 1.00 & $\mathrm{~g}[6]$ & 1.00 & 1.00 \\
$\operatorname{as}[7]$ & 1.00 & 1.00 & $\mathrm{~g}[7]$ & 1.00 & 1.00 \\
$\operatorname{as}[8]$ & 1.00 & 1.00 & $\mathrm{~g}[8]$ & 1.00 & 1.00 \\
$\operatorname{as}[9]$ & 1.00 & 1.00 & $\mathrm{~g}[9]$ & 1.00 & 1.01 \\
$\operatorname{as}[10]$ & 1.00 & 1.00 & $\mathrm{~g}[10]$ & 1.00 & 1.00 \\
$\operatorname{as}[11]$ & 1.00 & 1.00 & $\mathrm{~g}[11]$ & 1.00 & 1.00 \\
$\operatorname{as}[12]$ & 1.00 & 1.00 & $\mathrm{~g}[12]$ & 1.00 & 1.00 \\
$\operatorname{as}[13]$ & 1.00 & 1.00 & $\mathrm{~g}[13]$ & 1.00 & 1.00 \\
$\operatorname{as}[14]$ & 1.00 & 1.00 & $\mathrm{~g}[14]$ & 1.00 & 1.00 \\
$\operatorname{as}[15]$ & 1.00 & 1.00 & $\mathrm{~g}[15]$ & 1.00 & 1.00 \\
\hline
\end{tabular}




\section{GDM with Constant Slopes}

Table C.9. Geweke Statistics for Intercept and Slope Parameters

\begin{tabular}{|c|c|c|c|c|c|c|c|c|c|}
\hline $\begin{array}{c}\text { Paramete } \\
\text { r }\end{array}$ & $\begin{array}{c}\text { Chain } \\
1\end{array}$ & $\begin{array}{c}\text { Chain } \\
2\end{array}$ & $\begin{array}{c}\text { Chain } \\
3\end{array}$ & $\begin{array}{c}\text { Chain } \\
4\end{array}$ & Parameter & $\begin{array}{c}\text { Chain } \\
1\end{array}$ & $\begin{array}{c}\text { Chain } \\
2\end{array}$ & $\begin{array}{c}\text { Chain } \\
3\end{array}$ & $\begin{array}{c}\text { Chain } \\
4\end{array}$ \\
\hline beta[1] & -0.98 & -1.80 & -0.76 & 0.48 & gamma[1] & 1.15 & 1.17 & 0.35 & -0.39 \\
\hline beta[2] & -3.02 & -1.77 & 0.45 & -0.24 & gamma[2] & 2.48 & 2.21 & -1.10 & -0.40 \\
\hline beta[3] & -0.04 & -2.83 & -1.09 & 0.60 & gamma[3] & -1.85 & 2.20 & 0.06 & 0.67 \\
\hline beta[4] & -2.54 & 0.68 & 0.54 & 1.35 & gamma[4] & 2.24 & -0.84 & -0.78 & -1.47 \\
\hline beta[5] & -2.23 & -1.18 & -1.38 & -0.60 & gamma[5] & -1.28 & -1.98 & -1.82 & -0.20 \\
\hline beta[6] & -1.11 & 0.09 & -1.56 & -0.63 & gamma[6] & 1.11 & -0.15 & 1.41 & 0.50 \\
\hline beta[7] & -2.23 & -0.53 & -2.02 & -0.71 & gamma[7] & 2.33 & 0.52 & 1.87 & 0.55 \\
\hline beta[8] & -1.07 & 0.31 & -1.66 & 0.87 & gamma[8] & 1.18 & -0.22 & 1.69 & -0.93 \\
\hline beta[9] & 1.54 & 0.06 & 1.16 & -0.66 & gamma[9] & -2.66 & -1.57 & -2.81 & 0.62 \\
\hline beta[10] & 0.11 & 1.01 & 2.06 & 1.61 & gamma[10] & -0.86 & -2.37 & -2.90 & -1.56 \\
\hline beta[11] & -1.10 & 0.01 & -1.91 & -1.12 & gamma[11] & -1.34 & -1.54 & -0.97 & 1.00 \\
\hline beta[12] & -0.98 & 0.27 & -0.99 & 0.77 & gamma[12] & -0.14 & -0.99 & -1.33 & -0.82 \\
\hline beta[13] & -2.91 & -0.34 & -1.46 & -0.41 & gamma[13] & 2.97 & 0.27 & 1.18 & 0.27 \\
\hline beta[14] & 1.35 & -2.00 & -1.22 & -0.96 & gamma[14] & -1.51 & 1.89 & 1.22 & 1.05 \\
\hline beta[15] & -2.20 & -0.80 & -2.41 & -0.87 & gamma[15] & 2.19 & 0.81 & 2.51 & 0.84 \\
\hline
\end{tabular}

Table C.10. Gelman and Rubin Statistics for Intercept and Slope Parameters

\begin{tabular}{cccccc}
\hline Parameter & Est. & $\begin{array}{c}\text { Upper } \\
\text { CI }\end{array}$ & Parameter & Est. & $\begin{array}{c}\text { Upper } \\
\text { CI }\end{array}$ \\
\hline beta[1] & 1.02 & 1.05 & gamma[1] & 1.02 & 1.05 \\
beta[2] & 1.00 & 1.01 & gamma[2] & 1.00 & 1.01 \\
beta[3] & 1.00 & 1.00 & gamma[3] & 1.00 & 1.00 \\
beta[4] & 1.02 & 1.06 & gamma[4] & 1.02 & 1.06 \\
beta[5] & 1.00 & 1.01 & gamma[5] & 1.11 & 1.16 \\
beta[6] & 1.01 & 1.02 & gamma[6] & 1.01 & 1.02 \\
beta[7] & 1.01 & 1.03 & gamma[7] & 1.01 & 1.03 \\
beta[8] & 1.08 & 1.16 & gamma[8] & 1.08 & 1.14 \\
beta[9] & 1.00 & 1.01 & gamma[9] & 1.01 & 1.02 \\
beta[10] & 1.03 & 1.08 & gamma[10] & 1.01 & 1.03 \\
beta[11] & 1.00 & 1.01 & gamma[11] & 1.02 & 1.05 \\
beta[12] & 1.05 & 1.10 & gamma[12] & 1.08 & 1.13 \\
beta[13] & 1.01 & 1.03 & gamma[13] & 1.01 & 1.03 \\
beta[14] & 1.00 & 1.01 & gamma[14] & 1.00 & 1.01 \\
beta[15] & 1.01 & 1.01 & gamma[15] & 1.01 & 1.01 \\
\hline
\end{tabular}




\section{GDM with Varying Slopes}

Table C.11. Geweke Statistics for Intercept and Slope Parameters

\begin{tabular}{|c|c|c|c|c|c|c|c|c|c|}
\hline & Chain1 & Chain2 & Chain3 & Chain4 & Parameter & Chain1 & Chain2 & Chain3 & Chain4 \\
\hline beta[1] & 1.49 & -0.18 & -0.90 & 1.63 & $\operatorname{gamma}[1,1]$ & -1.48 & 0.14 & 0.99 & -1.64 \\
\hline beta[2] & 0.05 & 0.33 & 0.79 & 1.71 & $\operatorname{gamma}[2,1]$ & -0.34 & -0.06 & -2.66 & 3.76 \\
\hline beta[3] & 0.67 & -0.77 & 0.55 & 0.98 & gamma[2,2] & 0.98 & -1.55 & 0.99 & 0.35 \\
\hline $\operatorname{beta}[4]$ & 1.01 & -0.24 & 1.19 & 2.61 & $\operatorname{gamma}[2,3]$ & 0.96 & 0.74 & -1.03 & -3.17 \\
\hline beta[5] & -0.16 & 0.77 & 0.40 & 0.16 & $\operatorname{gamma}[2,4]$ & 0.74 & -0.18 & 0.58 & -1.02 \\
\hline beta[6] & 0.29 & -0.66 & 1.78 & 4.51 & $\operatorname{gamma}[3,1]$ & 0.98 & 0.34 & 0.16 & 0.99 \\
\hline beta[7] & 1.11 & 0.07 & 0.36 & 0.13 & $\operatorname{gamma}[4,1]$ & -0.50 & 1.51 & -1.49 & -0.04 \\
\hline beta[8] & -1.76 & 1.28 & 1.53 & -0.69 & $\operatorname{gamma}[4,2]$ & 0.01 & -0.65 & 1.21 & 1.28 \\
\hline beta[9] & -0.12 & 0.26 & 2.69 & 0.09 & $\operatorname{gamma}[4,3]$ & 0.26 & -0.57 & -0.74 & -1.54 \\
\hline beta[10] & 2.15 & -1.14 & 0.64 & 1.90 & $\operatorname{gamma}[4,4]$ & -1.33 & 0.65 & -0.68 & -2.19 \\
\hline beta[11] & -0.58 & 0.65 & 2.08 & 0.80 & $\operatorname{gamma}[4,5]$ & -1.31 & -1.84 & 0.02 & -3.50 \\
\hline beta[12] & -0.52 & -0.82 & 0.40 & -0.76 & $\operatorname{gamma}[5,3]$ & -0.56 & -1.03 & -0.86 & 0.36 \\
\hline beta[13] & 0.63 & 2.04 & 1.83 & 1.14 & $\operatorname{gamma}[6,1]$ & 2.92 & 1.28 & -0.84 & 1.71 \\
\hline beta[14] & 1.23 & 0.39 & -0.63 & 1.64 & $\operatorname{gamma}[6,2]$ & -2.92 & -0.20 & -0.97 & -3.57 \\
\hline beta[15] & 0.46 & -2.01 & 2.68 & 1.29 & $\operatorname{gamma}[6,3]$ & 1.82 & 0.12 & -0.03 & 0.00 \\
\hline & & & & & $\operatorname{gamma}[6,4]$ & 0.75 & -0.03 & 0.69 & -3.27 \\
\hline & & & & & $\operatorname{gamma}[7,1]$ & 2.89 & 0.90 & -2.36 & 2.01 \\
\hline & & & & & $\operatorname{gamma}[7,2]$ & -5.39 & -0.61 & 0.94 & -0.14 \\
\hline & & & & & $\operatorname{gamma}[7,3]$ & 3.73 & -0.12 & -0.54 & 1.07 \\
\hline & & & & & $\operatorname{gamma}[7,4]$ & -0.36 & -0.20 & 1.21 & -0.20 \\
\hline & & & & & $\operatorname{gamma}[8,1]$ & 2.48 & 0.01 & 2.09 & 1.71 \\
\hline & & & & & gamma[8,2] & 1.66 & -1.41 & -1.82 & 1.29 \\
\hline & & & & & $\operatorname{gamma}[9,1]$ & 0.55 & 1.34 & -0.15 & 0.11 \\
\hline & & & & & $\operatorname{gamma}[9,3]$ & -0.06 & -0.53 & -2.31 & 0.73 \\
\hline & & & & & $\operatorname{gamma}[10,1]$ & -1.06 & 2.12 & -1.83 & 0.62 \\
\hline & & & & & $\operatorname{gamma}[10,3]$ & -0.01 & -0.95 & 1.16 & -1.83 \\
\hline & & & & & $\operatorname{gamma}[10,4]$ & -2.11 & 0.90 & 0.57 & -1.75 \\
\hline & & & & & $\operatorname{gamma}[10,5]$ & 0.21 & 0.80 & 0.37 & 0.51 \\
\hline & & & & & $\operatorname{gamma}[11,1]$ & 1.48 & -1.06 & 1.85 & -0.30 \\
\hline & & & & & $\operatorname{gamma}[11,3]$ & 0.29 & -0.40 & -2.32 & -0.81 \\
\hline & & & & & $\operatorname{gamma}[12,1]$ & -1.02 & 0.93 & -2.08 & -0.47 \\
\hline & & & & & gamma[12,3] & 0.75 & 0.60 & -0.11 & 0.96 \\
\hline & & & & & $\operatorname{gamma}[12,4]$ & -2.57 & 1.42 & 0.06 & 0.40 \\
\hline & & & & & $\operatorname{gamma}[13,1]$ & 3.41 & 0.29 & 1.06 & 1.22 \\
\hline & & & & & $\operatorname{gamma}[13,2]$ & -2.81 & -0.74 & -2.19 & -3.91 \\
\hline & & & & & $\operatorname{gamma}[13,3]$ & 1.08 & -1.02 & -0.49 & 0.05 \\
\hline & & & & & $\operatorname{gamma}[13,4]$ & 0.04 & -0.72 & -0.30 & -1.84 \\
\hline & & & & & $\operatorname{gamma}[14,1]$ & -0.72 & 0.80 & 0.73 & 1.76 \\
\hline & & & & & $\operatorname{gamma}[14,2]$ & -3.22 & -0.40 & 0.75 & -2.04 \\
\hline & & & & & $\operatorname{gamma}[14,3]$ & 2.25 & -0.03 & -0.94 & -0.59 \\
\hline & & & & & $\operatorname{gamma}[14,4]$ & -0.69 & -0.28 & 0.91 & -3.49 \\
\hline & & & & & $\operatorname{gamma}[14,5]$ & -2.30 & -1.91 & 0.06 & -1.59 \\
\hline & & & & & $\operatorname{gamma}[15,1]$ & 1.44 & 0.96 & 1.57 & 4.20 \\
\hline & & & & & $\operatorname{gamma}[15,2]$ & -2.35 & 0.45 & -0.63 & -1.62 \\
\hline & & & & & $\operatorname{gamma}[15,3]$ & 2.87 & 0.07 & -1.87 & 1.53 \\
\hline & & & & & gamma $[15,4]$ & 0.19 & 1.04 & -2.89 & -0.89 \\
\hline
\end{tabular}


Table C.12. Gelman and Rubin Statistics for Intercept Parameters

\begin{tabular}{|c|c|c|c|c|c|}
\hline Parameter & Est. & Upper CI & Parameter & Est. & Upper CI \\
\hline beta[1] & 1.02 & 1.05 & gamma[1,1] & 1.02 & 1.05 \\
\hline beta[2] & 1.00 & 1.01 & gamma[2,1] & 1.00 & 1.01 \\
\hline beta[3] & 1.00 & 1.00 & gamma[2,2] & 1.01 & 1.02 \\
\hline beta[4] & 1.02 & 1.04 & gamma[2,3] & 1.00 & 1.01 \\
\hline beta[5] & 1.00 & 1.01 & gamma[2,4] & 1.00 & 1.01 \\
\hline beta[6] & 1.01 & 1.04 & $\operatorname{gamma}[3,1]$ & 1.00 & 1.00 \\
\hline beta[7] & 1.01 & 1.03 & gamma[4,1] & 1.01 & 1.03 \\
\hline beta[8] & 1.01 & 1.02 & $\operatorname{gamma}[4,2]$ & 1.01 & 1.02 \\
\hline beta[9] & 1.00 & 1.01 & $\operatorname{gamma}[4,3]$ & 1.00 & 1.00 \\
\hline beta[10] & 1.01 & 1.02 & $\operatorname{gamma}[4,4]$ & 1.02 & 1.06 \\
\hline beta[11] & 1.00 & 1.01 & $\operatorname{gamma}[4,5]$ & 1.00 & 1.01 \\
\hline beta[12] & 1.04 & 1.09 & gamma[5,3] & 1.00 & 1.01 \\
\hline beta[13] & 1.05 & 1.14 & gamma[6,1] & 1.01 & 1.03 \\
\hline beta[14] & 1.01 & 1.02 & gamma[6,2] & 1.03 & 1.10 \\
\hline \multirow[t]{33}{*}{ beta[15] } & 1.01 & 1.03 & gamma[6,3] & 1.01 & 1.04 \\
\hline & & & gamma[6,4] & 1.00 & 1.01 \\
\hline & & & gamma[7,1] & 1.08 & 1.21 \\
\hline & & & gamma[7,2] & 1.03 & 1.10 \\
\hline & & & gamma[7,3] & 1.02 & 1.06 \\
\hline & & & gamma[7,4] & 1.00 & 1.00 \\
\hline & & & $\operatorname{gamma}[8,1]$ & 1.26 & 1.73 \\
\hline & & & gamma[8,2] & 1.07 & 1.18 \\
\hline & & & $\operatorname{gamma}[9,1]$ & 1.00 & 1.01 \\
\hline & & & gamma[9,3] & 1.00 & 1.01 \\
\hline & & & $\operatorname{gamma}[10,1]$ & 1.01 & 1.02 \\
\hline & & & $\operatorname{gamma}[10,3]$ & 1.00 & 1.01 \\
\hline & & & gamma[10,4] & 1.01 & 1.02 \\
\hline & & & gamma[10,5] & 1.00 & 1.01 \\
\hline & & & $\operatorname{gamma}[11,1]$ & 1.00 & 1.01 \\
\hline & & & $\operatorname{gamma}[11,3]$ & 1.01 & 1.02 \\
\hline & & & gamma[12,1] & 1.00 & 1.01 \\
\hline & & & gamma[12,3] & 1.03 & 1.07 \\
\hline & & & gamma[12,4] & 1.01 & 1.02 \\
\hline & & & $\operatorname{gamma}[13,1]$ & 1.02 & 1.06 \\
\hline & & & gamma[13,2] & 1.09 & 1.26 \\
\hline & & & gamma[13,3] & 1.02 & 1.03 \\
\hline & & & $\operatorname{gamma}[13,4]$ & 1.00 & 1.02 \\
\hline & & & $\operatorname{gamma}[14,1]$ & 1.01 & 1.01 \\
\hline & & & $\operatorname{gamma}[14,2]$ & 1.05 & 1.14 \\
\hline & & & gamma[14,3] & 1.04 & 1.11 \\
\hline & & & gamma[14,4] & 1.02 & 1.04 \\
\hline & & & gamma[14,5] & 1.01 & 1.02 \\
\hline & & & $\operatorname{gamma}[15,1]$ & 1.05 & 1.13 \\
\hline & & & gamma[15,2] & 1.07 & 1.20 \\
\hline & & & $\operatorname{gamma}[15,3]$ & 1.04 & 1.10 \\
\hline & & & $\operatorname{gamma}[15,4]$ & 1.02 & 1.05 \\
\hline & & & $\operatorname{gamma}[15,4]$ & 1.02 & 1.05 \\
\hline
\end{tabular}

